\title{
WestVirginiaUniversity
}

THE RESEARCH REPOSITORY @ WVU

Graduate Theses, Dissertations, and Problem Reports

2002

\section{Small cycle cover, group coloring with related problems}

\author{
Xiangwen Li \\ West Virginia University
}

Follow this and additional works at: https://researchrepository.wvu.edu/etd

\section{Recommended Citation}

Li, Xiangwen, "Small cycle cover, group coloring with related problems" (2002). Graduate Theses,

Dissertations, and Problem Reports. 1600.

https://researchrepository.wvu.edu/etd/1600

This Dissertation is protected by copyright and/or related rights. It has been brought to you by the The Research Repository @ WVU with permission from the rights-holder(s). You are free to use this Dissertation in any way that is permitted by the copyright and related rights legislation that applies to your use. For other uses you must obtain permission from the rights-holder(s) directly, unless additional rights are indicated by a Creative Commons license in the record and/ or on the work itself. This Dissertation has been accepted for inclusion in WVU Graduate Theses, Dissertations, and Problem Reports collection by an authorized administrator of The Research Repository @ WVU.

For more information, please contact researchrepository@mail.wvu.edu. 


\title{
Small Cycle Cover, Group Coloring With Related Problems
}

\author{
Xiangwen Li \\ Dissertation Submitted to the \\ Eberly College of Arts and Sciences \\ at West Virginia University \\ in partial fulfillment of the requirements \\ for the degree of \\ Doctor of Philosophy \\ in \\ Mathematics \\ Hong-Jian Lai Ph.D., Chair \\ Zhi-Hong Chen Ph.D \\ Elaine M Eschen Ph.D. \\ John Goldwasser Ph.D. \\ Jerzy Wojcienchowski Ph.D. \\ Cun-Qian Zhang Ph.D. \\ Morgantown, West Virginia \\ 2002
}

Keywords: List Coloring, Group Chromatic number, Line Graph

Copyright 2002 Xiangwen Li 


\title{
ABSTRACT \\ Small Cycle Cover, Group Coloring With Related Problems
}

\author{
Xiangwen Li
}

Bondy conjectured that if $G$ is a simple 2-connected graph with $n \geq 3$ vertices, then the edges of $G$ can be covered by at most $\frac{2 n-3}{3}$ cycles. In Chapter 2, a result on small cycle cover is obtained and we also show that the result is as best as possible.

Thomassen conjectured that every 4-connected line graph is hamiltonian. In Chapters 3 and 4, we apply Catlin's reduction method to study cycles in line graphs. Results about hamiltonian connectivity of line graphs and 3-edge-connected graphs are obtained. Several former results are extended.

Jaeger, Linial, Payan and Tarsi introduced group coloring in 1992 and proved that the group chromatic number for every planar graph is at most 6 . It is shown that the bound 6 can be decreased to 5. Jaeger, Linial, Payan and Tarsi also proved that the group chromatic number for every planar graph with girth at least 4 is at most 4 . Chapters 5 and 6 are devoted to the study of group coloring of graphs.

The concept of list coloring, choosability and choice number was introduced by Erdos, Rubin and Taylor in 1979 and Vizing in 1976. Alon and Tarsi proved that every bipartite planar graph is 3-choosable. Thomassen showed that every planar graph is 5-choosable and that every planar graph with girth at least 5 is 3 -choosable. In Chapter 7 , results on list coloring are obtained, extending a former result of Thomassen. 


\section{Acknowledgements}

I would like to thank my supervisor, Dr. Hong-Jian Lai, for his guidance, advice, and continual encouragement. It is a pleasure to work under his supervision. Without him, this dissertation could not have come out.

I would also like to thank other committee members: Dr. Zhi-Hong Chen, Dr. J. Goldwasser, Dr. W. Wojcienchowski, Dr. E. M. Eschen, and Dr. C-Q Zhang, for their help during my studies.

And finally, I thank my family: my wife Jinshun, for her never ending support; my mother Mrs. Fan, for her understanding; and my daughter Sha and my son Richard, for helping me keep a good perspective on life. 


\title{
Dedication
}

\author{
To \\ my mother \\ and \\ to the memory of
}

my father 


\section{Contents}

1 Introduction 1

1.1 Definitions and Notations .............. 1

1.2 Results ..................... . . . 6

2 Small Cycle Cover of 2-Connected Cubic Graphs 14

2.1 Introduction . . . . . . . . . . . . . . . . . . 14

2.2 Lemmas . . . . . . . . . . . . . . . . . . 16

2.3 Proof of Theorem 2.1.1 . . . . . . . . . . . . . . . . . 19

3 Hamiltonian Connected Line Graphs 29

3.1 Introduction . . . . . . . . . . . . . . . . . 29

3.2 Catlin's Reduction Method . . . . . . . . . . . . . 34

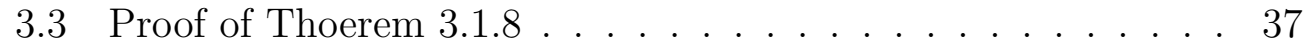

3.4 Generalizations and Applications ............ 40

4 Circuits containing 12 vertices in 3 -edge-connected graphs and Hamiltonian line graphs $\quad 44$

4.1 Introduction . . . . . . . . . . . . . . . . 44

4.2 Catlin's Reduction Method . . . . . . . . . . . . . . . . 49 
4.3 Proof of Theorem $4.1 .9 \ldots \ldots$. . . . . . . . . . . 51

4.4 Proofs for Theorems 4.1 .2 and $4.1 .3 \ldots . . . . . . .556$

5 Group chromatic number of some graphs $\quad 60$

5.1 Introduction . . . . . . . . . . . . . . 60

5.2 The group chromatic number of $K_{3,3}$-minor free graphs . . . . 61

5.3 The group chromatic number for join of two graphs . . . . . . 63

5.4 The group chromatic number for the $k^{\text {th }}$ power of a graph . . 65

6 Group chromatic number of planar graphs with girth at least $\begin{array}{ll}4 & 68\end{array}$

6.1 Introduction . . . . . . . . . . . . . . 68

$6.2 \quad Z_{3}$-Coloring Theorem ............... . . 71

$6.3 Z_{3}$-coloring of $K_{3,3}$-minor free graphs . . . . . . . . . . . . . 91

7 3-list coloring of planar graphs with girth at least $4 \quad 95$

7.1 Introduction . . . . . . . . . . . . . . 95

7.2 A list coloring Theorem . . . . . . . . . . . . . 96

7.3 3-list coloring of $K_{3,3}$-minor free graphs . . . . . . . . . . 111 


\section{Chapter 1}

\section{Introduction}

\subsection{Definitions and Notations}

All graphs in this dissertation are finite and undirected without loops. A graph $G$ is an ordered pair $(V(G), E(G))$ consisting of a nonempty set $V(G)$ of vertices, a set $E(G)$, disjoint from $V(G)$, of edges. Two vertices $u, v$ are adjacent if $u v \in E(G)$. A graph is simple if it has no loops and no two of its edges join the same pair of vertices. A connected graph with at least two vertices is called a nontrivial graph.

For a set $S,|S|$ denotes the cardinality of $S$. For a graph $G,|V(G)|$ is often denoted by $n$ and we shall assume that $n \geq 1$.

For a vertex $u \in V(G)$, we denote the neghborhood of $u$ of $G$ by $N(u)=$ $\{v: u v \in E(G)\}$. The degree $d_{G}(v)$ of a vertex $v$ in $G$ is the number of edges of $G$ incident with $v$, each loop counting as two edges. We define

$$
\Delta(G)=\max _{v \in V(G)} d(v), \delta(G)=\min _{v \in V(G)} d(v)
$$


Let $G$ be a graph. If $G$ is connected and the degree of every vertex is even, then $G$ is an Eulerian graph. An Eulerian subgraph of $G$ is called a circuit. A trail of $G$ is defined as a vertex-edge alternating sequence

$$
v_{0}, e_{1}, v_{1}, e_{2}, \cdots, e_{k}, v_{k}
$$

such that all the $e_{i}$ 's are distinct and such that for each $i=1,2, \cdots, k, e_{i}$ is incident with both $v_{i-1}$ and $v_{i}$.

If $x$ is a real number, then the ceiling of $x$, denoted $\lceil x\rceil$, is the smallest integer which is not less than $x$. If $U$ is a subset of $V(G)$, then $G[U]$ denote the subgraph of $G$ induced by $U$. If $E_{1}$ is a subset of $E(G)$, then $G\left[E_{1}\right]$ denote the subgraph of $G$ induced by $E_{1}$.

A $k$-path $(k$-cycle $)$ denotes a path (cycle) with $k$ edges. The distance of 4-cycle $v_{1} v_{2} v_{3} v_{4} v_{1}$ and 5-cycle $u_{1} u_{2} u_{3} u_{4} u_{5} u_{1}$ is $\min \left\{d_{G}\left(v_{i}, u_{j}\right) \mid 1 \leq i \leq 4,1 \leq\right.$ $j \leq 5\}$, where $d_{G}(u, v)$ denotes the length of a shortest $(u, v)$-path in $G$. The girth of graph $G$ is the length of a shortest cycle of $G$. For a plane graph the unique unbounded face is called the outer face. If $C$ is a cycle in a plane graph, then $\operatorname{int}(C)$ is the set of vertices and edges inside $C$. If $\operatorname{int}(\mathrm{C})=\emptyset$, then $C$ is facial. If the outer face is bounded by a cycle, we call it the outer cycle. A separating cycle is a cycle $C$ in a plane graph such that the graph has at least one vertex outside $C$ and at least one vertex inside $C$. Let $G$ be a graph. A set of subgraphs of $G$ is said to be independent if no two of them have common vertex.

A cycle cover of a graph $G$ is a collection $\mathcal{C}$ of cycles of $G$, such that every edge of $G$ lies in at least one member of $\mathcal{C}$. A cycle double cover of $G$ is a cycle cover $\mathcal{C}$ of $G$ such that each edge of $G$ lies in exactly two members of $C$. 
Let $G$ and $H$ be two graphs. $G$ is contractible to $H$ if $H$ can be obtained from $G$ by contracting some edges of $G$ and deleting the resulting loops. If $G$ contains a subgraph which is contractible to $H$, then $H$ is a minor of $G$.

The edge-connectivity of a graph $G$ is denoted by $\kappa^{\prime}(G)$. We use $H \subseteq G$ ( $H \subset G$ ) to denote the fact that $H$ is a subgraph of $G$ (proper subgraph of $G$ ). If $H \subset G$, then for an edge subset $X \subseteq E(G)-E(H)$, we write $H+X$ for $G[E(H) \cup X]$. When $X=\{e\}$, we also use $H+e$ for $H+\{e\}$.

Let $X \subseteq E(G)$. The contraction $G / X$ is obtained from $G$ by contracting each edge of $X$ and deleting the resulting loops. If $H \subseteq G$, we write $G / H$ for $G / E(H)$.

A subgraph $\Gamma$ of $G$ is called an $R$-subgraph if both $O(\Gamma)=R$ and $G-E(\Gamma)$ is connected. A graph $G$ is collapsible if for any even subset $R$ of $V(G), G$ has an $R$-subgraph. The reduction of $G$ is obtained from $G$ by contracting all maximal collapsible subgraph. A graph $G$ is reduced if $G$ has no nontrivial collapsible subgraphs.

If $H$ is a connected subgraph of $G$, and if $v_{H}$ denotes the vertex in $G / H$ to which $H$ is contracted, then $H$ is called the preimage of $v_{H}$. A vertex $v$ in a contraction of $G$ is nontrivial if $v$ has a nontrivial preimage.

Let $O(G)$ denote the set of odd degree vertices of $G$. A graph $G$ is Eulerian if $O(G)=\emptyset$ and $G$ is connected. A graph $G$ is supereulerian if $G$ has a spanning Eulerian subgraph. In particular, $K_{1}$ is both Eulerian and supereulerian.

A subgraph $H$ of a graph $G$ is dominating if $G-V(H)$ is edgeless. A dominating eulerian subgraph is also called a DES. For an integer $i \geq 1$, 
define

$$
D_{i}(G)=\{v \in V(G): d(v)=i\} .
$$

The line graph of a graph $G$, denoted by $L(G)$, has $E(G)$ as its vertex set, where two vertices in $L(G)$ are adjacent if and only if the corresponding edges in $G$ are adjacent.

Let $A$ denote an Abelian group and $F(G, A)$ denote the set of all functions from $E(G)$ to $A$. For $f \in F(G, A)$, an $(A, f)$-coloring of $G$ under the orientation $D$ is a function $c: V(G) \mapsto A$ such that for every directed edge $e=u v$ oriented from $u$ to $v$, it always has $c(u)-c(v) \neq f(u v)$. $G$ is $A$ colorable under the orientation $D$ if for any function $f \in F(G, A)$, G has an $(A, f)$-coloring. It is known $([34])$ that $A$-colorablity is independent of the choice of the orientation. The group chromatic number of a graph $G$ is defined to be the smallest positive integer $m$ for which $G$ is $A$-colorable for any Abelian group $A$ of order $\geq m$ under a given orientation $D$, and is denoted by $\chi_{g}(G)$.

Let $G$ be a directed graph. For $u, v \in V(G),(u, v)$ denotes a directed edge oriented from $u$ to $v$. For a vertex $v \in V(G)$, let

$$
E_{G}^{-}(v)=\{(u, v) \in E(G): u \in V(G)\} \text { and } E_{G}^{+}(v)=\{(v, u) \in E(G): u \in
$$
$V(G)\}$.

Let $E(v)=E^{+}(v) \cup E^{-}(v)$ and let $f: E \mapsto Z$, where $Z$ is the set of integer and let

$$
f^{+}(v)=\sum_{e \in E^{+}(v)} f(e) \text { and } f^{-}(v)=\sum_{e \in E^{-}(v)} f(e) .
$$

A flow of $G$ is a function $f$ such that $f^{+}(v)=f^{-}(v)$ for every vertex $v \in$ 
$V(G)$. If there exists such a function such that $0<|f(e)|<k$ for every edge $e \in E(G)$, we call $G$ admits a nowhere-zero $k$-flow.

Let $A$ be a nontrivial additive Abelian group, let 0 denote the identity element of $A$ and let $A^{*}=A-\{0\}$. Define

$$
F(G, A)=\{f: E(G) \mapsto A\} \text { and } F^{*}(G, A)=\left\{f: E(G) \mapsto A^{*}\right\}
$$

For each $f \in F(G, A)$, the boundary of $f$ is a function $\partial f: V(G) \mapsto A$ defined by

$$
\partial f(v)=\sum_{e \in E^{+}(v)} f(e)-\sum_{e \in E^{-}(v)} f(e),
$$

where " $\sum$ " refers to the addition in $A$.

Let $G$ be an undirected graph and $A$ be an Abelian group. Denote

$$
Z(G, A)=\left\{b: V(G) \mapsto A \text { such that } \sum_{v \in V(G)} b(v)=0\right\} .
$$

A graph $G$ is $A$-connected if $G$ has an orientation $G^{\prime}$ such that for every function $b \in Z(G, A)$ there is a function $f \in F^{*}\left(G^{\prime}, A\right)$ such that $b=\partial f$. For an Abelian group $A$, let $\langle A\rangle$ denote the family of graphs that are $A$ connected. It is well-known that $G \in<A>$ is independent of the orientation of $G$.

Let $k$ be a positive integer and let $G^{k}$ denote the $k$ th power of $G$ : i.e., the graph with $V\left(G^{k}\right)=V(G)$, where $u v \in E\left(G^{k}\right)$ if and only if $u$ and $v$ lie at distance at most $k$ in $G$.

Let $G$ be a graph and let $L(v)$ be a set of allowed colors for each vertex $v$. An L-list coloring of a graph $G$ is a proper vertex coloring in which every vertex $v$ gets a color from $L(v)$. $G$ is $k$-choosable if $G$ has a list coloring for each list assignment with $k$ colors in each list. 


\section{$1.2 \quad$ Results}

In this dissertation, we study three problems: cycle cover, hamiltionian line graph, and coloring.

The cycle double cover conjecture ([46], [45]) states that every bridgeless graph has a cycle double cover. It is known that if the cycle double cover conjecture is false, then a minimal counterexample would be a 2-edge-connected simple cubic graph. Bondy [7] conjectured that every simple 2-connected cubic graph on $n$ vertices admits a cycle double cover $\mathcal{C}$ with $|\mathcal{C}| \leq \frac{n}{2}$.

Bondy [7] also conjectured that if $G$ is a simple 2-connected graph with $n \geq 3$ vertices, then the edges of $G$ can be covered by at most $\frac{2 n-3}{3}$ cycles. Recently, Fan [23] proved this conjecture. Barnette [4] proved that if $G$ is a 3-connected planar graph of order $n$, then the edges of $G$ can be covered by at most $\frac{n+1}{2}$ cycles. In Chapter 2 , the following is proved.

Theorem 1.2.1 Every 2-connected simple cubic graph of order $n \geq 6$ admits a cycle cover $\mathcal{C}$ with $|\mathcal{C}| \leq\left\lceil\frac{n}{4}\right\rceil$.

The result of Theorem 1.2.1 is sharp in the sense that there exists an infinite family of 2-connected cubic graphs with $|V(G)| \geq 6$ such that each graph $G$ in the family requires at least $\left\lceil\frac{|V(G)|}{4}\right\rceil$ cycles in any cycle cover of $G$.

Harary and Nash-Williams gave a close relationship between dominating eulerian subgraphs in a graph $G$ and Hamilton cycles in $L(G)$.

Theorem 1.2.2 (Harary and Nash-Williams [29]) Let $G$ be a graph with $|E(G)| \geq 3$. Then $L(G)$ is hamiltonian if and only if $G$ has a dominating 
eulerian subgraph.

Thomassen [47] conjectured that every 4-connected line graph is hamiltonian. The best result in this direction is that every 7-connected line graph is hamiltonian connected ([57].

An edge $e \in E(G)$ is called subdivided when it is replaced by a path of length 2 whose internal vertex, denoted $v(e)$, has degree 2 in the resulting graph. The process of taking an edge $e$ and replacing it by that length 2 path is called subdividing e. For a graph $G$ and edges $e^{\prime}, e^{\prime \prime} \in E(G)$, let $G\left(e^{\prime}\right)$ denote the graph obtained from $G$ by subdividing $e^{\prime}$, and let $G\left(e^{\prime}, e^{\prime \prime}\right)$ denote the graph obtained from $G$ by subdividing both $e^{\prime}$ and $e^{\prime \prime}$.

In Chapter 3, we prove the following.

Theorem 1.2.3 Suppose that $G$ satisfies the following conditions (F1) and (F2):

(F1) If $X$ is an edge cut of $G$ with $|X| \leq 3$, then there exists a vertex $v \in D_{|X|}(G)$ such that $X$ consists of all the edges incident with $v$ in $G$, and

(F2) for every $v \in D_{3}(G)$, $v$ lies in a $k$-cycle $C_{v}$ of $G$, where $2 \leq k \leq 3$. If $\kappa^{\prime}(G) \geq 3$, then for every pair of edges $e^{\prime}, e^{\prime \prime} \in E(G)$ we have

(i) $G\left(e^{\prime}, e^{\prime \prime}\right)$ is collapsible and

(ii) G has a spanning $\left(v\left(e^{\prime}\right), v\left(e^{\prime \prime}\right)\right)$-trail.

This theorem has a number of corollaries.

Corollary 1.2.4 Let $G$ be a graph such that the set of neighbors of each vertex of degree 3 in $G$ is not an independent set. If $L(G)$ is 4-connected, $L(G)$ is hamiltonian connected. 
Corollary 1.2.5 (Kriesell, [37]) If $G$ is a $K_{1,3}$-free graph and if $L(G)$ is 4-connected, then $L(G)$ is hamiltonian connected.

Corollary 1.2.6 (Zhan, [57]) If $\kappa^{\prime}(G) \geq 4$, then $L(G)$ is hamiltonian connected.

Corollary 1.2.7 If $L^{2}(G)$ is 4-connected, then $L^{2}(G)$ is hamiltonian connected.

Let $C_{4}$ denote a 4-cycle in $K_{5}$. The graph $K_{5}-E\left(C_{4}\right)$ is called an hourglass. A graph $G$ is hourglass free if $G$ does not have an induced subgraph isomorphic to $K_{5}-E\left(C_{4}\right)$.

Corollary 1.2.8 (Broersma, Kriesell and Ryjacek, [9]) Every 4-connected hourglass free line graph is hamiltonian connected.

In Chapter 4, we study the existence of a circuit $H$ in a 3-edge-connected graph $G$ such that $H$ contains a given set of vertices of $G$. We prove the following:

Theorem 1.2.9 Let $G$ be a 3-edge-connected graph and let $S \subseteq V(G)$ be a vertex subset such that $|S| \leq 12$. Then either $G$ has a circuit $H$ such that $S \subseteq V(H)$, or $G$ can be contracted to the Petersen graph in such a way that the preimage of each vertex of the Petersen graph contains at least one vertex in $S$.

If $G$ is a planar graph, we have 
Theorem 1.2.10 Let $G$ be a 3-edge-connected planar graph, and let $S \subseteq$ $V(G)$ be a vertex subset such that $|S| \leq 23$. Then there is a circuit in $G$ containing $S$.

When $G$ is a cubic graph, $\kappa(G)=\kappa^{\prime}(G)$ and every circuit $H$ of $G$ is a cycle of $G$. Therefore, Theorem 1.2.9 and Theorem 1.2.10 extend the following results in [5] and [1], respectively.

Theorem 1.2.11 (Bau and Holton [5]). Let $G$ be a 3-connected cubic graph and let $S \subseteq V(G)$ be a vertex subset such that $|S| \leq 12$. Then either $G$ has a cycle $H$ such that $S \subseteq V(H)$, or $G$ is contractible to the Petersen graph in such a way that the preimage of each vertex of the Petersen graph contains at least one vertex in $S$.

Theorem 1.2.12 (Aldred, Bau, Holton and McKay [1]). If $S$ is a set of at most 23 vertices in a 3-connected cubic planar graph $G$, then there is a cycle in $G$ containing $S$.

There are infinite examples which show that the requirement of 12 vertices in Theorem 1.2.9 and Theorem 1.2.11 cannot be replaced by 13 vertices. The sharpness of Theorem 1.2 and Theorem 1.2.10 is demonstrated in [31] that there are 3-edge-connected (cubic) planar graphs in which there is a set of 24 vertices that do not lie on a common cycle.

Jaeger et al [34] proved the following result.

Theorem 1.2.13 (Jeager, Linial, Payan, and Tarsi [34]). If $G$ is a simple planar graph, then $\chi_{g}(G) \leq 6$. 
This has been improved by Lai and Zhang.

Theorem 1.2.14 ([39]) If $G$ is a simple graph without a $K_{5}$-minor, then $\chi_{g}(G) \leq 5$. In particular, if $G$ is a simple planar graph, then $\chi_{g}(G) \leq 5$.

Let $H \subset G$ be graphs, and $A$ be a group. Given an $f \in F(G, A)$, if for an $\left(A,\left.f\right|_{E(H)}\right)$-coloring $c_{0}$ of $H$, there is an $(A, f)$-coloring $c$ of $G$ such that $c$ is an extension of $c_{0}$, then we say that $c_{0}$ is extended to $c$. If any $\left(A,\left.f\right|_{E(H)}\right)$-colotring $c_{0}$ of $H$ can be extended to an $(A, f)$-coloring $c$, then we say that $(G, H)$ is $(A, f)$-extendsible. If for any $f \in F(G, A),(G, H)$ is $(A, f)$-extensible, then $(G, H)$ is $A$-extensible.

In Chapter 5, we will prove the following result which extends also Jeager et al [34] result.

Theorem 1.2.15 Let $G$ be a connected simple graph without a $K_{3,3}$ minor and let $A$ be a group with $|A| \geq 5$. Suppose that $H$ is a subgraph of $G$ isomorphic to $K_{2}$. Then $(G, H)$ is A-extensible.

Let $G_{1}$ and $G_{2}$ be subgraphs of $G$. The union $G_{1} \cup G_{2}$ of $G_{1}$ and $G_{2}$ is the subgraph with vertex set $V\left(G_{1}\right) \cup V\left(G_{2}\right)$ and edge set $E\left(G_{1}\right) \cup E\left(G_{2}\right)$. If $G_{1}$ and $G_{2}$ are disjoint, denote the union by $G_{1}+G_{2}$. The join $G \vee H$ of disjoint graphs $G$ and $H$ is the graph obtained from $G+H$ by joining each vertex of $G$ to each vertex of $H$. Let $G$ and $H$ be two given graphs. In proper coloring, it is well known that $\chi(G \vee H)=\chi(G)+\chi(H)$, but it is different for group coloring. It is easy to see that $\chi\left(K_{2,2}\right)=2$ and $K_{2,2}=\overline{K_{2}} \vee \overline{K_{2}}$. Lai and Zhang [38] showed that $\chi_{g}\left(K_{2,2}\right)=3$. In Chapter 5, we prove the following result. 
Theorem 1.2.16 (1) Suppose that $G$ and $H$ are disjoint graphs. Then

$$
\chi_{g}(G+H)=\max \left\{\chi_{g}(G), \chi_{g}(H)\right\} .
$$

(2) Let $H_{1}$ and $H_{2}$ be subgraphs of $G$ such that $V\left(H_{1}\right) \cap V\left(H_{2}\right)=\emptyset$ and $V(G)=V\left(H_{1}\right) \cup V\left(H_{2}\right)$. Then

$$
\chi_{g}(G) \leq \min \left\{\max \left\{\chi_{g}\left(H_{1}\right), \max _{v \in V\left(H_{2}\right)} \operatorname{deg}\left(v, H_{1}\right)\right\}+\Delta\left(H_{2}\right)+1,\right.
$$

$\left.\max \left\{\chi_{g}\left(H_{2}\right), \max _{u \in V\left(H_{1}\right)} \operatorname{deg}\left(u, H_{2}\right)\right\}+\Delta\left(H_{1}\right)+1\right\}$.

The bound of Theorem 5.4.2 is sharp. From Theorem 5.4.2, we obtain the following corollary.

Corollary 1.2.17 Suppose that $G$ and $H$ are disjoint graphs. Then

$$
\chi_{g}(G \vee H) \leq \min \left\{\left|V\left(H_{1}\right)\right|+\Delta\left(H_{2}\right)+1,\left|V\left(H_{2}\right)\right|+\Delta\left(H_{1}\right)+1\right\} .
$$

Lai and Zhang [38] proved the group coloring analogue of Brook's Theorem [10].

Theorem 1.2.18 (Lai and Zhang [38]) For any connected simple graph $G$,

$$
\chi_{g}(G) \leq \Delta(G)+1,
$$

with equality if and if either $\Delta(G)=2$ and $G$ is a cycle; or $\Delta(G) \geq 3$ and $G$ is complete.

In Chapter 5, we obtain the following result which extends Theorem 5.4.1.

Theorem 1.2.19 Let $k$ be an integer. If $G$ is a connected graph, then

$$
\chi_{g}\left(G^{k}\right) \leq \Delta^{k}+1,
$$

and equality holds if and only if either $G=K_{2}$ or $G$ is a $\Delta$-regular graph of girth $2 k+1$ and order $\Delta^{k}+1$. 
Jaeger, Linial, Payan and Tarsi [34] proved that if $G$ is a planar graph, then $\chi_{g}(G) \leq 6$. It is shown (see [38]) that if $G$ is a graph without a $K_{5^{-}}$ minor or without a $K_{3,3}$-minor, then $\chi_{g}(G) \leq 5$. Jaeger, Linial, Payan and Tarsi [34] also proved that if $G$ is a planar graph with girth at least 4, then $\chi_{g}(G) \leq 4$. In Chapter 6, we prove the following results.

Theorem 1.2.20 Suppose that $G$ is a planar graph with girth 4 such that all 4-cycles are independent and every 4-cycle is facial. If the minimum distance between 4 -cycles and 5 -cycles is at least 1 , then $\chi_{g}(G) \leq 3$.

Theorem 1.2.21 If $G$ is a $K_{3,3}$-minor free graph with girth at least 5 , then $\chi_{g}(G) \leq 3$.

Jaeger, Linial, Payan and Tarsi [34] had the following conjecture.

Conjecture 1.2.22 Every 5-edge connected graph is $Z_{3}$-connected.

Let $G$ be a connected plane graph, $G^{*}$ the geometric dual of $G$, and $A$ an Abelian group. Jeager et al [34] showed that $G$ is $A$-connected if and only if $G^{*}$ is $A$-colorable. By Theorem 1.2.20, we comfirm this conjecture for planar graphs.

Corollary 1.2.23 Every 5-edge connected planar graph is $Z_{3}$-connected.

The concept of $L$-list coloring, choosability and choice number was introduced by Erdos, Rubin and Taylor [22] in 1979 and Vizing [53] in 1976.

Alon and Tarsi [2] proved that every bipartite planar graph is 3-choosable. Thomassen [48] showed that every planar graph is 5-choosable. Voigt [54] presented an example of a planar graph which is not 4-choosable. 
Theorem 1.2.24 (Thomassen [49]) Every planar graph of girth at least 5 is 3-choosable.

In Chapter 7, we use a similar technique to prove the following extensions of Theorem 7.1.1.

Theorem 1.2.25 Suppose that $G$ is a planar graph with girth at least 4 such that all 4-cycles are independent and every 4-cycle is facial. If the minimum distance between 4-cycles and 5-cycles is at least 1, then $G$ is 3-choosable.

Theorem 1.2.26 If $G$ is a connected $K_{3,3}$-minor free graph with girth at least 5, then $G$ is 3-choosable.

The conditions of Theorem 7.1.2 can not be relax in the sense that Gutner's example [26] with only 164 vertices is not 3-choosable but there exists a 5-cycle adjacent to a 4-cycle in his graph. 


\section{Chapter 2}

\section{Small Cycle Cover of 2-Connected Cubic Graphs}

\section{$2.1 \quad$ Introduction}

A cycle cover of a graph $G$ is a collection $\mathcal{C}$ of cycles of $G$ such that every edge of $G$ lies in at least one member of $\mathcal{C}$. A cycle double cover of $G$ is a cycle cover $\mathcal{C}$ of $G$ such that each edge of $G$ lies in exactly two members of $\mathcal{C}$. The cycle double conjecture ([46], [45], and [58]) states that every bridgeless graph has a cycle double cover. It is known that if the cycle double cover conjecture is false, then a minimal counterexample would be a 2-edge-connected simple cubic graph. Bondy [7] conjectured that every 2-connected simple cubic graph on $n$ vertices admits a double cycle cover $\mathcal{C}$ with $|\mathcal{C}| \leq \frac{n}{2}$.

Bondy [7] also conjectured that if $G$ is a 2-connected simple graph with 
$n \geq 3$ vertices, then the edges of $G$ can be covered by at most $\frac{2 n-3}{3}$ cycles. Earlier, Y. X. Luo and R. S. Chen [41] proved that this conjecture holds for 2-connected simple cubic graphs. Recently Fan [23] settled this conjecture by showing that it holds for all simple 2-connected graphs. Barnette [4] proved that if $G$ is a 3-connected simple planar graph of order $n$, then the edges of $G$ can be covered by at most $\frac{n+1}{2}$ cycles. In this chapter, the following is proved.

Theorem 2.1.1 Every 2-connected simple cubic graph of order $n \geq 6$ admits a cycle cover $\mathcal{C}$ with $|\mathcal{C}| \leq\left\lceil\frac{n}{4}\right\rceil$.

The result of Theorem 2.1.1 is sharp in the sense that there exists an infinite family of 2-connected cubic graphs such that each graph $G$ in the family requires at least $\left\lceil\frac{|V(G)|}{4}\right\rceil$ cycles in any cycle cover of $G$ (see Figure 1).

Let $L_{1}, L_{2}$ be two graphs isomorphic to $K_{4}-e$, i.e. $K_{4}$ minus an edge. Let $G$ denote the graph in Figure 1. Note that $n=|V(G)|=2 \mu+8$. Since a cycle of $G$ contains at most 2 edges in $\left\{e_{1}, e_{2}, \ldots, e_{\mu}\right\}$, and since it takes at least 2 cycles to cover $E\left(L_{1}\right) \cup E\left(L_{2}\right)$, it follows that any cycle cover of $G$ must have at least $\left\lceil\frac{\mu}{2}\right\rceil+2=\left\lceil\frac{n}{4}\right\rceil$ cycles.

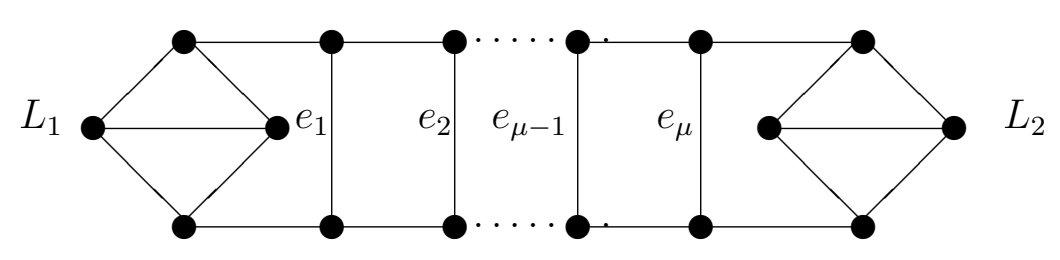

Figure 2.1 


\section{$2.2 \quad$ Lemmas}

Let $G$ be a 2-connected simple cubic graph and let $T=\left\{u_{1}, u_{2}, u_{3}\right\}$ be a 3 -cut of $G$. If there is no vertex $v \in V(G)$ such that $N(v)=T, T$ is called a nontrivial 3-cut. Similarly we can define nontrivial 3-edge cut of G. A set of subgraphs of $G$ is said to be independent if no two of them have a common vertex.

The removal of an edge $e=u v$ in a cubic graph $G$ with $N(u)=\left\{x_{1}, y_{1}, v\right\}$ and $N(v)=\left\{x_{2}, y_{2}, v\right\}$ is to remove $e$ and to replace the path $x_{1} u y_{1}$ and $x_{2} v y_{2}$ by the edges $x_{1} y_{1}$ and $x_{2} y_{2}$ respectively. Denote by $(G-e)^{*}$ the resulting graph. Note that when $G$ is a simple graph, $(G-e)^{*}$ may be not a simple graph.

The following lemma is straightforward.

Lemma 2.2.1 Let $G$ be a simple cubic graph. Then

(i) $G$ is $k$-edge-connected if and only if $G$ is $k$-connected, where $1 \leq k \leq 3$.

(ii) Suppose that $G$ is a 3-edge-connected graph of order $n \geq 6$. Then $G$ does not contain two distinct triangles $T$ and $T^{\prime}$ such that $E(T) \cap E\left(T^{\prime}\right) \neq \emptyset$.

Lemma 2.2.2 Let $G$ be a 3-connected simple cubic graph of order $n>4$ and let $T=x_{1} x_{2} x_{3} x_{1}$ be a triangle of $G$ and let $y_{i}$ be the neighbor of $x_{i}$ for $1 \leq i \leq 3$ such that $\left\{y_{1}, y_{2}, y_{3}\right\} \cap V(T)=\emptyset$. Suppose that $T$ is contracted into one vertex $x$ and that $G^{\prime}$ is the resulting graph. If $G^{\prime}$ has a cycle cover $\mathcal{C}^{\prime}$, then $G$ has a cycle cover $\mathcal{C}$ such that $|\mathcal{C}|=\left|\mathcal{C}^{\prime}\right|$.

Proof. By Lemma 2.2.1, all triangles of $G$ must be independent. Since $G$ is 3-connected and cubic, it follows that $G^{\prime}$ is a 3-connected cubic graph with 
$\left|V\left(G^{\prime}\right)\right|=|V(G)|-2$. Since $d_{G^{\prime}}(x)=3, \mathcal{C}^{\prime}$ must have at least two cycles $C_{1}^{*}, C_{2}^{*}$ of $\mathcal{C}^{\prime}$ which pass all the three edges incident with $x$.

Assume that $C_{1}, C_{2}, \ldots C_{s}$ are all the cycles in $\mathcal{C}^{\prime}$ containing the edges $y_{1} x, y_{2} x$, that $D_{1}, D_{2}, \ldots, D_{t}$ are all the cycles in $\mathcal{C}^{\prime}$ containing the edges $y_{1} x, y_{3} x$, and that $Z_{1}, \ldots, Z_{l}$ are all the cycles in $\mathcal{C}^{\prime}$ containing the edges $y_{2} x, y_{3} x$. Without loss of generality, we assume that $s \geq 1$ and $t \geq 1$. Extend the cycles of $G^{\prime}$ to the cycles of $G$ as follows:

$$
\begin{aligned}
C_{i}^{*}= & C_{i}-\left\{x y_{1}, x y_{2}\right\} \cup\left\{x_{1} y_{1}, x_{1} x_{3}, x_{2} x_{3}, y_{2} x_{2}\right\}, i=1,2, \cdots, s, \\
D_{j}^{*}= & D_{j}-\left\{x y_{1}, x y_{3}\right\} \cup\left\{x_{1} y_{1}, x_{1} x_{2}, x_{2} x_{3}, y_{3} x_{3}\right\}, j=1,2, \cdots, t, \\
Z_{k}^{*}= & Z_{k}-\left\{x y_{2}, x y_{3}\right\} \cup\left\{x_{2} y_{2}, x_{2} x_{3}, y_{3} x_{3}\right\}, k=1,2, \cdots, l, \\
\mathcal{C}= & \mathcal{C}^{\prime}-\left(\left\{C_{i}: 1 \leq i \leq s\right\} \cup\left\{D_{j}: 1 \leq j \leq t\right\} \cup\left\{Z_{k}: 1 \leq k \leq l\right\}\right) \\
& \cup\left\{C_{i}^{*}: 1 \leq i \leq s\right\} \cup\left\{D_{j}^{*}: 1 \leq j \leq t\right\} \cup\left\{Z_{k}^{*}: 1 \leq k \leq l\right\} .
\end{aligned}
$$

Then $\mathcal{C}$ is a cycle cover of $G$ with $|\mathcal{C}|=\left|\mathcal{C}^{\prime}\right|$.

A graph $G$ is essentially 4-edge-connected if it is 3-edge-connected and, if $G-S$ is disconnected for some set $S$ of three edges of $G$, then $G-S$ has exactly two components, one of which is a single vertex. It follows that if $G$ is a 3-connected cubic graph and $G$ does not contain any nontrivial 3-edge cuts, then $G$ is essentially 4 -edge-connected.

Lemma 2.2.3 Let $G$ be a triangle free simple cubic graph. Then

(i) Suppose that $G$ is a 3-edge-connected graph of order $n \geq 8$. Then there is an edge e such that $(G-e)^{*}$ is a 2-connected simple cubic graph.

(ii) Suppose that $G$ is an essentially 4-edge-connected graph of order $n \geq$ 12. Then $G$ has two distinct edges $e_{1}, e_{2}$ such that $\left(\left(G-e_{1}\right)^{*}-e_{2}\right)^{*}$ is a 2-connected simple cubic graph. 
Proof. (1) Let $e=u v \in E(G)$ and $N(u)=\left\{z_{1}, z_{2}, v\right\}$ and $N(v)=$ $\left\{w_{1}, w_{2}, u\right\}$. Since $G$ is triangle free, $z_{1} z_{2} \notin E(G), w_{1} w_{2} \notin E(G)$ and $\left\{z_{1}, z_{2}\right\} \cap$ $\left\{w_{1}, w_{2}\right\}=\emptyset$. Since $G$ is 3-edge-conneced, $(G-e)^{*}$ is a connected simple cubic graph. Assume that $e^{\prime}$ is an edge cut of $(G-e)^{*}$. Then $\left\{e^{\prime}, e\right\}$ must contains an edge cut of $G$, contrary to the assumption that $\kappa^{\prime}(G) \geq 3$.

(2) Since $n \geq 12$, we can choose two distinct edges $e_{1}=u_{1} v_{1}, e_{2}=u_{2} v_{2}$ with

$N\left(u_{1}\right)=\left\{x_{1}, y_{1}, v_{1}\right\}, N\left(v_{1}\right)=\left\{x_{2}, y_{2}, u_{1}\right\}, N\left(u_{2}\right)=\left\{s_{1}, t_{1}, v_{2}\right\}, N\left(v_{2}\right)=\left\{s_{2}, t_{2}, u_{2}\right\}$

such that

$$
\left\{x_{1}, y_{1}, x_{2}, y_{2}, u_{1}, v_{1}\right\} \cap\left\{s_{1}, t_{1}, s_{2}, t_{2}, u_{2}, v_{2}\right\}=\emptyset .
$$

Since $G$ is triangle free, we have

$$
\left\{x_{1} y_{1}, x_{2} y_{2}, s_{1} t_{1}, s_{2} t_{2}\right\} \cap E(G)=\emptyset
$$

and

$$
\left|\left\{x_{1}, x_{2}, y_{1}, y_{2}\right\}\right|=\left|\left\{s_{1}, t_{1}, s_{2}, t_{2}\right\}\right|=4 \text {. }
$$

Thus $\left(\left(G-e_{1}\right)^{*}-e_{2}\right)^{*}$ is a simple cubic graph. Since $G$ is essentially 4-edge-connected, $\left(\left(G-e_{1}\right)^{*}-e_{2}\right)^{*}$ is connected. Assume that $e$ is an edge cut of $\left(\left(G-e_{1}\right)^{*}-e_{2}\right)^{*}$. Then $\left(\left(G-e_{1}\right)^{*}\right)-e_{2}^{*}-e$ has only two components. Since $\left(\left(G-e_{1}\right)^{*}-e_{2}\right)^{*}$ is cubic, each component of $\left(\left(G-e_{1}\right)^{*}-e_{2}\right)^{*}$ has at least 2 vertices. Therefore $\left\{e_{1}, e_{2}, e\right\}$ must contain an edge cut of $G$, contrary to that $G$ is essentially 4 -edge-connected. Therefore $\left(\left(G-e_{1}\right)^{*}-e_{2}\right)^{*}$ is a 2-connected simple cubic graph. 


\subsection{Proof of Theorem 2.1.1}

We argue by induction on $n=|V(G)|$. As $G$ is cubic, $|V(G)|$ is even. When $n=6, G$ is one of the two graphs in Figure 2. It is easy to verify that each of $G_{1}$ and $G_{2}$ has a cycle cover $\mathcal{C}$ with $|\mathcal{C}|=2$.
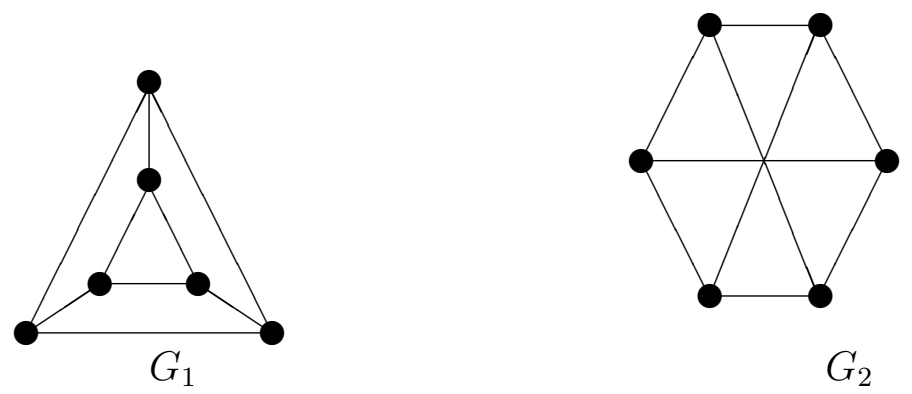

Figure 2.2: $G_{1}$ and $G_{2}$

Now we assume that $n \geq 8$. Consider these cases.

Case $1 G$ has a 2-cut $\{u, v\}$ such that $u v \in E(G)$.

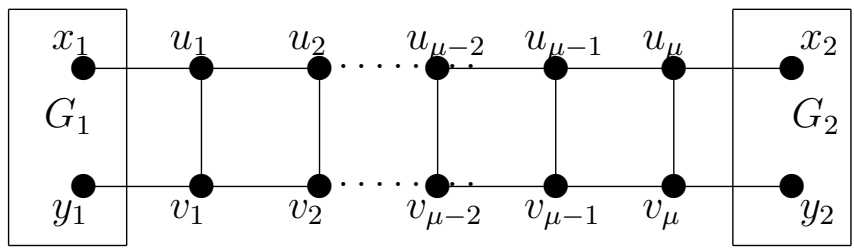

Figure 2.3: $G_{3}$ in the Proof for Case 1 
Then $G$ has two vertex disjoint subgraphs $G_{1}$ and $G_{2}$ with nonadjacent vertices $x_{i}, y_{i} \in V\left(G_{i}\right),(1 \leq i \leq 2)$, and a ladder $G_{3}$ whose ends are $x_{1}, y_{1}, x_{2}$ and $y_{2}$, such that $E(G)=E\left(G_{1}\right) \cup E\left(G_{2}\right) \cup E\left(G_{3}\right)$, as shown in Figure 3 .

Let $u_{1}, v_{1}, u_{\mu}, v_{\mu} \in V\left(G_{3}\right)$ with $x_{1} u_{1}, y_{1} v_{1}, x_{2} u_{\mu}, y_{2} v_{\mu} \in E(G)$. We define the three graphs $G_{1}^{*}, G_{2}^{*}$ and $G_{3}^{*}$ from $G$ as follows: $G_{i}^{*}=G_{i}+x_{i} y_{i}, i=1,2$ and $G_{3}^{*}=G-V\left(G_{1} \cup G_{2}\right)$. Let $\left|V\left(G_{i}^{*}\right)\right|=n_{i}, i=1,2,3$. It follows that $n_{1}+n_{2}+n_{3}=n$, that both $G_{1}^{*}$ and $G_{2}^{*}$ are two 2-connected simple cubic graphs and that both $n_{1}$ and $n_{2}$ are even. By induction, for each $i=1,2$, if $G_{i}^{*} ¥ K_{4}$, then $G_{i}^{*}$ has a cycle cover $\mathcal{C}_{i}$ with $\left|\mathcal{C}_{i}\right| \leq\left\lceil\frac{n_{i}}{4}\right\rceil$. We will distinguish the following subcases.

Subcase $1.1 G_{i}^{*} \nsucceq K_{4}, i=1,2$.

Suppose that $C_{1}, C_{2}, \ldots, C_{s}$ are all the cycles in $\mathcal{C}_{1}$ containing the edge $x_{1} y_{1}$, and $D_{1}, D_{2}, \ldots, D_{t}$ are all the cycles in $\mathcal{C}_{2}$ containing the edge $x_{2} y_{2}$. Note that both $n_{1}$ and $n_{2}$ are even.

Assume first that $\mu=1$. Define

$$
\begin{aligned}
& C_{i}^{*}=C_{i}-x_{1} y_{1} \cup\left\{x_{1} u_{1}, u_{1} v_{1}, v_{1} y_{1}\right\}, i=1,2, \ldots, s, \\
& D_{j}^{*}=D_{j}-x_{2} y_{2} \cup\left\{x_{2} u_{1}, u_{1} v_{1}, v_{1} y_{2}\right\}, j=1,2, \ldots, t .
\end{aligned}
$$

Then

$$
\begin{aligned}
\mathcal{C}= & \left(\mathcal{C}_{1}-\left\{C_{i}: 1 \leq i \leq s\right\}\right) \cup\left(\mathcal{C}_{2}-\left\{D_{j}: 1 \leq j \leq t\right\}\right) \cup\left\{C_{i}^{*}: 1 \leq i \leq s\right\} \\
& \cup\left\{D_{j}^{*}: 1 \leq j \leq t\right\}
\end{aligned}
$$

is a cycle cover of $G$ with $|\mathcal{C}|=\left|\mathcal{C}_{1}\right|+\left|\mathcal{C}_{2}\right| \leq\left\lceil\frac{n_{1}}{4}\right\rceil+\left\lceil\frac{n_{2}}{4}\right\rceil \leq\left\lceil\frac{n}{4}\right\rceil$. 
Suppose $\mu \geq 2$. For $i=1,2, \ldots, s$ and for $j=1,2, \ldots, t$, define

$$
\begin{aligned}
C_{i}^{*}= & C_{i} \cup D_{1}-\left\{x_{1} y_{1}, x_{2} y_{2}\right\} \\
& \cup\left\{u_{i} u_{i+1}, v_{i} v_{i+1}: 1 \leq i \leq \mu-1\right\} \cup\left\{x_{1} u_{1}, y_{1} v_{1}, x_{2} u_{\mu}, y_{2} v_{\mu}\right\}, \\
D_{j}^{*}= & D_{j} \cup C_{1}-\left\{x_{1} y_{1}, x_{2} y_{2}\right\} \\
& \cup\left\{u_{i} u_{i+1}, v_{i} v_{i+1}: 1 \leq i \leq \mu-1\right\} \cup\left\{x_{1} u_{1}, y_{1} v_{1}, x_{2} u_{\mu}, y_{2} v_{\mu}\right\} .
\end{aligned}
$$

Also, for $i=1,2, \ldots k$, define $Z_{i}=u_{2 i-1} u_{2 i} v_{2 i} v_{2 i-1} u_{2 i-1}$, and when $\mu=2 k+1$, we further define $Z_{k+1}=u_{2 k} u_{2 k+1} v_{2 k+1} v_{2 k} u_{2 k}$. Define

$$
\mathcal{C}= \begin{cases}\left(\mathcal{C}_{1}-\left\{C_{i}: 1 \leq i \leq s\right\}\right) \cup\left(\mathcal{C}_{2}-\left\{D_{j}: 1 \leq j \leq t\right\}\right. & \\ \cup\left\{C_{1}^{*}, C_{2}^{*}, \ldots, C_{s}^{*}, D_{2}^{*}, \ldots, D_{t}^{*}\right\} \cup\left\{Z_{1}, Z_{2}, \ldots, Z_{k}\right\} & \text { if } \mu=2 k \\ \left(\mathcal{C}_{1}-\left\{C_{i}: 1 \leq i \leq s\right\}\right) \cup\left(\mathcal{C}_{2}-\left\{D_{j}: 1 \leq j \leq t\right\}\right. & \\ \cup\left\{C_{1}^{*}, C_{2}^{*}, \ldots, C_{s}^{*}, D_{2}^{*}, \ldots, D_{t}^{*}\right\} \cup\left\{Z_{1}, Z_{2}, \ldots, Z_{k+1}\right\} & \text { if } \mu=2 k+1 .\end{cases}
$$

Then $\mathcal{C}$ is a cycle cover of $G$. If $\mu=2 k$, then $n=n_{1}+n_{2}+4 k$ and

$$
|\mathcal{C}|=\left|\mathcal{C}_{1}\right|+\left|\mathcal{C}_{2}\right|-1+k \leq\left\lceil\frac{n_{1}}{4}\right\rceil+\left\lceil\frac{n_{2}}{4}\right\rceil-1+k \leq\left\lceil\frac{n}{4}\right\rceil .
$$

If $\mu=2 k+1$, then $n=n_{1}+n_{2}+4 k+2$ and

$$
|\mathcal{C}|=\left|\mathcal{C}_{1}\right|+\left|\mathcal{C}_{2}\right|-1+k+1 \leq\left\lceil\frac{n_{1}}{4}\right\rceil+\left\lceil\frac{n_{2}}{4}\right\rceil-1+k+1 \leq\left\lceil\frac{n}{4}\right\rceil .
$$

Subcase 1.2 Exactly one of $G_{1}^{*}$ and $G_{2}^{*}$ is isomorphic to $K_{4}$. We may assume that $G_{1}^{*} \cong K_{4}, G_{2}^{*} ¥ K_{4}$.

Since $G_{1}^{*} \cong K_{4}, E\left(G_{1}^{*}\right)$ has a cycle cover $\mathcal{C}_{1}=\left\{C_{1}, C_{2}\right\}$ such that $x_{1} y_{1} \in$ $E\left(C_{1}\right) \cap E\left(C_{2}\right)$. By induction, $G_{2}^{*}$ has a cycle cover $\mathcal{C}_{2}$ with at most $\left\lceil\frac{n_{2}}{4}\right\rceil$ cycles.

Assume that $D_{1}, D_{2}, \ldots, D_{t}$ are all the cycles of $\mathcal{C}_{2}$ that contain the edge $x_{2} y_{2}$. 
If $\mu=1$, then define

$$
\begin{aligned}
C_{1}^{*} & =\left(C_{1}-x_{1} y_{1}\right) \cup\left\{x_{1} u_{1}, u_{1} v_{1}, v_{1} y_{1}\right\}, \\
C_{2}^{*} & =\left(C_{2}-x_{1} y_{1}\right) \cup\left(D_{1}-x_{2} y_{2}\right) \cup\left\{x_{1} u_{1}, v_{1} y_{1}, x_{2} u_{1}, y_{2} v_{1}\right\}, \\
D_{j}^{*} & =\left(C_{2}-x_{1} y_{1}\right) \cup\left(D_{j}-x_{2} y_{2}\right) \cup\left\{x_{1} u_{1}, v_{1} y_{1}, x_{2} u_{1}, y_{2} v_{1}\right\}, 1 \leq j \leq t .
\end{aligned}
$$

Thus $\mathcal{C}=\mathcal{C}_{1} \cup \mathcal{C}_{2}-\left\{C_{1}, C_{2}, D_{1}, D_{2}, \ldots, D_{t}\right\} \cup\left\{C_{1}^{*}, C_{2}^{*}, D_{2}^{*}, \ldots, D_{t}^{*}\right\}$ is a cycle cover of $G$ with $|\mathcal{C}|=\left|\mathcal{C}_{1}\right|+\left|\mathcal{C}_{2}\right|-1 \leq 2+\left\lceil\frac{n_{2}}{4}\right\rceil-1 \leq\left\lceil\frac{n}{4}\right\rceil$.

If $\mu \geq 2$, then define

$$
\begin{aligned}
C_{1}^{*}= & \left(C_{1}-x_{1} y_{1}\right) \cup\left(D_{1}-x_{2} y_{2}\right) \cup\left\{u_{i} u_{i+1}, v_{i} v_{i+1}: 1 \leq i \leq \mu-1\right\} \\
& \cup\left\{x_{1} u_{1}, y_{1} v_{1}, x_{2} u_{\mu}, y_{2} v_{\mu}\right\}, \\
D_{j}^{*}= & \left(C_{1}-x_{1} y_{1}\right) \cup\left(D_{j}-x_{2} y_{2}\right) \cup\left\{u_{i} u_{i+1}, v_{i} v_{i+1}: 1 \leq i \leq \mu-1\right\} \\
& \cup\left\{x_{1} u_{1}, y_{1} v_{1}, x_{2} u_{\mu}, y_{2} v_{\mu}\right\}, 1 \leq j \leq t, \\
C_{2}^{*}= & \left(C_{2}-x_{1} y_{1}\right) \cup\left\{x_{1} u_{1}, u_{1} v_{1}, v_{1} y_{1}\right\} .
\end{aligned}
$$

For $i=1,2, \ldots, k$, define

$$
Z_{i}= \begin{cases}u_{2 i-1} u_{2 i} v_{2 i} v_{2 i-1} u_{2 i-1} & \text { if } \mu=2 k, \\ u_{2 i} u_{2 i+1} v_{2 i+1} v_{2 i} u_{2 i} & \text { if } \mu=2 k+1\end{cases}
$$

Then let

$$
\begin{aligned}
& \mathcal{C}=\mathcal{C}_{1} \cup \mathcal{C}_{2}-\left(\left\{C_{1}, C_{2}, D_{1}, D_{2}, \ldots, D_{t}\right\} \cup\left\{C_{1}^{*}, C_{2}^{*}, D_{2}^{*}, \ldots, D_{t}^{*}\right\}\right. \\
&\left.\cup\left\{Z_{1}, Z_{2}, \ldots, Z_{k}\right\}\right) .
\end{aligned}
$$

Note that if $\mu=2 k$, then $n=4 k+4+n_{2}$ and

$$
|\mathcal{C}|=\left|\mathcal{C}_{1}\right|+\left|\mathcal{C}_{2}\right|-1+k \leq 2+\left\lceil\frac{n_{2}}{4}\right\rceil-1+k \leq 1+k+\left\lceil\frac{n_{2}}{4}\right\rceil \leq\left\lceil\frac{n}{4}\right\rceil ;
$$

and that if $\mu=2 k+1$, then $n=4 k+2+4+n_{2}$ and

$$
|\mathcal{C}|=\left|\mathcal{C}_{1}\right|+\left|\mathcal{C}_{2}\right|-1+k \leq 2+\left\lceil\frac{n_{2}}{4}\right\rceil-1+k \leq 1+k+\left\lceil\frac{n_{2}}{4}\right\rceil \leq\left\lceil\frac{n}{4}\right\rceil .
$$


Subcase $1.3 G_{i}^{*} \cong K_{4}, i=1,2$.

Since $G$ has a 2 -cut $\{u, v\}$ such that $u v \in E(G)$, we have $\mu \geq 1$ and $n \geq$ 10. Let $\mathcal{C}_{1}=\left\{C_{1}, C_{2}\right\}$ be a cycle cover of $G_{1}^{*}$ such that $x_{1} y_{1} \in E\left(C_{1}\right) \cap E\left(C_{2}\right)$, and let $\mathcal{C}_{2}=\left\{D_{1}, D_{2}\right\}$ be a cycle cover of $G_{2}^{*}$ such that $x_{2} y_{2} \in E\left(D_{1}\right) \cap E\left(D_{2}\right)$. Define

$$
\begin{aligned}
C_{1}^{*}= & \left(C_{1}-x_{1} y_{1}\right) \cup\left(D_{1}-x_{2} y_{2}\right) \cup\left\{u_{i} u_{i+1}, v_{i} v_{i+1}: 1 \leq i \leq \mu-1\right\} \\
& \cup\left\{x_{1} u_{1}, y_{1} v_{1}, x_{2} u_{\mu}, y_{2} v_{\mu}\right\}, \\
C_{2}^{*}= & \left(C_{2}-x_{1} y_{1}\right) \cup\left\{x_{1} u_{1}, u_{1} v_{1}, v_{1} y_{1}\right\}, \\
D_{2}^{*}= & \left(D_{2}-x_{2} y_{2}\right) \cup\left\{x_{2} u_{\mu}, u_{\mu} v_{\mu}, v_{\mu} y_{2}\right\} .
\end{aligned}
$$

For $k \geq 2$, and $1 \leq i \leq k-1$, define $Z_{i}=u_{2 i} v_{2 i} v_{2 i+1} u_{2 i+1} u_{2 i}$, and if $\mu=2 k+1$, define $Z_{k}=u_{2 k} v_{2 k} v_{2 k+1} u_{2 k+1} u_{2 k}$. Then

$$
\mathcal{C}= \begin{cases}\left\{C_{1}^{*}, C_{2}^{*}, D_{2}^{*}\right\} \cup\left\{Z_{1}, Z_{2}, \ldots, Z_{k-1}\right\} & \text { if } \mu=2 k, \\ \left\{C_{1}^{*}, C_{2}^{*}, D_{2}^{*}\right\} \cup\left\{Z_{1}, Z_{2}, \ldots, Z_{k}\right\} & \text { if } \mu=2 k+1 .\end{cases}
$$

is a cycle cover of $G$. Note that if $\mu=2 k$, then $n=4+4+4 k$ and $|\mathcal{C}|=3+k-1=\left\lceil\frac{n}{4}\right\rceil$; and that if $\mu=2 k+1$, then $n=4+4+4 k+2$ and $|\mathcal{C}|=3+k=\left\lceil\frac{n}{4}\right\rceil$.

Case 2. $G$ has a 2-cut $\{u, v\}$ but Case 1 does not occur.

Then $G$ has an edge cut $X$ with $|X|=2$ such that $G-X$ is the disjoint union of two subgraphs $G_{1}$ and $G_{2}$, (see Figure 4). Since Case 1 deos not occur, we must have both $x_{1} y_{1} \notin E(G)$ and $x_{2} y_{2} \notin E(G)$. 


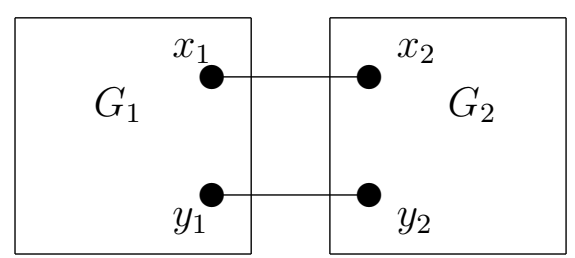

Figure 2.4: The structure of $G$ in Case 2

For $i=1,2$, define $G_{i}^{*}=G_{i}+x_{i} y_{i}$, and let $n_{i}=\left|V\left(G_{i}^{*}\right)\right|$. Note that $n=n_{1}+n_{2}$. By induction, if $G_{i}^{*} \nsucceq K_{4}$, then $G_{i}^{*}$ has a cycle covers $\mathcal{C}_{i}$ with $\left|\mathcal{C}_{i}\right| \leq\left\lceil\frac{n_{i}}{4}\right\rceil$ for $i=1,2$. Since $n \geq 8$, we assume first that $G_{i} \cong K_{4}, i=1,2$. Then $G$ is the graph in Figure 5. It is easy to verify that $G$ has a cycle cover $\mathcal{C}$ with $|\mathcal{C}|=2$.

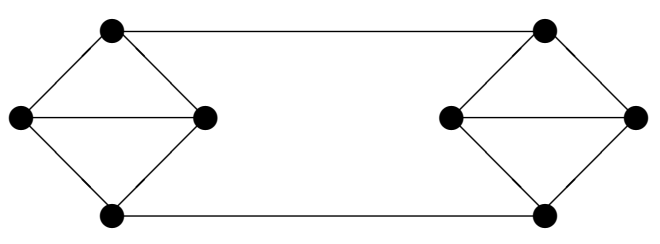

Figure 2.5

We assume then that $G_{1}^{*} \cong K_{4}$ and $G_{2}^{*} \nsubseteq K_{4}$. Thus we may assume that $\mathcal{C}_{1}=\left\{C_{1}, C_{2}\right\}$ such that $x_{1} y_{1} \in E\left(C_{1}\right) \cap E\left(C_{2}\right)$. Let $D_{1}, D_{2}, \ldots, D_{t}$ be all the cycles in $\mathcal{C}_{2}$ containing the edge $x_{2} y_{2}$. Define

$$
\begin{aligned}
& C_{i}^{*}=\left(C_{i}-x_{1} y_{1}\right) \cup\left(D_{1}-x_{2} y_{2}\right) \cup\left\{x_{1} x_{2}, y_{1} y_{2}\right\}, i=1,2 \\
& D_{j}^{*}=\left(C_{1}-x_{1} y_{1}\right) \cup\left(D_{j}-x_{2} y_{2}\right) \cup\left\{x_{1} x_{2}, y_{1} y_{2}\right\}, j=2,3, \ldots, t .
\end{aligned}
$$


Then $\mathcal{C}=\mathcal{C}_{1} \cup \mathcal{C}_{2}-\left\{C_{1}, C_{2}, D_{1}, D_{2}, \ldots, D_{t}\right\} \cup\left\{C_{1}^{*}, C_{2}^{*}, D_{2}^{*}, \ldots, D_{t}^{*}\right\}$ is a cycle cover of $G$. Since $n=n_{2}+4,|\mathcal{C}|=\left|\mathcal{C}_{1}\right|+\left|\mathcal{C}_{2}\right|-1 \leq 2+\left\lceil\frac{n_{2}}{4}\right\rceil-1=\left\lceil\frac{n}{4}\right\rceil$.

Finally we assume that $G_{i}^{*} ¥ K_{4}, i=1,2$. Let $C_{1}, C_{2}, \ldots, C_{s}$ be all the cycles in $\mathcal{C}_{1}$ containing the edge $x_{1} y_{1}$ and let $D_{1}, D_{2}, \ldots, D_{t}$ be all the cycles in $\mathcal{C}_{2}$ containing the edge $x_{2} y_{2}$. Define

$$
\begin{aligned}
& C_{i}^{*}=\left(C_{i}-x_{1} y_{1}\right) \cup\left(D_{1}-x_{2} y_{2}\right) \cup\left\{x_{1} x_{2}, y_{1} y_{2}\right\}, i=1,2, \ldots, s, \\
& D_{j}^{*}=\left(D_{j}-x_{2} y_{2}\right) \cup\left(C_{1}-x_{1} y_{1}\right) \cup\left\{x_{1} x_{2}, y_{1} y_{2}\right\}, j=1,2, \ldots, t .
\end{aligned}
$$

Then $\mathcal{C}=\mathcal{C}_{1} \cup \mathcal{C}_{2}-\left\{C_{1}, C_{2}, \ldots, C_{s}, D_{1}, D_{2}, \ldots, D_{t}\right\} \cup\left\{C_{1}^{*}, C_{2}^{*}, \ldots, C_{s}^{*}, D_{2}^{*}, \ldots, D_{t}^{*}\right\}$ is a cycle cover of $G$. Since $n=n_{1}+n_{2},|\mathcal{C}|=\left|\mathcal{C}_{1}\right|+\left|\mathcal{C}_{2}\right|-1 \leq\left\lceil\frac{n_{1}}{4}\right\rceil+\left\lceil\frac{n_{2}}{4}\right\rceil-1=$ $\left\lceil\frac{n}{4}\right\rceil$.

Case 3. $\kappa(G) \geq 3$.

We first prove the following claim.

Claim Let $G$ be a 3-connected simple cubic graph of order $6<n \leq 10$, then $G$ has a cycle cover $\mathcal{C}$ with $|\mathcal{C}| \leq\left\lceil\frac{n}{4}\right\rceil$.

Proof. Assume that $n=8$. By Lemmas 2.2.1 and 2.2.2, $G$ is triangle free and hence $G$ is one of the two graphs in Figure 6. It is easy to verify that each of which has a cycle cover $\mathcal{C}$ with $|\mathcal{C}|=2$.

So we assume that $n=10$. By Lemmas 2.2.1 and 2.2.2, we assume that $G$ is triangle free. By Lemma 2.2.3 (i), there is an edge $e=x y$ with $N(x)=\left\{x_{1}, y_{1}, y\right\}$ and $N(y)=\left\{x_{2}, y_{2}, x\right\}$ such that $(G-e)^{*}$ is a 2 connected simple cubic graph. By $\left|V\left((G-e)^{*}\right)\right|=8$ and by Case $2,(G-e)^{*}$ has a cycle cover $\mathcal{C}^{\prime}=\left\{D_{1}, D_{2}\right\}$. Extend the cycles of $G^{\prime}$ to cycles of $G$ as follows. 
For each $i \in\{1,2\}$, define

$$
D_{i}^{*}= \begin{cases}D_{i}-x_{1} y_{1} \cup\left\{x_{1} x, x y_{1}\right\} & \text { if } x_{1} y_{1} \in E\left(D_{i}\right), \\ D_{i}-x_{2} y_{2} \cup\left\{x_{2} y, y y_{2}\right\} & \text { if } x_{2} y_{2} \in E\left(D_{i}\right), \\ D_{i} & \text { if }\left\{x_{1} y_{1}, x_{2} y_{2}\right\} \cap E\left(D_{i}\right)=\emptyset .\end{cases}
$$

Since $G$ is 3 -connected, there is a cycle $C$ in $G$ with $x y \in E(C)$, and so $\left\{D_{1}^{*}, D_{2}^{*}, C\right\}$ is a cycle cover of $G$.
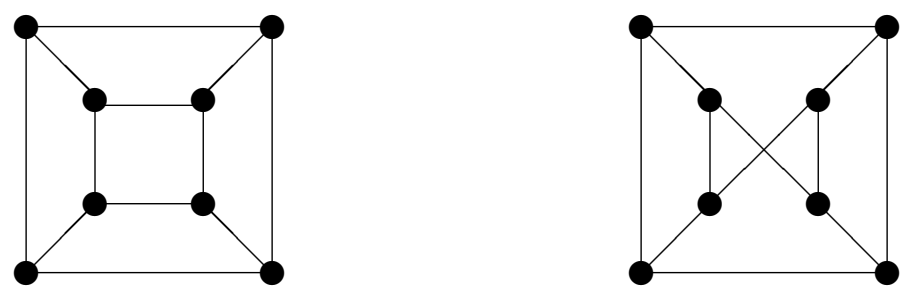

Figure 2.6

Now we are ready to complete the proof of Theorem 2.1.1. By the claim and by Lemmas 2.2 .1 and 2.2.2, we assume that $n \geq 12$ and that $G$ is triangle free. We will distinguish the following two subcases.

Subcase 3.1 $G$ has a nontrivial 3-cut.

Let $T=\left\{e_{1}, e_{2}, e_{3}\right\}$ be a nontrivial 3 edge cut of $G$. Since $G$ is a triangle free simple cubic graph, $G-T$ has only two components and each component of $G-T$ has at least 5 vertices. Let $H_{1}, H_{2}$ be the components of $G-T$ and assume that for $i=1,2,3, e_{i}=x_{i} y_{i}$ with $x_{1}, x_{2}, x_{3} \in V\left(H_{1}\right)$ and $y_{1}, y_{2}, y_{3} \in$ $V\left(H_{2}\right)$. 
Let $u$ and $v$ be two new vertices not in $V(G)$, let $H_{1}^{*}=H_{1} \cup\left\{x_{1} u, x_{2} u, x_{3} u\right\}$ and $H_{2}^{*}=H_{2} \cup\left\{y_{1} v, y_{2} v, y_{3} v\right\}$, and let $n_{i}=\left|V\left(H_{i}^{*}\right)\right|, i=1,2$. It follows that for $i=1,2, H_{i}$ is a 2 -connected simple cubic graph with $n_{i} \geq 6$ and $H_{i}^{*} \neq K_{4}$.

By induction, for $i=1,2, H_{i}^{*}$ has a cycle cover $\mathcal{C}_{i}$ with $\left|\mathcal{C}_{i}\right| \leq\left\lceil\frac{n_{i}}{4}\right\rceil$. Since $d_{H_{1}^{*}}(u)=3$ and since $d_{H_{2}^{*}}(v)=3$, we may assume that $\mathcal{C}_{1}$ has a cycle $W_{1}$ such that $x_{2} u, x_{3} u \in E\left(W_{1}\right)$ and $\mathcal{C}_{2}$ has a cycle $W_{2}$ such that $y_{2} v, y_{3} v \in E\left(W_{2}\right)$.

Let $C_{1}, C_{2}, \ldots, C_{s}$ be all the cycles in $\mathcal{C}_{1}$ such that $x_{1} u, x_{2} u \in \cap_{i=1}^{s} E\left(C_{i}\right)$, $D_{1}, D_{2}, \ldots, D_{t}$ be all the cycles in $\mathcal{C}_{1}$ such that $x_{1} u, x_{3} u \in \cap_{j=1}^{t} E\left(D_{j}\right)$, and $Z_{1}, Z_{2}, \ldots, Z_{r}$ be all the cycles in $\mathcal{C}_{1}$ such that $x_{2} u, x_{3} u \in \cap_{i=1}^{r} E\left(Z_{i}\right)$. (Note that $\left.W_{1} \in\left\{Z_{1}, Z_{2}, \ldots Z_{r}\right\}\right)$. Since $\mathcal{C}_{1}$ is a cycle cover of $G_{1}^{*}, s+t \geq 1$.

Since $\kappa\left(H_{2}^{*}\right) \geq 2, H_{2}^{*}$ has cycles $C, D$ such that $y_{1} v, y_{2} v \in E(C)$ and $y_{1} v, y_{3} v \in E(D)$. $\left(C\right.$ and $D$ may not be in $\left.\mathcal{C}_{2}\right)$. We modify all the cycles in $\mathcal{C}_{1}$ containing $u$ into cycles in $G$ by defining the following:

$$
\begin{aligned}
& C_{i}^{*}=\left(C_{i}-\right.\left.\left\{x_{1} u, x_{2} u\right\}\right) \cup\left(C-\left\{y_{1} v, y_{2} v\right\}\right) \cup\left\{y_{1} x_{1}, y_{2} x_{2}\right\}, i=1,2, \ldots, s, \\
& \quad(\text { if } s \geq 1), \\
& D_{j}^{*}=\left(D_{j}-\left\{x_{1} u, x_{3} u\right\}\right) \cup\left(D-\left\{y_{1} v, y_{3} v\right\}\right) \cup\left\{y_{1} x_{1}, y_{3} x_{3}\right\}, j=1,2, \ldots, t, \quad(\text { if } t \geq 1), \\
& Z_{k}^{*}=\left(Z_{k}-\left\{x_{2} u, x_{3} u\right\}\right) \cup\left(W_{2}-\left\{y_{2} v, y_{3} v\right\}\right) \cup\left\{y_{2} x_{2}, y_{3} x_{3}\right\}, k=1,2, \ldots, r .
\end{aligned}
$$

Similarly we can modify all cycles of $\mathcal{C}_{2}$ containing $v$ to the cycles of $G$. Thus we obtain a cycle cover $\mathcal{C}^{\prime}$ of $G$ consisting of all cycles of $\mathcal{C}_{1}$ and $\mathcal{C}_{2}$ which contain neither $u$ nor $v$, and all modified cycles of $\mathcal{C}_{1}$ and $\mathcal{C}_{2}$. It follows that $\left|\mathcal{C}^{\prime}\right| \leq\left|\mathcal{C}_{1}\right|+\left|\mathcal{C}_{2}\right|-1 \leq\left\lceil\frac{n_{1}}{4}\right\rceil+\left\lceil\frac{n_{2}}{4}\right\rceil-1 \leq\left\lceil\frac{n}{4}\right\rceil$, and so Theorem 1.1 follows in this subcase.

Subcase 3.2 G has no nontrivial 3-cut.

By Lemma 2.3 (ii), $G$ has two distinct edges $e_{1}=u_{1} v_{1}$ and $e_{2}=u_{2} v_{2}$ 
with

$N\left(u_{1}\right)=\left\{x_{1}, y_{1}, v_{1}\right\}, N\left(v_{1}\right)=\left\{x_{2}, y_{2}, u_{1}\right\}, N\left(u_{2}\right)=\left\{s_{1}, t_{1}, v_{2}\right\}, N\left(v_{2}\right)=\left\{s_{2}, t_{2}, u_{2}\right\}$.

such that $G^{*}=\left(\left(G-e_{1}\right)^{*}-e_{2}\right)^{*}$ is a 2-connected simple cubic graph.

Since $\left|V\left(G^{*}\right)\right|=n-4 \geq 8$, by induction, $G^{*}$ has a cycle cover $\mathcal{C}_{1}$ such that $\left|\mathcal{C}_{1}\right| \leq\left\lceil\frac{n-4}{4}\right\rceil$.

Assume that $C_{1}, C_{2}, \ldots, C_{s}, D_{1}, D_{2}, \ldots, D_{t}, Z_{1}, Z_{2}, \ldots, Z_{r}$ and $W_{1}, W_{2}, \ldots, W_{l}$ are all the cycles in $\mathcal{C}_{1}$ satisfying $x_{1} y_{1} \in \cap_{i=1}^{s} E\left(C_{i}\right), x_{2} y_{2} \in \cap_{j=1}^{t} E\left(D_{j}\right)$, $s_{1} t_{1} \in \cap_{k=1}^{r} E\left(Z_{k}\right)$ and $s_{2} t_{2} \in \cap_{\lambda=1}^{l} E\left(W_{\lambda}\right)$. Define

$$
\begin{aligned}
C_{i}^{*} & =\left(C_{i}-x_{1} y_{1}\right) \cup\left\{x_{1} u_{1}, u_{1} y_{1}\right\}, i=1,2, \ldots, s, \\
D_{j}^{*} & =\left(D_{j}-x_{2} y_{2}\right) \cup\left\{x_{2} v_{1}, v_{1} y_{2}\right\}, j=1,2, \ldots, t, \\
Z_{k}^{*} & =\left(Z_{k}-s_{1} t_{1}\right) \cup\left\{s_{1} u_{2}, u_{2} t_{1}\right\}, k=1,2, \ldots, r, \\
W_{\lambda}^{*} & =\left(W_{\lambda}-s_{2} t_{2}\right) \cup\left\{s_{2} v_{2}, v_{2} t_{2}\right\}, \lambda=1,2, \ldots, l .
\end{aligned}
$$

As $\kappa(G) \geq 2, G$ has a cycle $C$ with $u_{1} v_{1}, u_{2} v_{2} \in E(C)$.

Let

$$
\begin{aligned}
\mathcal{C} & =\mathcal{C}_{1}-\left\{C_{1}, C_{2}, \ldots, C_{s}, D_{1}, D_{2}, \ldots, D_{t}, Z_{1}, Z_{2}, \ldots, Z_{r}, W_{1}, W_{2}, \ldots, W_{l}\right\} \\
& \cup\left\{C_{1}^{*}, C_{2}^{*}, \ldots, C_{s}^{*}, D_{1}^{*}, D_{2}^{*}, \ldots, D_{t}^{*}, Z_{1}^{*}, Z_{2}^{*}, \ldots, Z_{r}^{*}, W_{1}^{*}, W_{2}^{*}, \ldots, W_{l}^{*}, C\right\} .
\end{aligned}
$$

Then $\mathcal{C}$ is a cycle cover of $G$ with $|\mathcal{C}|=\left|\mathcal{C}_{1}\right|+1 \leq\left\lceil\frac{n-4}{4}\right\rceil+1 \leq\left\lceil\frac{n}{4}\right\rceil$.

This completes the proof of Theorem 2.1.1. 


\section{Chapter 3}

\section{Hamiltonian Connected Line Graphs}

\subsection{Introduction}

The edge-connectivity of a graph $G$ is denoted by $\kappa^{\prime}(G)$. For a vertex $v \in$ $V(G), d_{G}(v)$ denotes the degree of $v$ in $G$. We use $H \subseteq G(H \subset G)$ to denote the fact that $H$ is a subgraph of $G$ (proper subgraph of $G$ ). If $X \subseteq E(G)$ is an edge subset, then $G[X]$ denotes the subgraph of $G$ induced by the edge subset $X$. If $H \subset G$, then for an edge subset $X \subseteq E(G)-E(H)$, we write $H+X$ for $G[E(H) \cup X]$. When $X=\{e\}$, we also use $H+e$ for $H+\{e\}$.

Let $X \subseteq E(G)$. The contraction $G / X$ is obtained from $G$ by contracting each edge of $X$ and deleting the resulting loops. If $H \subseteq G$, we write $G / H$ for $G / E(H)$. Note that even if $G$ is a simple graph, contracting some edges of $G$ may result in a graph with multiple edges. Note that any subset $X \subseteq$ $E(G / H)$ can also be viewed as a subset in $E(G)$. A connected graph with 
at least two vertices is called a nontrivial graph.

Let $O(G)$ denote the set of odd degree vertices of $G$. A graph $G$ is eulerian if $O(G)=\emptyset$ and $G$ is connected. A graph $G$ is supereulerian if $G$ has a spanning eulerian subgraph. In particular, $K_{1}$ is both eulerian and supereulerian. Pulleyblank indicated that determining if a graph $G$ is supereulerian, even within the family of planar graphs, is NP-complete (see [44]). For the literature of supereulerian subgraph, see the survey of Catlin [11] and its update [16].

A subgraph $H$ of a graph $G$ is dominating if $G-V(H)$ is edgeless. A dominating eulerian subgraph is also called a DES. For an integer $i \geq 1$, define

$$
D_{i}(G)=\{v \in V(G): d(v)=i\}
$$

There is a close relationship between dominating eulerian subgraphs in graphs and Hamilton cycles in $L(G)$.

Theorem 3.1.1 (Harary and Nash-Williams [29]) Let $G$ be a graph with $|E(G)| \geq 3$. Then $L(G)$ is hamiltonian if and only if $G$ has a DES.

A graph $G$ is hamiltonian connected if for every pair of vertices $u, v \in$ $V(G), G$ has a spanning $(u, v)$-path. We view a trail of $G$ as a vertex-edge alternating sequence

$$
v_{0}, e_{1}, v_{1}, e_{2}, \cdots, e_{k}, v_{k}
$$

such that all the $e_{i}$ 's are distinct and such that for each $i=1,2, \cdots, k, e_{i}$ is incident with both $v_{i-1}$ and $v_{i}$. All the vertices in $v_{1}, v_{2}, \cdots, v_{k-1}$ are internal vertices of trail in (3.1). 
For edges $e^{\prime}, e^{\prime \prime} \in E(G)$, an $\left(e^{\prime}, e^{\prime \prime}\right)$-trail of $G$ is a trail $T$ of $G$ whose first edge is $e^{\prime}$ and whose last edge is $e^{\prime \prime}$. (Thus the trail $T$ in (3.1) is an $\left(e_{1}, e_{k}\right)$-trail). A dominating $\left(e^{\prime}, e^{\prime \prime}\right)$-trail of $G$ is an $\left(e^{\prime}, e^{\prime \prime}\right)$-trail $T$ of $G$ such that every edge of $G$ is incident with an internal vertex of $T$; and a spanning $\left(e^{\prime}, e^{\prime \prime}\right)$-trail of $G$ is a dominating $\left(e^{\prime}, e^{\prime \prime}\right)$-trail $T$ of $G$ such that $V(T)=V(G)$. Arguing by a similar argument in the proof of Theorem 3.1.1, one can obtain the theorem for hamiltonian connected line graphs.

Theorem 3.1.2 Let $G$ be a graph with $|E(G)| \geq 3$. Then $L(G)$ is hamiltonian connected if and only if for any pair of edges $e^{\prime}, e^{\prime \prime} \in E(G), G$ has a dominating $\left(e^{\prime}, e^{\prime \prime}\right)$-trail.

Thomassen [47] conjectured that every 4-connected line graph is hamiltonian. Using Theorem 3.1.2, Zhan proved two results related to this conjecture.

Theorem 3.1.3 (Zhan, [56] and [57]) Let $G$ be a graph. Each of the following holds.

(i) ([56]) If $\kappa^{\prime}(G) \geq 4$, then $L(G)$ is hamiltonian connected.

(ii) ([57]) If $L(G)$ is 7-connected, then $L(G)$ is hamiltonian connected.

Utilizing a spanning tree packing theorem by Nash-Williams [43] and Tutte [50], Catlin, among others, was able to prove a relationship between the spanning tree packing number and the edge connectivity of a graph.

Theorem 3.1.4 (Catlin[14]) Let $G$ be a graph and let $k \geq 1$ be an integer. The following are equivalent.

(i) $\kappa^{\prime}(G) \geq 2 k$. 
(ii) For any edge subset $X \subset E(G)$ with $|X| \leq k, G-X$ has at least $k$ edge-disjoing spanning trees.

In particular, every 4-edge-connected graph has 2 edge-disjoint spanning trees. Seeing this, Catlin and Lai improved Theorem 3.1.3(i).

Theorem 3.1.5 (Catlin et al [5]) Let $G$ be a graph with 2 edge-disjoint spanning trees. Then $L(G)$ is hamiltonian connected if and only if $L(G)$ is 3-connected.

Let $G$ be a nontrivial graph (that is, $E(G) \neq \emptyset$,) that is not a path. Define $L^{0}(G)=G$, and for integer $k>0$, define the repeated line graph $L^{k}(G)=L\left(L^{k-1}(G)\right)$.

Theorem 3.1.6 (Chen et al, [19]) If $L^{2}(G)$ is 4-connected, then $L^{2}(G)$ is hamiltonian.

To further improve Theorem 3.1.3(i) and Theorem 3.1.6, we continue the investigation on 3-edge-connected graph which would have a hamiltonian connected line graph, and we also ask if every 4-connected $L^{2}(G)$ is hamiltonian connected. The purpose of this paper is to seek answers to these questions.

We say that an edge $e \in E(G)$ is subdivided when it is replaced by a path of length 2 whose internal vertex, denoted $v(e)$, has degree 2 in the resulting graph. The process of taking an edge $e$ and replacing it by that length 2 path is called subdividing e. For a graph $G$ and edges $e^{\prime}, e^{\prime \prime} \in E(G)$, let $G\left(e^{\prime}\right)$ denote the graph obtained from $G$ by subdividing $e^{\prime}$, and let $G\left(e^{\prime}, e^{\prime \prime}\right)$ denote 
the graph obtained from $G$ by subdividing both $e^{\prime}$ and $e^{\prime \prime}$. Thus,

$$
V\left(G\left(e^{\prime}, e^{\prime \prime}\right)\right)-V(G)=\left\{v\left(e^{\prime}\right), v\left(e^{\prime \prime}\right)\right\}
$$

From the definitions, one immediately has the following observation.

Lemma 3.1.7 For a graph $G$ and edges $e^{\prime}, e^{\prime \prime} \in E(G)$, if $G\left(e^{\prime}, e^{\prime \prime}\right)$ has a spanning $\left(v\left(e^{\prime}\right), v\left(e^{\prime \prime}\right)\right)$-trail, then $G$ has a spanning $\left(e^{\prime}, e^{\prime \prime}\right)$-trial.

Note that that $G$ has a spanning $\left(e^{\prime}, e^{\prime \prime}\right)$-trail does not imply that $G$ has a spanning $\left(v\left(e^{\prime}\right), v\left(e^{\prime \prime}\right)\right)$-trail. Let $\mathcal{F}$ denote the set of connected graphs such that a graph $G \in \mathcal{F}$ if and only if each of the following holds:

(F1) If $X$ is an edge cut of $G$ with $|X| \leq 3$, then there exists a vertex $v \in D_{|X|}(G)$ such that $X$ consists of all the edges incident with $v$ in $G$, and

(F2) for every $v \in D_{3}(G), v$ lies in a $k$-cycle $C_{v}$ of $G$, where $2 \leq k \leq 3$,

Theorem 3.1.8 Let $G \in \mathcal{F}$. If $\kappa^{\prime}(G) \geq 3$, then for every pair of edges $e^{\prime}, e^{\prime \prime} \in E(G)$ we have

(i) $G\left(e^{\prime}, e^{\prime \prime}\right)$ is collapsible and

(ii) G has a spanning $\left(v\left(e^{\prime}\right), v\left(e^{\prime \prime}\right)\right)$-trail.

This theorem has a number of corollaries.

Corollary 3.1.9 Let $G$ be a graph such that the set of neighbors of each vertex of degree 3 in $G$ is not an independent set. If $L(G)$ is 4-connected, $L(G)$ is hamiltonian connected.

Corollary 3.1.10 (Kriesell, [37]) If $G$ is a $K_{1,3}$-free graph and if $L(G)$ is 4-connected, then $L(G)$ is hamiltonian connected. 
Corollary 3.1.11 (Zhan, [57]) If $\kappa^{\prime}(G) \geq 4$, then $L(G)$ is hamiltonian connected.

Corollary 3.1.12 If $L^{2}(G)$ is 4-connected, then $L^{2}(G)$ is hamiltonian connected.

Let $C_{4}$ denote a 4-cycle in $K_{5}$. The graph $K_{5}-E\left(C_{4}\right)$ is called an hourglass. A graph $G$ is hourglass free if $G$ does not have a induced subgraph isomorphic to $K_{5}-E\left(C_{4}\right)$.

Corollary 3.1.13 (Broersma, Kriesell and Ryjacek, [9]) Every 4-connected hourglass free line graph is hamiltonian connected.

In Section 2, we discuss Catlin's reduction method which will be needed in the proof of the main result. In Section 3, we prove Theorem 3.1.8. The last section is devoted to the generalizations of Theorem 3.1.8 and to applications of the main results.

\subsection{Catlin's Reduction Method}

In [12] Catlin defined collapsible graphs. A subgraph $\Gamma$ of $G$ is called an $R$-subgraph if both $O(\Gamma)=R$ and $G-E(\Gamma)$ is connected. A graph $G$ is collapsible if for any even subset $R$ of $V(G), G$ has an $R$-subgraph. Catlin showed in [12] that every vertex of $G$ lies in a unique maximal collapsible subgraph of $G$. The reduction of $G$ is obtained from $G$ by contracting all maximal collapsible subgraph. A graph $G$ is reduced if $G$ has no nontrivial collapsible subgraphs. A nontrivial vertex in the reduction of $G$ is a vertex 
which is the contraction image of a nontrivial connected subgraph of $G$. Note that if $G$ has an $O(G)$-subgraph $\Gamma$, then $G-E(\Gamma)$ is a spanning eulerian subgraph of $G$. Therefore, every collapsible graph is supereulerian. We summerize some results on Catlin's reduction method and other related facts as follows.

Theorem 3.2.1 Let $G$ be a graph and let $H$ be a collapsible subgraph of $G$. Let $v_{H}$ denote the vertex onto which $H$ is contracted in $G / H$. Each of the following holds.

(i) (Catlin, Theorem 3 of [12]) $G$ is collapsible (supereulerian, respectively) if and only if $G / H$ is collapsible (supereulerian, respectively). In particular, $G$ is supereulerian if and only if the reduction of $G$ is supereulerian; and $G$ is collapsible if and only if the reduction of $G$ is $K_{1}$.

(ii) If $G$ is collapsible, then for any pair of vertices $u, v \in V(G), G$ has a spanning $(u, v)$-trail.

(iii) For vertices $u, v \in V(G / H)-\left\{v_{H}\right\}$, if $G / H$ has a spanning $(u, v)$ trail, then $G$ has a spanning $(u, v)$-trail.

(iv) 2-cycles and 3-cycles are collapsible.

Proof. (ii). Let $R=(O(G) \cup\{u, v\})-(O(G) \cap\{u, v\})$. Then $|R|$ is even. Let $\Gamma_{R}$ be an $R$-subgraph of $G$. Note that $G-E\left(\Gamma_{R}\right)$ is connected and that $u$ and $v$ are the only two vertices of odd degrees in $G-E\left(\Gamma_{R}\right)$. Thus $G-E\left(\Gamma_{R}\right)$ is a spanning $(u, v)$-trail of $G$.

(iii). This follows from Theorem 2(i).

(iv). This follows from definition immediately. 
Jaeger in [35] showed that if $G$ has two edge-disjoint spanning trees, then $G$ is supereulerian. This result is later improved by Catlin (Theorem 7 in [12]). Defining $F(G)$ to be the minimum number of additional edges that must be added to $G$ so that the resulting graph has two edge-disjoint spanning tree, Catlin [12] and Catlin et al [15] improve Jaeger's result. We put these former results in the following theorem.

Theorem 3.2.2 Let $G$ be a graph. Each of the following holds.

(i) (Jaeger, [35]) If $F(G)=0$, then $G$ is supereulerian.

(ii) (Catlin, Theorem 7 in [12]) If $F(G) \leq 1$ and if $G$ is connected, then $G$ is collapsible if and only if $G$ cannot be contractible to a $K_{2}$.

(iii) (Catlin, Han and Lai, Theorem 1.5 in [15]) If $F(G) \leq 2$ and if $G$ is connected, then $G$ is collapsible if and only if the reduction of $G$ is not a $K_{2}$ nor a $K_{2, s}$ for some integer $s \geq 1$.

In order to apply Theorem 3.2.2 in our proofs, we also need the following observations.

Lemma 3.2.3 Let $G$ be a graph. Each of the following holds.

(i) For any $e \in E(G), F(G(e)) \leq F(G)+1$.

(ii) $F(G) \leq F(G / e)+1$.

Proof. (i). Suppose that $X$ is a set of edges not in $G$ such that $G+X$ has two disjoint spanning trees $T_{1}$ and $T_{2}$. Assume that $e=v_{1} v_{2}$. Then at most one of them, say $T_{1}$, contains $e$ and hence $T_{2}$ does not contain $e$. Therefore, one needs at most one more edge $\left(v_{1} v(e)\right.$, for example) to $X$ so that $G+\left(X \cup\left\{v_{1} v(e)\right\}\right)$ has 2 edge-disjoint spanning trees. 
(ii). Let $X$ be a set of additional edges such that $G / e+X$ has 2 edgedisjoint spanning trees. Let $e^{\prime}$ be an edge not in $G$ but parallel to $e$. Then $(G+X)+e^{\prime}$ will have 2 edge-disjoint spanning trees.

\subsection{Proof of Thoerem 3.1.8}

We shall prove some lemmas. Let $G \in \mathcal{F}$ be a 3-edge-connected graph. For each $v \in D_{3}(G)$, fix a cycle $C_{v}$ such that $v \in V\left(C_{v}\right)$ and such that $2 \leq\left|V\left(C_{v}\right)\right| \leq 3$. Let

$$
W(G)=\bigcup_{v \in D_{3}(G)} C_{v}
$$

We have the following observations.

Lemma 3.3.1 Let $G \in \mathcal{F}$ be a 3-edge-connected graph. Then $G / W(G)$ is 4-edge-connected.

Proof. Let $X \subset E(G / W(G))$ be an edge cut. Note that $X$ is also an edge-cut of $G$ and $X \subseteq E(G)-W$. If $|X| \leq 3$, then since $\kappa^{\prime}(G) \geq 3$, one has $|X|=3$. By (F1), there exists a verex $v \in D_{3}(G)$ such that $X$ consists of the three edges incident with $v$ in $G$. By $(\mathrm{F} 2), G$ has a cycle $C_{v}$ containing two edges in $X$ such that $E\left(C_{v}\right) \subseteq W(G)$, contrary to the fact that $X \cap W(G)=\emptyset$. Hence one must have $|X| \geq 4$.

Lemma 3.3.2 If $G \in \mathcal{F}$ and if $e \in E(G)$, then $G / e \in \mathcal{F}$.

Proof. By the definition of contraction, $G / e$ is connected. If $X \subset E(G / e)$ is an edge cut, then $X$ is also an edge cut of $G$, and so $G / e$ satisfies (F1). 
Suppose that $v_{e}$ is the contraction image of $e$. If $v_{e} \in D_{3}(G / e)$, then since $G \in \mathcal{F}$, the three edges incident with $v_{e}$ in $G / e$ are incident with a vertex $v \in D_{3}(G)$, which leaves no room for $e$. Hence $G / e$ also satisfies (F2).

Lemma 3.3.3 Let $G$ be a graph. If $\kappa^{\prime}(G) \geq 4$, then for any $e^{\prime}, e^{\prime \prime} \in E(G)$, (i) $G\left(e^{\prime}, e^{\prime \prime}\right)$ has 2 edge-disjoint spanning trees, and (ii) $G\left(e^{\prime}, e^{\prime \prime}\right)$ has a spanning $\left(v\left(e^{\prime}\right), v\left(e^{\prime \prime}\right)\right)$-trail.

Proof. By Theorem 3.1.5, $G-\left\{e^{\prime}, e^{\prime \prime}\right\}$ has two edge-disjoint spanning trees, and so $G\left(e^{\prime}, e^{\prime \prime}\right)$ also has 2 edge-disjoint spanning trees. This proves Lemma 3.3.3(i).

If $G\left(e^{\prime}, e^{\prime \prime}\right)$ has 2 edge-disjoint spanning trees, then by Theorem 3.2.2(ii) or (iii), $G\left(e^{\prime}, e^{\prime \prime}\right)$ is collapsible, and so Lemma 3.3.3(ii) follows from Theorem 2(ii).

Lemma 3.3.4 Let $G \in \mathcal{F}$ such that $\kappa^{\prime}(G) \geq 3$ and such that $\left|D_{3}(G)\right| \leq 2$. Then $F(G)=0$.

Proof. Let $v \in D_{3}(G)$ and let $v_{1}, v_{2}, v_{3}$ be the three vertices in $G$ that are adjacent to $v$, and let $C_{v}=v v_{1} v_{2} v$ be a 3-cycle in $G$ containing $v$. Let $G^{\prime}=G /\left(W(G)-E\left(C_{v}\right)\right)$.

By Lemma 3.3.1, $G / W(G)$ is 4-edge-connected. By Theorem 3.1.5, $G / W(G)-$ $e_{3}$ has 2 edge-disjoint spanning trees $T_{1}$ and $T_{2}$, where $e_{3}=v v_{3}$. Both $T_{1}$ and $T_{2}$ can be viewed as edge induced forests in $G^{\prime}$. Note that for each $i=1,2, T_{i}$ contains at least $\left|V\left(G^{\prime}\right)\right|-2$ vertices in $V\left(G^{\prime}-v\right)$, and has two components, $T_{i}^{\prime}$ and $T_{i}^{\prime \prime}$, with $v_{1} \in V\left(T_{i}^{\prime}\right)$ and $v_{2} \in V\left(T_{i}^{\prime \prime}\right)$. (It is possible that $T_{i}^{\prime}$ or $T_{i}^{\prime \prime}$ is a trivial tree). 
By symmetry, we may assume either $v_{3} \in V\left(T_{1}^{\prime \prime}\right) \cap V\left(T_{2}^{\prime \prime}\right)$, or $v_{3} \in V\left(T_{1}^{\prime \prime}\right) \cap$ $V\left(T_{2}^{\prime}\right)$. In either case, $T_{1}+\left\{v v_{3}, v v_{1}\right\}$ and $T_{2}+\left\{v_{1} v_{2}, v v_{2}\right\}$ are 2 edge-disjoint spanning trees of $G$.

If $\left|D_{3}(G)\right|=2$, one can do the same for the other vertex in $D_{3}(G)$. Theorem 3.1 .5 allows us to remove at least 2 edges $e_{3}, e_{3}^{\prime}$ from $G / W(G)$ while keeping $F\left(G / W(G)-\left\{e_{3}, e_{3}^{\prime}\right)\right)=0$.

\section{Proof of Theorem 3.1.8.}

(i) Let $e^{\prime}, e^{\prime \prime}$ be a pair of edges in $G$. We argue by induction on $|V(G)|$ to prove Theorem 3.1.8, which is trivial when $|V(G)| \leq 4$. If $G$ has a collapsible subgraph $H$ such that each of $e^{\prime}$ and $e^{\prime \prime}$ has at most one end in $V(H)$, then one can argue by Theorem 2(i) and apply induction on $G\left(e^{\prime}, e^{\prime \prime}\right) / H$ to obtain that $G\left(e^{\prime}, e^{\prime \prime}\right)$ is collapsible. Hence we assume that for any collapsible subgraph $H$ of $G$,

at least one of $e^{\prime}$ and $e^{\prime \prime}$ has both ends in $V(H)$

If $e^{\prime}, e^{\prime \prime} \notin W(G)$, then by Lemma 3.3.3(i) and by Lemma 3.3.1, $G\left(e^{\prime}, e^{\prime \prime}\right) / W(G)$ has 2-edge-disjoint spanning trees. By Theorem 3.2.2(ii), $G\left(e^{\prime}, e^{\prime \prime}\right) / W(G)$ is collapsible. By Theorem 2(i) and (iv) $G\left(e^{\prime}, e^{\prime \prime}\right)$ is collapsible. Hence we assume that $\left\{e^{\prime}, e^{\prime \prime}\right\} \cap W(G) \neq \emptyset$. By (3.3) and by Theorem 2(iv), and by the definition of $\mathcal{F}$, we must have $\left|D_{3}(G)\right| \leq 2$.

By Lemma 3.3.4, $F(G)=0$, and so by Lemma 3.2.3(i), $F\left(G\left(e^{\prime}, e^{\prime \prime}\right)\right) \leq 2$. It follows by Theorem 3.2.2(iii) that the reduction of $G\left(e^{\prime}, e^{\prime \prime}\right)$ is either a $K_{1}$, or a $K_{2}$, or a $K_{2, t}$ for some $t \geq 1$.

If the reduction of $G\left(e^{\prime}, e^{\prime \prime}\right)$ is $K_{1}$, then $G\left(e^{\prime}, e^{\prime \prime}\right)$ is collapsible. Thus we assume that the reduction of $G\left(e^{\prime}, e^{\prime \prime}\right)$ is not $K_{1}$ to derive a contradiction. 
By Lemma 3.3.1, $G / W(G)$ is 4-edge-connected. Thus $G\left(e^{\prime}, e^{\prime \prime}\right)$ cannot have a cut edge and the reduction of $G\left(e^{\prime}, e^{\prime \prime}\right)$ must be a $K_{2, t}$ for some $t \leq 2$. It follows that the reduction of $G\left(e^{\prime}, e^{\prime \prime}\right)$ must be a $K_{2,2}$, and so we denote the reduction of $G\left(e^{\prime}, e^{\prime \prime}\right)$ by $C_{4}$. Since $G / W(G)$ is 4-edge-connected, two nonadjacent vertices of this $C_{4}$ must be $\left\{v\left(e^{\prime}\right), v\left(e^{\prime \prime}\right)\right\}$. It follows that $\left\{e^{\prime}, e^{\prime \prime}\right\}$ is an edge cut of $G$, contrary to the assumption that $\kappa^{\prime}(G) \geq 3$.

(ii) It follows from (i) and Theorem 2(ii).

\subsection{Generalizations and Applications}

For the purpose of applications to hamiltonian line graph, the requirement that $\kappa^{\prime}(G) \geq 3$ in Theorem 3.1.8 can be relaxed, in view of Lemma 3.1.2.

Let $G$ be a graph. For each $v \in D_{2}(G)$, fix exactly one edge $e_{v}$ that is incident with $v$ in $G$, and let $W^{\prime}(G)=\cup\left\{e_{v}: v \in D_{2}(G)\right\}$. Define $\tilde{G}=G / W^{\prime}(G)$. Also, define $W^{\prime \prime}(G)=E(G)-E\left(G-D_{1}(G)\right)$ denote the set of edges that are incident with a vertex in $D_{1}(G)$.

Lemma 3.4.1 Let $G$ be a graph such that $G-D_{1}(G)$ is 2-edge-connected and such that $D_{2}(G)$ is an independent set. Then any spanning trail of $\tilde{G}-D_{1}(\tilde{G})$ is a dominating trail of $G$.

Proof. Let $L$ denote a spanning trail of $\tilde{G}-D_{1}(\tilde{G})$. Note that $D_{1}(\tilde{G})=$ $D_{1}(G)$. Therefore, any vertex $v \in V(G)-V(L)$ must be a vertex in $D_{1}(G) \cup$ $D_{2}(G)$. If $v \in D_{1}(G)$, then since $G-D_{1}(G)$ is 2-edge-connected, $v$ must be incident to a vertex in $V\left(\tilde{G}-D_{1}(\tilde{G})\right)=V(L)$; if $v \in D_{2}(G)$, then since $D_{2}(G)$ is an independent set in $G$ and since $G-D_{1}(G)$ is 2-edge-connected, 
$v$ must be incident with a vertex in $V(L)$ as well. It follows that $G-V(L)$ is edgeless and so $L$ is a dominating trail of $G$.

Theorem 3.4.2 Let $G \in \mathcal{F}$ be a graph such that $\kappa^{\prime}\left(\tilde{G}-D_{1}(\tilde{G})\right) \geq 3$. Then for any $e^{\prime}, e^{\prime \prime} \in E(G), G$ has a dominating $\left(e^{\prime}, e^{\prime \prime}\right)$-trail.

Proof. If $e^{\prime}, e^{\prime \prime}$ are two edges incident with a vertex $v$ of degree 2 of $G$, let $e^{\prime}=$ $x v, e^{\prime \prime}=v y$. We assume that $x v \in W^{\prime}(G)$. Then $e=v y \in E\left(\tilde{G}-D_{1}(\tilde{G})\right)$. We can think that $e^{\prime}, e^{\prime \prime}$ are obtained by subdividing $e$. By Theorem 1.8 (i) $\left(\tilde{G}-D_{1}(\tilde{G})\right)\left(e^{\prime}, e^{\prime \prime}\right)$ is collapsible. Thus $\left(\tilde{G}-D_{1}(\tilde{G})\right)\left(e^{\prime}, e^{\prime \prime}\right)$ is supereulerian and hence $G$ has a dominating $\left(e^{\prime}, e^{\prime \prime}\right)$-trial. So suppose $e^{\prime}, e^{\prime \prime}$ are not incident with the same vertex of degee 2 in $G$. By the definition of $W^{\prime}(G)$, we can choose $W^{\prime}(G)$ such that $\left\{e^{\prime}, e^{\prime \prime}\right\} \cap W^{\prime}(G)=\emptyset$. Thus we assume that $e^{\prime}, e^{\prime \prime} \in E\left(\tilde{G}-D_{1}(\tilde{G})\right.$. Then by Theorem 3.1.8, $\left(\tilde{G}-D_{1}(\tilde{G})\right)\left(e^{\prime}, e^{\prime \prime}\right)$ has a spanning $v\left(e^{\prime}\right), v\left(e^{\prime \prime}\right)$-trail, and so by Lemma 3.4.1 and by Lemma 3.1.7, $G$ has a dominating $\left(e^{\prime}, e^{\prime \prime}\right)$-trail. Thus we may assume that $e^{\prime} \in W^{\prime \prime}(G)$. Let $v^{\prime}$ denote the vertex in $D_{1}(G)$ incident with $e^{\prime}$. Note that either $e^{\prime \prime} \in W^{\prime \prime}(G)$ or $e^{\prime \prime} \in E\left(G-D_{1}(G)\right)$.

Suppose first that $e^{\prime \prime} \in W^{\prime \prime}(G)$ and let $v^{\prime \prime}$ be the vertex in $D_{1}(G)$ incident with $e^{\prime \prime}$. By Lemma 3.3.1, $\left(\tilde{G}-D_{1}(\tilde{G})\right) / W(G)$ is 4-edge-connected; and so by Theorem 3.2.2, $\left(\tilde{G}-D_{1}(\tilde{G})\right) / W(G)$ is collapsible. By Theorem 2(i) and (v), $\tilde{G}-D_{1}(\tilde{G})$ is also collapsible, and so by Theorem $2(\mathrm{ii}), \tilde{G}-D_{1}(\tilde{G})$ has a spanning $\left(v^{\prime}, v^{\prime \prime}\right)$-trail. It follows by Lemma 3.4.1 that $G$ has a dominating $\left(e^{\prime}, e^{\prime \prime}\right)$-trail.

Hence $e^{\prime \prime} \in E\left(G-D_{1}(G)\right)$. Let $u=v\left(e^{\prime \prime}\right)$. By Lemma 3.3.1, $(\tilde{G}-$ $\left.D_{1}(\tilde{G})\right) / W(G)$ is 4-edge-connected; and so by Lemma 3.2 .3 and by Theorem 3.2.2(ii), $\left(\tilde{G}-D_{1}(\tilde{G})\right)\left(e^{\prime \prime}\right) / W(G)$ is collapsible. By Theorem 2(i) and (v), 
$\left(\tilde{G}-D_{1}(\tilde{G})\right)\left(e^{\prime \prime}\right)$ is also collapsible, and so by Theorem $2(\mathrm{ii}),\left(\tilde{G}-D_{1}(\tilde{G})\right)\left(e^{\prime \prime}\right)$ has a spanning $\left(v^{\prime}, v^{\prime \prime}\right)$-trail. It follows by Lemma 3.4.1 that $G$ has a dominating $\left(e^{\prime}, e^{\prime \prime}\right)$-trail.

Lemma 3.4.3 Let $G$ be a graph such that $L(G)$ is 4-connected, then each of the following holds.

(i) G satisfies (F1).

(ii) $\kappa^{\prime}\left(\tilde{G}-D_{1}(\tilde{G})\right) \geq 3$.

Proof. Let $X \subset E(G)$ be an edge cut of $G$. If $G-X$ has two nontrivial components, then $X$ corresponds to a vertex cut of $L(G)$, and so $|X| \geq 4$, by the assumption that $\kappa(L(G)) \geq 4$. Hence $G$ satisfies $(\mathrm{F} 1)$.

Since every edge cut in $\tilde{G}-D_{1}(\tilde{G})$ is an edge cut in $G$ that either separate a vertex in $D_{3}(G)$ with other vertices in $G$, or corresponds to a vertex cut in $L(G)$, it follows again by $\kappa(L(G)) \geq 4$ that $\kappa^{\prime}\left(\tilde{G}-D_{1}(\tilde{G})\right) \geq 3$.

Proof of Corollary 3.1.9. Since the set of the neighbors of each vertex of degree 3 is not independent set, $G$ satisfies (F2). By Lemma 3.4.3, $G \in \mathcal{F}$ and $\kappa^{\prime}\left(\tilde{G}-D_{1}(\tilde{G})\right) \geq 3$. By Theorem 3.4.2 and Lemma 3.1.2, $L(G)$ is hamiltonian connected.

Proof of Corollary 3.1.10. Since $G$ is $K_{1,3}$-free, the set of the neighbors of each vertex of degree 3 is not independent set. Thus Corollary 3.1.10 follows from Corollary 3.1.9.

Proof of Corollary 3.1.11. Since $\kappa^{\prime}(G) \geq 4, d_{G}(v) \geq 4$ for every $v \in V(G)$. Thus Corollaty 3.1.11 follows from Corollary 3.1.9. 
Proof of Corollary 3.1.12. It is well known that a line graph does not have a $K_{1,3}$ as an induced subgraph. Thus Corollary 3.1.12 follows from Corollary 3.1.10.

Proof of Corollary 3.1.13. We may assume that $L(G)$ is not a complete graph. By Lemma 3.4.3, $G$ satisfies $(\mathrm{F} 1)$ and $\kappa^{\prime}\left(\tilde{G}-D_{1}(\tilde{G})\right) \geq 3$. We shall show that $G$ aslo satisfies (F2).

By contradiction, there exists a $v \in D_{3}(G)$ with $v_{1}, v_{2}, v_{3} \in V(G)$ being three distinct vertices adjacent to $v$ in $G$, such that $v_{1}, v_{2}, v_{3}$ are mutually nonadjacent, and such that $v_{1} \notin D_{1}(G)$. By $\kappa(L(G)) \geq 4, d_{G}\left(v_{1}\right) \geq 3$. Let $v_{1} u, v_{1} u^{\prime}$ be two edges of $G$ such that $v \notin\left\{u, u^{\prime}\right\}$. It follows that the edges $\left\{v v_{1}, v v_{2}, v v_{3}, v_{1} u, v_{1} u^{\prime}\right\}$ induces an hourglass in $L(G)$, a contradiction.

Thus $G$ satisfies (F2), and so $G \in \mathcal{F}$. Therefore, Corollary 3.1.13 follows from Theorem 3.4.2 and Lemma 3.1.2. 


\section{Chapter 4}

\section{Circuits containing 12 vertices in 3-edge-connected graphs and Hamiltonian line graphs}

\subsection{Introduction}

As in $[8], \kappa(G), \kappa^{\prime}(G)$ and $d_{G}(v)$ denote the connectivity of $G$, the edgeconnectivity of $G$ and the degree of a vertex $v$ in $G$, respectively. Let $G$ be a graph. Let $O(G)$ denote the set of odd degree vertices of $G$. If $O(G)=\emptyset$ and if $G$ is connected, then $G$ is an Eulerian graph. Note that $K_{1}$ is Eulerian. An Eulerian subgraph of $G$ will be called a circuit. Hence a subgraph $H$ of $G$ is a circuit if and only if $H$ is a connected and every vertex of $H$ has even degree in $H$. A circuit $H$ is a dominating circuit of $G$ if $G-V(H)$ is edgeless.

Let $G$ be a graph and let $X \subseteq E(G)$. The contraction $G / X$ is the 
graph obtained from $G$ by identifying the two ends of each edge in $X$ and then deleting the resulting loops. A graph is trivial if it is edgeless. If $G_{0}=G / X$ and if every vertex of $G_{0}$ is a nontrivial vertex, then $G_{0}$ is a nontrivial contraction of $G$. If $H$ is a subgraph of $G$, then we write $G / H$ for $G / E(H)$. If $H$ is a connected subgraph of $G$, and if $v_{H}$ denotes the vertex in $G / H$ to which $H$ is contracted, then $H$ is called the preimage of $v_{H}$. A vertex $v$ in a contraction of $G$ is nontrivial if $v$ has a nontrivial preimage.

For a graph $G$, the line graph $L(G)$ has $E(G)$ as its vertex set, where two vertices in $L(G)$ are adjacent in $L(G)$ if and only if the corresponding edges are adjacent in $G$. The following relates dominating Eulerian subgraphs and Hamiltonian line graphs.

Theorem 4.1.1 (Harary and Nash-Williams [29]). Let $G$ be a graph with at least three edges. Then $L(G)$ is Hamiltonian if and only if $G$ has a dominating circuit.

In this chapter, we first study the existence of a circuit $H$ in a 3-edgeconnected graph $G$ such that $H$ contains a given set of vertices of $G$. We prove the following:

Theorem 4.1.2 Let $G$ be a 3-edge-connected graph and let $S \subseteq V(G)$ be a vertex subset such that $|S| \leq 12$. Then either $G$ has a circuit $H$ such that $S \subseteq V(H)$, or $G$ can be contracted to the Petersen graph in such a way that the preimage of each vertex of the Petersen graph contains at least one vertex in $S$.

If $G$ is a planar graph, we have 
Theorem 4.1.3 Let $G$ be a 3-edge-connected planar graph, and let $S \subseteq$ $V(G)$ be a vertex subset such that $|S| \leq 23$. Then there is a circuit in $G$ containing $S$.

When $G$ is a cubic graph, $\kappa(G)=\kappa^{\prime}(G)$ and every circuit $H$ of $G$ is a cycle of $G$. Therefore, Theorem 4.1.2 and Theorem 4.1.3 extend the following results in [5] and [1], respectively.

Theorem 4.1.4 (Bau and Holton [5]). Let $G$ be a 3-connected cubic graph and let $S \subseteq V(G)$ be a vertex subset such that $|S| \leq 12$. Then either $G$ has a cycle $H$ such that $S \subseteq V(H)$, or $G$ is contractible to the Petersen graph in such a way that the preimage of each vertex of the Petersen graph contains at least one vertex in $S$.

Theorem 4.1.5 (Aldred, Bau, Holton and McKay [1]). If $S$ is a set of at most 23 vertices in a 3-connected cubic planar graph $G$, then there is a cycle in $G$ containing $S$.

Let $G$ be the graph shown in Figure 1 below, where each $H$ is a single vertex ( See [32], page 243). If $S$ is the set of those 13 vertices marked by $H$, then there is no cycle through the 13 vertices. This shows that the requirement on 12 vertices in Theorem 4.1.2 and Theorem 4.1.4 can not be replaced by 13 vertices. The sharpness of Theorem 4.1.5 and Theorem 4.1.3 are demonstrated in [31] that there are 3-edge-connected (cubic) planar graphs in which there is a set of 24 vertices that do not lie on a common cycle. 


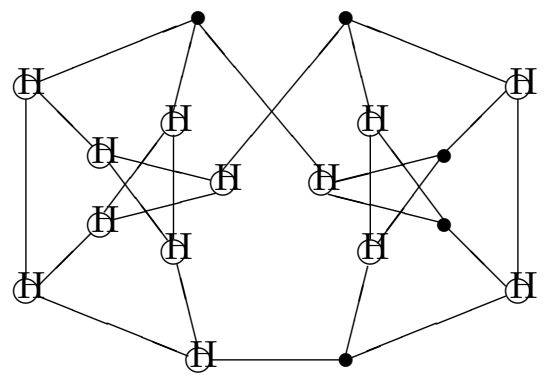

Figure 4.1

Next, we apply Theorem 4.1.2 to Hamiltonian line graphs. For an integer $i \geq 0$,

$$
D_{i}(G)=\left\{v \in V(G): d_{G}(v)=i\right\} .
$$

An edge $e \in E(G)$ is a pendant edge if $e$ is incident with a vertex in $D_{1}(G)$. The following was conjectured by Benhocine et al in 1986 [6], and proved by Veldman in 1994 [52].

Theorem 4.1.6 (Veldman [52]). Let $G$ be a simple graph on $n$ vertices such that $\kappa^{\prime}\left(G-D_{1}(G)\right) \geq 2$. If for every edge $u v \in E(G)$,

$$
d_{G}(u)+d_{G}(v)>\frac{2 n}{5}-2,
$$

then for $n$ large, $L(G)$ is Hamiltonian.

When the edge-connectivity is higher, the lower bound in (4.1) becomes lower as shown in [18]. The following was proved.

Theorem 4.1.7 (Chen and Lai [18]). Let $G$ be a simple graph on $n$ vertices such that $\kappa^{\prime}(G) \geq 3$. If for every edge $u v \in E(G)$,

$$
d_{G}(u)+d_{G}(v) \geq \frac{n}{6}-2,
$$


then for $n$ large, either $L(G)$ is Hamiltonian, or the Petersen graph is a nontrivial contraction of $G$.

Theorem 4.1.7 is an improvement of a previous result in [17] and [52]. The authors in [18] conjectured that the lower bound in Theorem 4.1.7 can be reduced to $n / 9-1$, with the conclusion that either $L(G)$ is Hamiltonian or $G$ is contractible to the Petersen graph. This conjecture, if proved, will be best possible, due to a construction using the Blanuša snarks [18].

Noting that (4.1) implies that

$$
\max \left\{d_{G}(u), d_{G}(v)\right\} \geq \frac{n}{5}-1, \text { for every edge } u v \in E(G)
$$

Lai considered (4.3) as a relaxation of (4.1), and made an improvement of Theorem 4.1.6.

Theorem 4.1.8 (Lai [40]). Let $G$ be a simple graph on $n$ vertices such that $\kappa^{\prime}\left(G-D_{1}(G)\right) \geq 2$. If (4.3) holds, then for $n$ large, either $L(G)$ is Hamiltonian, or (4.1) is violated and $G$ can be contracted to one of seven specified graphs.

Theorem 4.1.9 below extends Theorem 4.1.7.

Theorem 4.1.9 Let $G$ be a simple graph on $n$ vertices such that $\kappa^{\prime}(G-$ $\left.\left(D_{1}(G) \cup D_{2}(G)\right)\right) \geq 3$. If

$$
\max \left\{d_{G}(u), d_{G}(v)\right\} \geq \frac{n}{12}-\epsilon, \text { for every edge } u v \in E(G)
$$

where $\epsilon \geq 1$ is a constant, then for $n$ large, either $L(G)$ is Hamiltonian, or $G$ has the Petersen graph as a nontrivial contraction. 
Remark. $n / 12$ can not be replaced by $n / 13$ in Theorem 4.1.9. Let $r$ be an integer, and $n=13 r+5$. Let $G$ be the graph in Figure 1, where each $H$ is a complete graph $K_{r}$. Then $G$ is a graph of order $n=13 r+5$. Obviously, one can see that for every edge $u v \in E(G), \max \left\{d_{G}(u), d_{G}(v)\right\} \geq r-1=$ $\frac{n-5}{13}-1>\frac{n}{13}-2$. Although $G$ can be contracted to the Petersen graph, at least one vertex of the Petersen graph is a trivial contraction. Also $L(G)$ is not Hamiltonian. The statement of Theorem 4.1.9 is not true for this case. This shows that $\frac{n}{12}$ in (4.4) is the best possible.

In Section 2, we present Catlin's reduction method, which is the needed mechanism in the proofs. In Section 3, we assume the truth of Theorem 4.1.2 to prove Theorem 4.1.9. The last section will be devoted to the proofs of Theorem 4.1.2 and Theorem 4.1.3.

\subsection{Catlin's Reduction Method}

Let $G$ be a graph and let $F \subseteq V(G)$ be a vertex subset. A circuit $H$ of $G$ is called an $F$-circuit if $F \subseteq V(H)$. A graph $G$ is supereulerian if it has a $V(G)$-circuit. (See [11] for supereulerian graphs). Catlin [12] invented a reduction method to find a $V(G)$-circuit for given $G$.

A graph $G$ is collapsible if for every subset $R \subseteq V(G)$ with $|R|$ even, $G$ has a spanning connected subgraph $H_{R}$ such that $O\left(H_{R}\right)=R$. In [12], Catlin showed that every graph $G$ has a unique collection of maximal collapsible subgraphs $H_{1}, H_{2}, \cdots, H_{c}$. The reduction of $G$ is $G^{\prime}=G /\left(\cup_{i=1}^{c} H_{i}\right)$, the graph obtained from $G$ by contracting all nontrivial maximal collapsible subgraphs of $G$. A graph $G$ is reduced if the reduction of $G$ is $G$. Note that any subgraph 
of a reduced graph is reduced.

Theorem 4.2.1 (Catlin [12]). If $G$ is a reduced graph, then $G$ is simple and $K_{3}$-free, with $\delta(G) \leq 3$, and for any subgraph $H$ of $G$, either $H \in\left\{K_{1}, K_{2}\right\}$ or $|E(H)| \leq 2|V(H)|-4$.

Lemma 4.2.2 (Catlin [12]). Let $G$ be a graph. Let $L$ be a collapsible subgraph of $G$, and let $v_{L}$ be the vertex in $G / L$ to which $L$ is contracted, and $M \subseteq V(G)-V(L)$. Then $G$ has a circuit $H$ such that $M \cup V(L) \subseteq V(H)$ if and only if $G / L$ has a circuit $H^{\prime}$ such that $M \cup\left\{v_{L}\right\} \subseteq V\left(H^{\prime}\right)$.

Let $G$ be a graph. Let $v \in D_{2}(G)$ be a vertex which is incident with edges $e_{1}, e_{2}$. We say that the contraction $G / e_{1}$ is the resulting graph by eliminating a degree 2 vertex $v$. Define $\tilde{G}$ to be the graph obtained from $G-D_{1}(G)$ by eliminating all vertices in $D_{2}(G)$. Since deleting vertices in $D_{1}(G)$ is equivalent to contracting all the pendant edge in $G$, one can always view $\tilde{G}$ as a contraction of $G$.

Lemma 4.2.3 (Proposition 3.2 of [40]). Let $G$ be a graph and let $F \subseteq$ $V(G)-\left(D_{1}(G) \cup D_{2}(G)\right)$. The following are equivalent.

(i) G has an F-circuit.

(ii) $\tilde{G}$ has an F-circuit.

Let $H$ be a collapsible subgraph of $G$, and let $G^{\prime}=G / H$. Let $v_{H}$ denote the vertex in $G^{\prime}$ onto which the subgraph $H$ is contracted. Let $F \subseteq V(G)$ be a vertex subset. Define $F^{\prime} \subseteq V\left(G^{\prime}\right)$ such that

$$
F^{\prime}= \begin{cases}F & \text { if } F \cap V(H)=\emptyset, \\ (F-V(H)) \cup\left\{v_{H}\right\} & \text { if } F \cap V(H) \neq \emptyset .\end{cases}
$$


Lemma 4.2.4 Let $G$ be a graph and let $F \subseteq V(G)-\left(D_{1}(G) \cup D_{2}(G)\right)$. Let $H$ be a collapsible subgraph of $G$, and let $H^{\prime}$ denote the graph obtained from $H$ by eliminating all vertices in $D_{2}(G) \cap D_{2}(H)$. (Thus $H^{\prime}$ is a subgraph of $\tilde{G}$.) Let $G^{\prime \prime}$ denote $\tilde{G} / H^{\prime}$. The following are equivalent.

(i) $G$ has an $F$-circuit which is either disjoint from $H$, or contains every vertex of $H$.

(ii) $G^{\prime \prime}$ has an $F^{\prime}$-circuit.

Proof. Since $F \subseteq V(G)-\left(D_{1}(G) \cup D_{2}(G)\right)$, one can view $F \subseteq V(\tilde{G})$, and so $F^{\prime} \subseteq V\left(G^{\prime \prime}\right)$.

If $L$ is an $F$-circuit satisfying (i), and if $X$ is the edge subset such that $G^{\prime \prime}=G / X$, then $L /(X \cap E(L))$ is an $F^{\prime}$-circuit in $G^{\prime \prime}$.

Suppose that $G^{\prime \prime}$ has an $F^{\prime}$-circuit $L^{\prime}$. By Lemma 4.2 .3 and by Lemma 4.2.2, if $v_{H} \in V\left(L^{\prime}\right)$, then $G$ has an $F$-circuit $L$ that contains every vertex of $H$; if $v_{H} \notin V\left(L^{\prime}\right)$, then $G$ has an $F$-circuit that does not intersect $H$, and so (i) must hold.

\subsection{Proof of Theorem 4.1.9}

Throughout this section, we assume that Theorem 4.1.2 holds. Let $G$ be a simple graph with $n$ vertices. Following closely the method of [40], we consider the condition

$$
\max \left\{d_{G}(u), d_{G}(v)\right\} \geq \frac{n}{p}-\epsilon, \text { for every edge } u v \in E(G),
$$

where $\epsilon$ is a constant. Let $p \geq 2$ be an integer, and define

$$
J_{p}(G)=\left\{v \in V(G): d(v) \geq \frac{n}{p}-\epsilon\right\} .
$$


Lemma 4.3.1 Suppose that (4.5) holds for a simple graph $G$ with $n$ vertices. Let $\tilde{G}^{\prime}$ denote the reduction of $\tilde{G}$, and let $W^{\prime}$ denote the set of all nontrivial vertices of $\tilde{G}^{\prime}$. Then there exists a number $n(p)$ such that when $n \geq n(p)$, each of the following holds.

(i) $\left|W^{\prime}\right| \leq p$.

(ii) Every vertex in $J_{p}$ lies in the preimage of a vertex in $W^{\prime}$.

(iii) If $\tilde{G}^{\prime}$ has a $W^{\prime}$-circuit, then $G$ has a dominating circuit.

Proof. Let $G^{\prime \prime}=\tilde{G}^{\prime}$. Note that $G^{\prime \prime}$ is reduced by definition. Let $n^{\prime}=$ $\left|V\left(G^{\prime \prime}\right)\right|$. Let

$$
c=3 p+7
$$

and let $\quad W=\left\{v \in V\left(G^{\prime \prime}\right): d_{G^{\prime \prime}}(v) \leq c\right\}$ and $W^{\prime}=\{v \in W: v$ is nontrivial $\}$.

Claim 1. For any $v \in W^{\prime}$, if $H_{v}$ denotes the preimage of $v$ in $G$, then

$$
\left|V\left(H_{v}\right)\right| \geq \frac{n}{p}-\epsilon+1-d_{G^{\prime \prime}}(v)
$$

Proof. Let $\operatorname{Out}\left(H_{v}\right)=\left\{x \in V\left(H_{v}\right): N_{G}(x) \neq N_{H_{v}}(x)\right\}$ and $\operatorname{In}\left(H_{v}\right)=$ $V\left(H_{v}\right)-\operatorname{Out}\left(H_{v}\right)$. If there is an edge $x y \in E\left(H_{v}\right)$ such that $d(x) \geq d(y)$ and $x \in \operatorname{In}\left(H_{v}\right)$, then by (4.5), $\left|V\left(H_{v}\right)\right| \geq d(x)+1 \geq n / p-\epsilon+1$, and so the Claim holds. Therefore,we assume that for any edge $x y \in E\left(H_{v}\right)$ with $d(x) \geq d(y)$, we always have $x \in \operatorname{Out}\left(H_{v}\right)$. Thus $\left|O u t\left(H_{v}\right)\right| \geq 1$, and so by

$\left|V\left(H_{v}\right)\right|=\left|\operatorname{In}\left(H_{v}\right)\right|+\left|O u t\left(H_{v}\right)\right| \geq 1+d_{G}(x)-d_{G^{\prime \prime}}(v) \geq \frac{n}{p}-\epsilon+1-d_{G^{\prime \prime}}(v)$.

Claim 1 is proved. 
Claim 2. $\left|W^{\prime}\right| \leq p$.

Proof. Since $d_{G^{\prime \prime}}(v) \leq c$ for $v \in W$, and $W^{\prime} \subset W$, by Claim 1, we have $n \geq\left|W^{\prime}\right|(n / p-\epsilon+1-c)$. Thus, $\left|W^{\prime}\right| \leq n p /(n-p(\epsilon+c-1))$. Since $\epsilon$ and $c$ are constants, when $n$ is large (say $n>(p+1) p(\epsilon+c-1)),\left|W^{\prime}\right| \leq p$. This proves Claim 2.

Claim 3. $V\left(G^{\prime \prime}\right)=W$.

Proof. By contradiction, we assume that $V\left(G^{\prime \prime}\right)-W \neq \emptyset$. Note that every vertex in $V\left(G^{\prime \prime}\right)-W$ has degree at least $c+1$ in $G^{\prime \prime}$. Since $G^{\prime \prime}$ is simple and $K_{3}$-free by Theorem $\mathrm{G}$, this means that

$$
n^{\prime}=\left|V\left(G^{\prime \prime}\right)\right| \geq c+2 .
$$

Count the incidences to get $c\left|V\left(G^{\prime \prime}\right)-W\right| \leq 2\left|E\left(G^{\prime \prime}\right)\right| \leq 4 n^{\prime}-8$, which means $\left|V\left(G^{\prime \prime}\right)-W\right| \leq\left(4 n^{\prime}-8\right) / c$. It follows that

$$
|W|=n^{\prime}-\left|V\left(G^{\prime \prime}\right)-W\right| \geq(1-4 / c) n^{\prime}+\frac{8}{c} .
$$

Note that every vertex in $W-W^{\prime}$ has degree at least 3 in $G^{\prime \prime}$, and when $n$ is large, $W-W^{\prime}$ is independent in $G^{\prime \prime}$. By Theorem 4.2.1, by Claim 2, and by $(4.8)$

$$
2 n^{\prime}-4 \geq\left|E\left(G^{\prime \prime}\right)\right| \geq 3\left|W-W^{\prime}\right| \geq(3-12 / c) n^{\prime}+\frac{24}{c}-3 p .
$$

It follows that

$$
3 p-4 \geq\left(1-\frac{12}{c}\right) n^{\prime}+\frac{24}{c} .
$$

By (4.7), $n^{\prime} \geq c+2$. Thus, (4.9) implies that

$$
3 p-4 \geq\left(1-\frac{12}{c}\right)(c+2)+\frac{24}{c}=c-10,
$$


and so by (4.6) $3 p+6 \geq c=3 p+7$, a contradiction. This shows that $V\left(G^{\prime \prime}\right)=W$.

Claim 3 implies that $W^{\prime}$ is the set of all nontrivial vertices of $\tilde{G}^{\prime}$. This shows that Lemma 4.3.1 (i) holds.

Claim 4. Every vertex in $J_{p}(G)$ is contained in the preimage of some vertex in $W^{\prime}$.

Proof. Since $\epsilon$ and $p$ are constants, when $n$ is large enough, the degree of vertices in $J_{p}(G)$ will exceed $c$, and so the Claim follows from Claim 3. This shows that Lemma 4.3.1(ii) holds.

It remains to prove Lemma 4.3.1(iii). By Lemma 4.2.4 and by Lemma 4.3.1(ii), $G$ has a circuit $L$ such that $J_{p} \subseteq V(L)$. Since (4.5) holds for $G, G-V(L)$ must be edgeless, and so $L$ is dominating. The proof is complete.

Lemma 4.3.2 Suppose that $D_{2}(G)$ is an independent set of $G$. If $\kappa^{\prime}(G-$ $\left.\left(D_{1}(G) \cup D_{2}(G)\right)\right) \geq 3$, then $\kappa^{\prime}(\tilde{G}) \geq 3$.

Proof. Let $G_{1,2}=G-\left(D_{1}(G) \cup D_{2}(G)\right)$ and let $D_{2}=\left\{z_{1}, z_{2}, \cdots, z_{s}\right\}$. For each $z_{i}$, let $u_{i}$ and $v_{i}$ be the two neighbors of $z_{i}$, where $1 \leq i \leq s$. Note that $\tilde{G}$ is obtained from $G_{1,2}$ by adding edges $\left\{u_{i} v_{i}: 1 \leq i \leq s\right\}$ and deleting all possibly resulting loops (which may occur when $u_{i}=v_{i}$, for some $i$ ). Since $\kappa^{\prime}\left(G_{1,2}\right) \geq 3$, it follows that $\kappa^{\prime}(\tilde{G}) \geq 3$.

Lemma 4.3.3 If $\kappa^{\prime}(G) \geq 3$ and if $G^{\prime}$ is the reduction of $G$, then $\kappa^{\prime}\left(\tilde{G}^{\prime}\right) \geq 3$. 
Proof Let $X \subseteq E\left(\tilde{G}^{\prime}\right)$ be an edge cut of $\tilde{G}^{\prime}$. Note that by the definition of $\tilde{G}, X$ can be viewed as a subset of $G$. Therefore, $X$ is also an edge cut of $G$. Since $X \subseteq E\left(\tilde{G}^{\prime}\right), X$ does not contain any pendant edges of $G$, and so $X$ cannot consist of the two edges incident with a vertex in $D_{2}(G)$. Therefore, $X$ is an edge cut of $\tilde{G}$. Since it is assumed that $\kappa^{\prime}(G) \geq 3$, one must have $|X| \geq 3$ and so $\kappa^{\prime}\left(\tilde{G}^{\prime}\right) \geq 3$.

We shall prove a slightly stronger version of Theorem 4.1.9 By Lemma 4.3.2. Theorem 4.3.4 below implies Theorem 4.1.9.

Theorem 4.3.4 Let $G$ be a simple graph with $n$ vertices such that $\kappa^{\prime}(\tilde{G}) \geq 3$. If (4.5) holds, then for $n$ large, either $L(G)$ is Hamiltonian, or $G$ has the Petersen graph as a nontrivial contraction.

Proof. Let $G^{\prime}$ denote the reduction of $G$, and let $\tilde{G}^{\prime}$ denote the reduction of $\tilde{G}$. Let $W^{\prime}$ denote the set of nontrivial vertices of $G$. By Lemma 4.3.1(i) with $p=12,\left|W^{\prime}\right| \leq 12$. Since $\kappa^{\prime}(\tilde{G}) \geq 3$, one has $W^{\prime} \cap\left(D_{1}\left(G^{\prime}\right) \cup D_{2}\left(G^{\prime}\right)\right)=\emptyset$. Therefore, $W^{\prime} \subseteq V\left(\tilde{G}^{\prime}\right)$.

Since $\left|W^{\prime}\right| \leq 12$ and since $\kappa^{\prime}\left(\tilde{G}^{\prime}\right) \geq 3$, it follows by Theorem 4.1 .2 that either $\tilde{G}^{\prime}$ has Petersen graph as a nontrivial contraction, or $\tilde{G}^{\prime}$ has a $W^{\prime}$ circuit $H^{\prime}$.

Since $\tilde{G}^{\prime}$ is a contraction of $G^{\prime}$, and $G^{\prime}$ is a contraction of $G$, if the Petersen graph is a nontrivial contraction of $\tilde{G}^{\prime}$, then the Petersen graph is also a nontrivial contraction of $G$, and we are done. Therefore, we assume that $\tilde{G}^{\prime}$ has a $W^{\prime}$-circuit $H^{\prime}$. By Lemma 4.3.1, we conclude that $G$ has a circuit $H$ that contains all vertices of $J_{12}$, and $G$ has a dominating circuit. Therefore by Theorem 4.1.1, $L(G)$ is Hamiltonian. 


\subsection{Proofs for Theorems 4.1.2 and 4.1.3}

We shall argue by contradiction. Some more notations and lemmas are needed.

Let $G$ be a graph and let $v \in V(G)$. Then $N_{G}(v)$ denote the set of vertices in $V(G)$ that are adjacent to $v$ in $G ; E_{G}(v)$ denote the set of edges incident with $v$ in $G$ and for each $i \geq 1$,

$$
D_{i}^{*}(G)=\bigcup_{j \geq i} D_{j}(G) .
$$

For $v \in D_{4}^{*}(G)$, let $N_{G}(v)=\left\{v_{1}, v_{2}, \cdots, v_{d}\right\}$, where $d=d_{G}(v) \geq 4$. For a 4-cycle $C_{4}$, let $V\left(C_{4}\right)=\{x, y, z, w\}$ and let $E\left(C_{4}\right)=\{x y, y z, z w, w x\}$. Let $G_{v}$ be a graph obtained from $G-v$ and $C_{4}$ by joining $x$ to $v_{1}, y$ to $v_{2}, z$ to $v_{3}$, and $w$ to $v_{i}$ for all $i \geq 4$ as shown below.
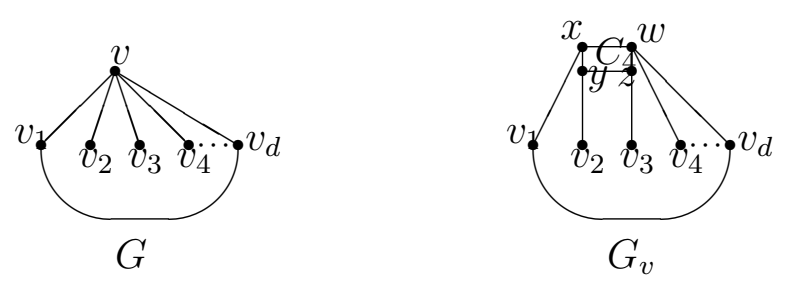

Figure 4.2

Lemma 4.4.1 Let $G$ be a 3-edge-connected graph and let $v \in D_{4}^{*}(G)$. If $v$ is not a cut-vertex, then $G_{v}$ is 3-edge-connected.

Proof. By way of contradiction, suppose that $G_{v}$ has an edge cut $E_{0} \subseteq E(G)$ with $\left|E_{0}\right| \leq 2$. Let $G_{1}$ and $G_{2}$ be the two components of $G_{v}-E_{0}$. Note that if all the vertices of $N_{G}(v)=\left\{v_{1}, v_{2}, \cdots, v_{d}\right\}$ are in the same component (say $G_{1}$ ), then $E_{0}$ is an edge cut of $G$ with $\left|E_{0}\right| \leq 2$, contrary to that $G$ is 
3-edge-connected. Therefore, $E_{0} \subseteq E\left(C_{4}\right)=\{x y, y z, z w, w x\}$ and $\left|E_{0}\right|=2$. However, this implies that $v$ is a cut-vertex, a contradiction. Hence, $G_{v}$ is 3-edge-connected.

Let $S \subseteq V(G)$ be a vertex set, and let $v \in D_{4}^{*}(G)$ and $v^{\prime} \in V\left(C_{4}\right)$. Define

$$
S^{\prime}= \begin{cases}S & \text { if } v \notin S, \\ (S-v) \cup v^{\prime} & \text { otherwise. }\end{cases}
$$

Then $\left|S^{\prime}\right|=|S|$ and $S^{\prime} \subseteq V\left(G_{v}\right)$.

Lemma 4.4.2 Let $G$ be a graph, and let $v \in D_{4}^{*}(G)$. Let $S$ be a vertex subset of $V(G)$, and $S^{\prime}$ be the set defined above. Then each of the following holds:

a. If $G_{v}$ has a circuit $H_{1}$ such that $S^{\prime} \subseteq V\left(H_{1}\right)$, then $G$ has a circuit $H$ such that $S \subseteq V(H)$.

b. If $G_{v}$ can be contracted to the Petersen graph such that the contraction preimage of each vertex in the Petersen graph contains at least one vertex in $S^{\prime}$ then $G$ can be contracted to the Petersen graph such that the contraction preimage of each vertex in the Petersen graph contains at least one vertex in $S$.

Proof. Obvious.

Proof of Theorem 4.1.2. We argue by induction on

$$
f(G)=\sum_{v \in D_{4}^{*}(G)} d_{G}(v) .
$$


If $f(G)=0$, then $G$ is a cubic 3-connected graph, and so the theorem follows from Theorem B. Assume that $f(G)>0$. Then $\left|D_{4}^{*}(G)\right| \geq 1$. Pick $v \in D_{4}^{*}(G)$. If $v$ is not a cut-vertex, then we define $G_{v}$ as shown in Figure 2 . By Lemma 4.4.1 $G_{v}$ is also 3-edge-connected. By the definition of $f(G)$ and $G_{v}$, we have $f\left(G_{v}\right)=f(G)-1$. Let $v^{\prime} \in V\left(C_{4}\right)=\{x, y, z, w\}$. Define

$$
S^{\prime}= \begin{cases}S & \text { if } v \notin S, \\ (S-v) \cup v^{\prime} & \text { otherwise. }\end{cases}
$$

Then $\left|S^{\prime}\right|=|S| \leq 12$ and $S^{\prime} \subseteq V\left(G_{v}\right)$. By induction, either $G_{v}$ has an $S^{\prime}$-circuit $H^{\prime}$, or $G_{v}$ can be contracted to the Petersen graph such that the contraction preimage of each vertex in the Petersen graph contains at least one vertex in $S^{\prime}$. Therefore, Theorem 4.1.2 follows from Lemma 4.4.2.

Next we only need to consider the case that $v$ is a cut-vertex.

Let $H_{1}$ and $H_{2}$ be the two components of $G-v$. Let $G_{1}=G\left[V\left(H_{1}\right) \cup v\right]$ and $G_{2}=G\left[V\left(H_{2}\right) \cup v\right]$. Note that since $G$ is 3-edge-connected, $G_{i}(i=1,2)$ is also 3-edge-connected. Obviously,

$$
f\left(G_{i}\right)<f(G) \quad(i=1,2) .
$$

Let $S_{i}=S \cap V\left(G_{i}\right)(i=1,2)$. We may assume that $\left|S_{2}\right| \leq\left|S_{1}\right|$. If $S_{2}=\emptyset$, then $S \subseteq V\left(G_{1}\right)$. By (4.10) and then by induction, the theorem statement holds for $G_{1}$, and so the theorem holds in this case. Without loss of generality, we assume that $1 \leq\left|S_{2}\right| \leq\left|S_{1}\right| \leq 11$. For $i=1,2$, define

$$
S_{i}^{\prime}= \begin{cases}S_{i} & \text { if } v \in S, \\ S_{i} \cup v & \text { otherwise. }\end{cases}
$$

Then $2 \leq\left|S_{2}^{\prime}\right| \leq\left|S_{1}^{\prime}\right| \leq 12$. By (4.10) and then by induction, we know that either $G_{i}$ has a circuit $H_{i}$ such that $S_{i}^{\prime} \subseteq V\left(H_{i}\right)$ or $G_{i}$ can be contracted to 
the Petersen graph such that the contraction preimage of each vertex in the Petersen graph contains at least one vertex of $S_{i}^{\prime}$. There are two cases to be considered here.

Case 1. $G_{1}$ is contractible to the Petersen graph such that the contraction preimage of each vertex in the Petersen graph contains at least one vertex of $S_{1}^{\prime}$. Since $v \in S_{1}^{\prime}, v$ is in one of the preimage of a vertex in the Petersen graph. Note that $G_{1}=G / G_{2}$. Then $G$ can be contracted to the Petersen graph in such a way that by contracting $G_{2}$ to $v$, and then by successively contracting the preimage of each vertex of the Petersen graph in $G_{1}$. Obviously, The contraction preimage of each vertex in the Petersen graph contains at least one vertex of $S$. The theorem is proved in this case.

Case 2. $G_{1}$ has a circuit $H_{1}$ such that $S_{1}^{\prime} \subseteq V\left(H_{1}\right)$. Since $\left|S_{2}^{\prime}\right| \leq$ $\left|S_{1}^{\prime}\right| \leq 12,\left|S_{2}^{\prime}\right| \leq 6$. By (4.10) and by induction, $G_{2}$ has a circuit $H_{2}$ such that $S_{2}^{\prime} \subseteq V\left(H_{2}\right)$. Since $v \in S_{i}^{\prime} \subseteq V\left(H_{i}\right), H=H_{1} \cup H_{2}$ is a circuit in $G$ with $S \subseteq V(H)=V\left(H_{1}\right) \cup V\left(H_{2}\right)$. The proof is completed.

Remark. Theorem 4.1.3 can be proved by using Theorem 4.1.5 and the same techniques used in the proof of Theorem 4.1.2. 


\section{Chapter 5}

\section{Group chromatic number of some graphs}

\section{$5.1 \quad$ Introduction}

In this chapter, the degree of $v$ in the graph $G$ is denoted by $\operatorname{deg}(v, G)$. Denote $N(v, G)=\{u \in V(G), u v \in E(G)\}$. We let $\chi(G)$ denote the chromatic number of a graph $G$ and $\Delta(G)$ denotes the maximum degree of graph $G$. We use $H \subseteq G$ to denote the fact that $H$ is a subgraph of $G$.

Let $A$ denote an Abelian group and $F(G, A)$ denote the set of all functions from $E(G)$ to $A$. For $f \in F(G, A)$, an $(A, f)$-coloring of $G$ under the orientation $D$ is a function $c: V(G) \mapsto A$ such that for every directed edge $e=u v, c(u)-c(v) \neq f(u v) . G$ is $A$-colorable under the orientation $D$ if

for any function $f \in F(G, A), G$ has an $(A, f)$-coloring. It is known [34] that $A$-colorable is independent of the choice of the orientation. The group 
chromatic number of a graph $G$ is defined to be the minimum $m$ for which $G$ is $A$-colorable for any Abelian group $A$ of order $\geq m$ under a given orientation $D$, and is denoted by $\chi_{g}(G)$.

Let $H \subset G$ be graphs, and $A$ be a group. Given an $f \in F(G, A)$, if for an $\left(A,\left.f\right|_{E(H)}\right)$-coloring $c_{0}$ of $H$, there is an $(A, f)$-coloring $c$ of $G$ such that $c$ is an extension of $c_{0}$, then we say that $c_{0}$ is extended to $c$. If any $\left(A,\left.f\right|_{E(H)}\right)$-colotring $c_{0}$ of $H$ can be extendedtoan $(A, f)$-coloring $c$, then we say that $(G, H)$ is $(A, f)$-extendsible. If for any $f \in F(G, A),(G, H)$ is $(A, f)$-extensible, then $(G, H)$ is A-extensible.

\subsection{The group chromatic number of $K_{3,3}$-minor free graphs}

Jaeger et al [34] proved the following result.

Theorem 5.2.1 (Jeager, Linial, Payan, and Tarsi [34]). If $G$ is a simple planar graph, then $\chi_{g}(G) \leq 6$.

This has been improved by Lai and Zhang.

Theorem 5.2.2 ([39]) If $G$ is a simple graph without a $K_{5}$-minor, then $\chi_{g}(G) \leq 5$. In particular, if $G$ is a simple planar graph, then $\chi_{g}(G) \leq 5$.

Wagner's Theorem is used in our proof of Theorem 5.2.4.

Theorem 5.2.3 (Wagner [55])Suppose that $G$ is not planar with $|V(G)| \geq$ 6. If $G$ is 3-connected,then $G$ contains a $K_{3,3}$ minor. 
We will prove the following result which extends also Jeager et al [34] result.

Theorem 5.2.4 Let $G$ be a connected simple graph without a $K_{3,3}$ minor and let $A$ be a group with $|A| \geq 5$. Suppose that $H$ is a subgraph of $G$ isomorphic to $K_{2}$. Then $(G, H)$ is A-extensible.

Proof. Let $G$ be a connected simple graph without a $K_{3,3}$ minor and let $A$ be a group with $|A| \geq 5$. Suppose that $H$ is a subgraph of $G$ isomorphic to $K_{2}$. Then $(G, H)$ is $A$-extensible.

Proof. Let $f \in F(G, A)$ and $c_{0}$ be an given $\left(A,\left.f\right|_{E(H)}\right)$-coloring. We shall prove by induction on $|V(G)|$ that $c_{0}$ can be extended to an $(A, f)$-coloring of $G$. By Theorem 2, we may assume that $G$ is not planar.

Assume first that $|V(G)|=5$. Since $G$ is not planar, $G \cong K_{5}$. Let $V\left(K_{5}\right)=\left\{v_{1}, v_{2}, v_{3}, v_{4}, v_{5}\right\}$ and assume that the edge $v_{i} v_{j}$ is oriented from $v_{i}$ to $v_{j}$ if $i<j$. Without loss of generality, we assume that $V(H)=\left\{v_{1}, v_{2}\right\}$ and assume that $c_{0}: v_{k} \mapsto a_{k}$ for $k=1,2$. Define $c: V(G) \mapsto A$ by

$$
\left\{\begin{array}{l}
c\left(v_{k}\right)=c_{0}\left(e_{k}\right)=a_{k}, \text { if } k=1,2 \\
c\left(v_{3}\right)=a_{3} \in A-\left\{c\left(v_{1}\right)-f\left(v_{1} v_{3}\right), c\left(v_{2}\right)-f\left(v_{2} v_{3}\right)\right\} \\
c\left(v_{4}\right)=a_{4} \in A-\left\{c\left(v_{1}\right)-f\left(v_{1} v_{4}\right), c\left(v_{2}\right)-f\left(v_{2} v_{4}\right), c\left(v_{3}\right)-f\left(v_{3} v_{4}\right)\right\} \\
c\left(v_{5}\right)=a_{5} \in A-\left\{c\left(v_{1}\right)-f\left(v_{1} v_{5}\right), c\left(v_{2}\right)-f\left(v_{2} v_{5}\right), c\left(v_{3}\right)-f\left(v_{3} v_{5}\right), c\left(v_{4}\right)-f\left(v_{4} v_{5}\right)\right\}
\end{array}\right.
$$

Thus the resulting coloring is an $(A, f)$-coloring and extension for $c_{0}$.

Assume then that $|V(G)| \geq 6$ and that the theorem holds for graphs with smaller values of $|V(G)|$. Let $T$ be a minimum vertex cut of $G$. By Theorem $10,1 \leq|T| \leq 2$. Let $G_{1}$ and $G_{2}$ be two proper subgraphs of $G$ such that $G_{1} \cup G_{2}=G, V\left(G_{1}\right) \cap V\left(G_{2}\right)=T$. Without loss of generality, we assume that 
$H \subset G_{1}$. If $|T|=1$, let $G_{i}^{\prime}=G_{i}$ for $i=1,2$. If $|T|=2$, Let $T=\{u, v\}$. If $u v \in E$, let $G_{i}^{\prime}=G_{i}$ for $i=1,2$. If $u v \notin E$, Let $G_{i}^{\prime}=G_{i}+u v$ for $i=1,2$. It follows that $G_{1}^{\prime}$ and $G_{2}^{\prime}$ are $K_{3,3}$ free. Since $\left|V\left(G_{1}^{\prime}\right)\right|<|V(G)|$ and $\left|V\left(G_{2}^{\prime}\right)\right|<$ $|V(G)|$. By induction there is an $\left(A,\left.f^{\prime}\right|_{E\left(G_{1}^{\prime}\right)}\right)$-coloring $c_{1}: V\left(G_{1}^{\prime}\right) \mapsto A$ which extends $c_{0}$ and an $\left(A,\left.f^{\prime}\right|_{E\left(G_{2}^{\prime}\right)}\right)$-coloring $c_{2}: V\left(G_{2}^{\prime}\right) \mapsto A$ which extends $C_{1} \mid T$. It follows that an $(A, f)$-coloring $c: V(G) \mapsto A$ extends $c_{0}$.

From Theorem 5.2.4, we easy have the following corollary. As $K_{5}$ does not have a $K_{3,3}$-minor, this result is best possible.

Corollary 5.2.5 Let $G$ be a simple graph without $K_{3,3}$ minors. Then $\chi_{g}(G) \leq$ 5 .

\subsection{The group chromatic number for join of two graphs}

Let $G_{1}$ and $G_{2}$ be subgraphs of $G$. The union $G_{1} \cup G_{2}$ of $G_{1}$ and $G_{2}$ is the subgraph with vertex set $V\left(G_{1}\right) \cup V\left(G_{2}\right)$ and edge set $E\left(G_{1}\right) \cup E\left(G_{2}\right)$. If $G_{1}$ and $G_{2}$ are disjoint, denote the union by $G_{1}+G_{2}$. The join $G \vee H$ of disjoint graphs $G$ and $H$ is the graph obtained from $G+H$ by joining each vertex of $G$ to each vertex of $H$. Let $G$ and $H$ be two given graphs. In proper coloring, it is well known that $\chi(G \vee H)=\chi(G)+\chi(H)$, but it is defferent in the group coloring. It is easy to see that $\chi\left(K_{2,2}\right)=2$ and $K_{2,2}=\overline{K_{2}} \vee \overline{K_{2}}$. Lai and Zhang [38] showed that $\chi_{g}\left(K_{2,2}\right)=3$. We have the following result.

Theorem 5.3.1 (1) Suppose that $G$ and $H$ are two given graphs. Then

$$
\chi_{g}(G+H)=\max \left\{\chi_{g}(G), \chi_{g}(H)\right\} .
$$


(2) Let $H_{1}$ and $H_{2}$ be two subgraphs of $G$ such that $V\left(H_{1}\right) \cap V\left(H_{2}\right)=\emptyset$ and $V(G)=V\left(H_{1}\right) \cup V\left(H_{2}\right)$. Then

$\chi_{g}(G) \leq \min \left\{\max \left\{\chi_{g}\left(H_{1}\right), \max _{v \in V\left(H_{2}\right)} \operatorname{deg}\left(v, H_{1}\right)\right\}+\Delta\left(H_{2}\right)+1\right.$, $\left.\max \left\{\chi_{g}\left(H_{2}\right), \max _{u \in V\left(H_{1}\right)} \operatorname{deg}\left(u, H_{2}\right)\right\}+\Delta\left(H_{1}\right)+1\right\}$.

Proof. (1) Obvously.

(2) Let $k=\max \left\{\chi_{g}\left(H_{1}\right), \max _{v \in V\left(H_{2}\right)} \operatorname{deg}\left(v, H_{1}\right)\right\}+\Delta\left(H_{2}\right)+1$. By symetry we only show that $\chi_{g}(G) \leq k$.

We argue by contradiction and assume that $G$ is a counterexample with $\left|V\left(H_{2}\right)\right|$ minimized. It is easy to see that the theorem holds if $\left|V\left(H_{2}\right)\right|=0$ and so we assume that $\left|V\left(H_{2}\right)\right|>0$. Let $A$ be an Abelian group with $|A| \geq k$ and let $w \in V\left(H_{2}\right)$ such that $\operatorname{deg}(w, G)=\delta\left(H_{2}\right)$. We have

$$
\max \left\{\chi_{g}\left(H_{1}\right), \max _{v \in V\left(H_{2}\right)-w} \operatorname{deg}\left(v, H_{1}\right)\right\}+\Delta\left(H_{2}-w\right)+1 \leq k .
$$

By the choice of $H_{2}, G-w=<V\left(H_{1}\right) \cup V\left(H_{2}-w\right)>$ is $A$-colorable if $|A| \geq k$. Therefore for any $f: E(G-w) \mapsto A$, there is $c: V\left(H_{1}\right) \cup V\left(H_{2}-w\right) \mapsto A$ such that $c(x)-c(y) \neq f(x y)$ for any directed edge $x y$. Without loss of generality we assume that $G$ is oriented such that all the edges incident with $w$ is oriented from $w$. It follows that

$$
\begin{aligned}
\operatorname{deg}(w, G) & =\operatorname{deg}\left(w, H_{1}\right)+\operatorname{deg}\left(w, H_{2}\right) \\
& \leq \max _{v \in V\left(H_{2}\right)} \operatorname{deg}\left(v, H_{1}\right)+\Delta\left(H_{2}\right) \\
& \leq k-1 .
\end{aligned}
$$

We may assume that $N(w, G)=\left\{w_{1}, w_{2}, \ldots, w_{t}\right\}, t \leq k-1$. Since $|A| \geq$ $k$, there is an $a \in A-\cup_{i=1}^{t}\left\{c\left(w_{i}\right)+f\left(w w_{i}\right)\right\}$. Therefore we extend $c$ to 
$c_{1}: V(G) \mapsto A$ by

$$
c_{1}(u)=\left\{\begin{array}{l}
c(u) \text { if } u \in V(G)-w \\
a \text { if } u=w
\end{array}\right.
$$

$c_{1}(u)=c(u)$ if $u \in V(G)-w ; c_{1}(u)=a$ if $w=u$.

Thereforwe $G$ is $A$-colorable if $|A| \geq k$. Thus we get a contradiction and the proof completes.

The bound of Theorem 5.4.2 is sharp. There are infinite examples. Let $G=\overline{K_{2}} \vee \overline{K_{2}}$. Then $\chi(G)=\chi\left(\overline{K_{2}}\right)+\chi\left(\overline{K_{2}}\right)$ and $\chi_{g}(G)=\min \left\{\max \left\{\chi_{g}\left(\overline{K_{2}}\right)\right.\right.$, $\left.\max _{v \in V\left(\overline{K_{2}}\right)} \operatorname{deg}\left(v, \overline{K_{2}}\right)\right\}+\Delta\left(\overline{K_{2}}\right)+1, \max \left\{\chi_{g}\left(\overline{K_{2}}\right), \max _{u \in V\left(\overline{K_{2}}\right)} \operatorname{deg}\left(u, \overline{K_{2}}\right)\right\}$ $\left.+\Delta\left(\overline{K_{2}}\right)+1\right\}=\min \{\max \{1,2\}+1, \max \{1,2\}+1\}=3$. In fact when $m \geq n \geq$ $2, G=K_{n} \vee K_{m}$ is another such example. $\chi_{g}(G)=\min \left\{\max \left\{\chi_{g}\left(K_{n}\right),\left|V\left(K_{n}\right)\right|\right\}+\right.$ $\left.\Delta\left(K_{m}\right)+1, \max \left\{\chi_{g}\left(K_{m}\right),\left|V\left(K_{m}\right)\right|\right\}+\Delta\left(K_{n}\right)+1\right\}=\min \{\max \{n+(m-1)+$ $1, m+(n-1)+1\}=m+n$.

From Theorem 5.4.2, we obtain the following corollary.

Corollary 5.3.2 Suppose that $G$ and $H$ are two graphs. Then

$$
\chi_{g}(G \vee H) \leq \min \left\{\left|V\left(H_{1}\right)\right|+\Delta\left(H_{2}\right)+1,\left|V\left(H_{2}\right)\right|+\Delta\left(H_{1}\right)+1\right\} .
$$

\subsection{The group chromatic number for the $k^{t h}$ power of a graph}

Lai and Zhang [38] proved the analogue of Brook's Theorem [10] 
Theorem 5.4.1 (Lai and Zhang [38]) For any connected simple graph $G$,

$$
\chi_{g}(G) \leq \Delta(G)+1
$$

with equality if and if either $\Delta(G)=2$ and $G$ is a cycle; or $\Delta(G) \geq 3$ and $G$ is complete.

Let $k$ be any integer and let $G^{k}$ denote the $k t h$ power of $G$ : i.e., the graph with $V\left(G^{k}\right)=V(G)$, where $u v \in E\left(G^{k}\right)$ if and only if $u$ and $v$ lie at distance at most $k$ in $G$.

We obtain the following result which extends Theorem 5.4.1.

Theorem 5.4.2 Let $k$ be an integer. If $G$ is a connected graph, then

$$
\chi_{g}\left(G^{k}\right) \leq \Delta^{k}+1
$$

and equality holds if and only if either $G=K_{2}$ or $G$ is a $\Delta$-regular graph of girth $2 k+1$ and order $\Delta^{k}+1$.

Remark When $k=2$, Hoffman and Singleton [30] have show that the only $\Delta$-regular graph of girth 5 and order $\Delta^{2}+1$ are the five cycle, the Petersen graph, the Hoffman-Singleton graph $(\Delta=7)$, and possible such a graph with $\Delta=57$.

Proof of Theorem 5.4.2. Let $G$ be connected. For any $v \in V(G)$, we have

$$
\operatorname{deg}\left(v, G^{k}\right) \leq \Delta(G)^{k}
$$

with equality if and only if $v$ does not lie in any $l$-cycle for $3 \leq l \leq 2 k$ and $v$ and all vertices at distance at most $k-1$ from $v$ have degree $\Delta$. Hence

$$
\Delta\left(G^{k}\right) \leq \Delta(G)^{k} .
$$


By Theorem 8, we have

$$
\chi_{g}\left(G^{k}\right) \leq \Delta(G)^{k}+1
$$

with equality if and only if $G^{k}$ is complete with $\Delta\left(G^{k}\right)+1$ vertices. By (3) and (4), (1) holds. All that remains is to consider the case where equality holds in (1).

suppose that (1) holds with equality. Then equality holds in (3) and (4). $G^{k}$ is complete with $\Delta\left(G^{k}\right)+1$ vertices, and for any vertex $v$ such that $\operatorname{deg}(v, G)=\Delta$, the equality in (3) implies eqality in (2). Therefore, any vertex $v$ of degree $\Delta$ does not lie in any $l$-cycle $3 \leq l \leq 2 k$, and all vertices at distance at most $k$ from $v$ have degree $\Delta$ also. Hence $G$ is regular and $V(G-v)$ consists only of $\Delta$ neighbors of $v$ and exactly $\Delta\left(G^{2}\right)-\Delta$ vertices at distance 2 from $v$, exactly $\Delta\left(G^{3}\right)-\left(\Delta\left(G^{2}\right)-\Delta\right)$ vertices at distance 3 from $v, \ldots$ exactly $\Delta\left(G^{l}\right)-\Delta\left(G^{(l-1)}\right)+\ldots,(-1)^{l} \Delta$ vertices at distance $l, 3 \leq l \leq 2 k$ from $v$, for if some vertex $w \in V(G)$ were at distance $2 k+1$ from, then $v w \notin E\left(G^{k}\right)$, contrary to the completeness of $G^{k}$. It follows that $G$ has order $\Delta^{k}+1$, and $G$ has no $l$-cycle for $3 \leq l \leq 2 k$. Therefore, either $G=K_{2}$ or $G$ has girth $2 k+1$, and $G$ is a $\Delta$-regular graph of order $\Delta^{k}+1$.

Set $f=0$ for and $f \in F(G, A)$ and we have following corollary.

Corollary 5.4.3 Let $k$ be an integer. If $G$ is a connected graph, then

$$
\chi\left(G^{k}\right) \leq \Delta^{k}+1
$$

and equality holds if and only if either $G=K_{2}$ or $G$ is a $\Delta$-regular graph of girth $2 k+1$ and order $\Delta^{k}+1$. 


\section{Chapter 6}

\section{Group chromatic number of planar graphs with girth at least 4}

\subsection{Introduction}

Let $A$ denote an Abelian group and $F(G, A)$ denote the set of all functions from $E(G)$ to $A$. For $f \in F(G, A)$, an $(A, f)$-coloring of $G$ under the orientation $D$ is a function $c: V(G) \mapsto A$ such that for every directed edge $e=u v, c(u)-c(v) \neq f(u v)$. $G$ is $A$-colorable under the orientation $D$ if for any function $f \in F(G, A), G$ has an $(A, f)$-coloring. It is known ([34]) that $A$-colorablity is independent of the choice of the orientation. The group chromatic number of a graph $G$ is defined to be the smallest positive integer $m$ for which $G$ is $A$-colorable for any Abelian group $A$ of order $\geq m$ under a given orientation $D$, and is denoted by $\chi_{g}(G)$. Let $G$ and $H$ be two graphs. 
$G$ is contractible to $H$ if $H$ can be obtained from $G$ by contracting some edges of $G$ and deleting the resulting loops. If $G$ contains a subgraph which is contratible to $\Gamma$, then $\Gamma$ is a minor of $G$. A set of subgraphs of $G$ is said to be independent if no two of them have common vertex.

Our terminology is standard as in [8] except otherwise defined. A $k$ path (k-cycle) denotes a path (cycle) of length $k$. The distance of 4-cycle $v_{1} v_{2} v_{3} v_{4} v_{1}$ and 5-cycle $u_{1} u_{2} u_{3} u_{4} u_{5} u_{1}$ is $\min \left\{d_{G}\left(v_{i}, u_{j}\right) \mid 1 \leq i \leq 4,1 \leq j \leq 5\right\}$, where $d_{G}(u, v)$ denote the length of a shortest $(u, v)$-path in $G$. The girth of graph $G$ is the length of a shortest cycle of $G$. For a plane graph the unique unbounded face is called the outer face. If $C$ is a cycle in a plane graph, then $\operatorname{int}(C)$ is the set of vertices and edges inside $C$. If $\operatorname{int}(\mathrm{C})=\emptyset$, then $C$ is facial. If the outer face is bounded by a cycle, we call it the outer cycle. A separating cycle is a cycle $C$ such that the graph has at least one vertex outside $C$ and at least one vertex inside $C$. Thoughout this chapter, $Z_{3}$ denote the 3 element field as well as the 3 element group.

Jaeger, Linial, Payan and Tarsi [34] proved that if $G$ is a planar graph, then $\chi_{g}(G) \leq 6$. It is shown (see [38]) that if $G$ is a graph without a $K_{5}$-minor or without a $K_{3,3}$-minor, then $\chi_{g}(G) \leq 5$. Jaeger, Linial, Payan and Tarsi [34] also proved that if $G$ is a planar graph with girth $\geq 4$, then $\chi_{g}(G) \leq 4$. In this chapter we prove the following results.

Theorem 6.1.1 Suppose that $G$ is a planar graph with girth 4 such that all 4-cycles are independent and every 4-cycle is facial. If the minimum distance between 4-cycles and 5-cycles is at least 1 , then $\chi_{g}(G) \leq 3$.

Theorem 6.1.2 If $G$ is a $K_{3,3}$-minor free graph with girth at least 5 , then $\chi_{g}(G) \leq 3$. 
The proofs of these results are in Section 6.2 and Section 6.3, respectively.

For a vertex $v \in V(G)$, let

$$
E^{-}(v)=\{(u, v) \in E(G): u \in V(G)\}, E^{+}(v)=\{(v, u) \in E(G): u \in
$$
$V(G)\}$ and $E(v)=E^{+}(v) \cup E^{-}(v)$.

Throughout this paper $A$ denotes a nontrivial Abelian group and let $A^{*}=$ $A-\{0\}$. Define

$$
F^{*}(G, A)=\left\{f: E(G) \mapsto A^{*}\right\}
$$

For each $f \in F(G, A)$, the boundary of $f$ is a function $\partial f: V(G) \mapsto A$ defined by

$$
\partial f(v)=\sum_{e \in E^{+}(v)} f(e)-\sum_{e \in E^{-}(v)} f(e),
$$

where " $\sum$ " refers to the addition in $A$. Denote

$$
Z(G, A)=\left\{b: V(G) \mapsto A \text { such that } \sum_{v \in V(G)} b(v)=0\right\} .
$$

A graph $G$ is $A$-connected if $G$ has an orientation $D$ such that for every function $b \in Z(G, A)$ there is a function $f \in F^{*}(G, A)$ such that $b=\partial f$.

Jaeger, Linial, Payan and Tarsi [34] had the following conjecture.

Conjecture 6.1.3 Every 5-edge connected graph is $Z_{3}$-connected.

Let $G$ be a connected plane graph, $G^{*}$ the geometric dual of $G$, and $A$ an Abelian group. Jeager et al [34] showed that $G$ is $A$-connected if and only if $G^{*}$ is $A$-colorable. By Theorem 6.1.1, we comfirm this conjecture for planar graphs.

Corollary 6.1.4 Every 5-edge connected planar graph is $Z_{3}$-connected. 


\section{2 $\quad Z_{3}$-Coloring Theorem}

Let $\mathcal{F}$ denote the set of connected graphs such that a graph $G \in \mathcal{F}$ if and only if each of the following holds:

(A1) $G$ is a planar with girth at least 4 and every 4-cycle is facial;

(A2) all 4-cycles are independent;

(A3) the minimum distance between 4-cycles and 5-cycles is at least 1.

In the discussions below, when we assume that $G \in \mathcal{F}$, we also assume that $G$ is embedded in the plane with an orientation.

Theorem 6.2.1 Suppose $G \in \mathcal{F}$ and let $f \in F\left(G, Z_{3}\right)$. Let $W$ be a set of vertices on the outer cycle in $G$ such that

(W1) either $G[W]$ is edgeless or

(W2) $G[W]$ has exactly one edge $e=x y$ and $G$ has no 2-path from $x$ to another vertex in $W$.

Assume that each vertex $w \in W$ is associated with a $b_{w} \in Z_{3}$, that $u, v \notin$ $W$ are two adjacent vertices on the out cycle of $G$ ( assume that uv is oriented from $u$ to $v)$, that $G[\{u, v, x, y\}]$ does not contain a 4-cycle if $x y$ is an edge of $G[W]$, and that $a_{u}, a_{v} \in Z_{3}$ with $a_{u}-a_{v} \neq f(u v)$. Define $c_{1}:\{u, v\} \mapsto Z_{3}$ by $c_{1}(u)=a_{u}, c_{1}(v)=a_{v}$. Then $c_{1}$ can be extended to $c: V(G) \mapsto Z_{3}$ such that $\left.c\right|_{\{u, v\}}=c_{1}$ and

(i) $c(w) \neq b_{w}$ for every vertex $w \in W$,

(ii) $c\left(x^{\prime}\right)-c\left(y^{\prime}\right) \neq f\left(x^{\prime} y^{\prime}\right)$ for any edge $x^{\prime} y^{\prime} \in E$ oriented from $x^{\prime}$ to $y^{\prime}$.

Remark. The condition that $G[\{u, v, x, y\}]$ does not contain a 4-cycle if $x y$ is an edge of $G[W]$ can not be relaxed. 
Let $C=x_{1} x_{2} x_{3} x_{4} x_{1}$ be a 4-cycle. Assume that $W=\left\{x_{3}, x_{4}\right\}$ and $b_{x_{3}}=$ $1, b_{x_{4}}=1$, and $C$ is oriented from $x_{i}$ to $x_{i+1}, 1 \leq i \leq 3$ and from $x_{4}$ to $x_{1}$.

Define $f \in F\left(C, Z_{3}\right)$ as follows: $f(e)=0$ if $e \in E(C)-\left\{x_{4} x_{1}\right\}$ and $f\left(x_{4} x_{1}\right)=-1$. Define $c_{1}:\left\{x_{1}, x_{2}\right\} \mapsto Z_{3}$ by $c_{1}\left(x_{1}\right)=1, c_{1}\left(x_{2}\right)=0$. Then $c_{1}$ can not be extended to $c: V(C) \mapsto Z_{3}$ such that $\left.c\right|_{\left\{x_{1}, x_{2}\right\}}=c_{1}$.

We need some preparations before presenting the proof of Theorem 6.2.1.

Lemma 6.2.2 Let $G \in \mathcal{F}$ and let $C: x_{1} x_{2} \ldots x_{5} x_{1}$ be a 5-cycle. Assume that $f \in F\left(G, Z_{3}\right)$ and $x_{i} x_{i+1}$ is oriented from $x_{i}$ to $x_{i+1}, 1 \leq i \leq 5$ (indices mod 5). For each map $c_{1}: V(C) \mapsto Z_{3}$, there is some $i \in\{1,2, \ldots 5\}$ (indices mod 5) such that

$$
c_{1}\left(x_{i}\right)-f\left(x_{i} x_{i+1}\right) \neq c_{1}\left(x_{i+2}\right)+f\left(x_{i+1} x_{i+2}\right) .
$$

Proof.. By contradiction, suppose that

$$
c_{1}\left(x_{i}\right)-f\left(x_{i} x_{i+1}\right)=c_{1}\left(x_{i+2}\right)+f\left(x_{i+1} x_{i+2}\right)
$$

for every $1 \leq i \leq 5$ (indices mod 5$)$. Since $Z_{3}$ is a field, by (1) we have

$$
f\left(x_{1} x_{2}\right)+f\left(x_{2} x_{3}\right)+\ldots+f\left(x_{k} x_{1}\right)=0 .
$$

Thus we have

$$
\begin{aligned}
c_{1}\left(x_{1}\right) & =f\left(x_{1} x_{2}\right)+c_{1}\left(x_{3}\right)+f\left(x_{2} x_{3}\right) \\
& =f\left(x_{3} x_{4}\right)+c_{1}\left(x_{5}\right)+f\left(x_{4} x_{5}\right)+f\left(x_{2} x_{3}\right)+f\left(x_{1} x_{2}\right) \\
& =-f\left(x_{5} x_{1}\right)+c_{1}\left(x_{5}\right) .
\end{aligned}
$$

It follows that $c_{1}\left(x_{5}\right)-c_{1}\left(x_{1}\right)=f\left(x_{5} x_{1}\right)$, a contradiction. 
Theorem 6.2.1 implies the following Corollary 6.2.3. We shall argue by induction on $|V(G)|$ to prove Theorem 6.2.1. Our induction hypothesis will assume the truth of both Theorem 6.2.1 and Corollary 6.2.3 for smaller values of $|V(G)|$ to prove Theorem 6.2.1 for the current value of $|V(G)|$.

Corollary 6.2.3 Let $G \in \mathcal{F}$ with outer cycle $C: x_{1} x_{2} \ldots x_{5} x_{1}$ and let $f \in$ $F\left(Z_{3}, G\right)$. If $c_{1}: V(C) \mapsto Z_{3}$ is a $\left(Z_{3}, f\right)$-coloring, then $c_{1}$ can be extended to $c, a\left(Z_{3}, f\right)$-coloring of $G$, such that $\left.c\right|_{V(C)}=c_{1}$.

Proof. Assume that $C$ is oriented from $x_{i}$ to $x_{i+1}, 1 \leq i \leq 5$ (indices mod $5)$.

By Lemma 6.2.2, we assume that $c_{1}\left(x_{5}\right)+f\left(x_{4} x_{5}\right) \neq c_{1}\left(x_{3}\right)-f\left(x_{3} x_{4}\right)$. Let $W=\left\{x_{3}, x_{5}\right\}$. Since $c_{1}$ is a $\left(Z_{3}, f\right)$-coloring, $c_{1}\left(x_{2}\right)-c_{1}\left(x_{3}\right) \neq f\left(x_{2} x_{3}\right)$ and $c_{1}\left(x_{5}\right)-c_{1}\left(x_{1}\right) \neq f\left(x_{5} x_{1}\right)$. We pick $b_{x_{3}} \in Z_{3}-\left\{c_{1}\left(x_{2}\right)-f\left(x_{2} x_{3}\right), c_{1}\left(x_{3}\right)\right\}, b_{x_{5}} \in$ $Z_{3}-\left\{c_{1}\left(x_{1}\right)+f\left(x_{1} x_{5}\right), c_{1}\left(x_{5}\right)\right\}$. By Theorem 6.2.1, $c_{1}:\left\{x_{1}, x_{2}\right\} \mapsto Z_{3}$ can be extended to $c: V(G) \mapsto Z_{3}$ such that $\left.c\right|_{\left\{x_{1}, x_{2}\right\}}=c_{1}$ and $c(w) \neq b_{w}$ for every $w \in W$.

By the choice of $b_{x_{3}}$ and $b_{x_{5}}, c\left(x_{3}\right) \in\left\{c_{1}\left(x_{2}\right)-f\left(x_{2} x_{3}\right), c_{1}\left(x_{3}\right)\right\}, c\left(x_{5}\right) \in$ $\left\{c_{1}\left(x_{1}\right)+f\left(x_{1} x_{5}\right), c_{1}\left(x_{5}\right)\right\}$. Hence $c\left(x_{3}\right)=c_{1}\left(x_{3}\right), c\left(x_{5}\right)=c_{1}\left(x_{5}\right)$.

Since $c\left(x_{4}\right)-c\left(x_{5}\right) \neq f\left(x_{4} x_{5}\right)$ and $c\left(x_{3}\right)-c\left(x_{4}\right) \neq f\left(x_{3} x_{4}\right)$, we have $c\left(x_{4}\right) \in$ $Z_{3}-\left\{c\left(x_{5}\right)+f\left(x_{4} x_{5}\right), c\left(x_{3}\right)-f\left(x_{3} x_{4}\right)\right\}=Z_{3}-\left\{c_{1}\left(x_{5}\right)+f\left(x_{4} x_{5}\right), c_{1}\left(x_{3}\right)-\right.$ $\left.f\left(x_{3} x_{4}\right)\right\}$. Since $c_{1}: V(C) \mapsto Z_{3}$ is a $\left(Z_{3}, f\right)$ coloring, $c_{1}\left(x_{4}\right) \in Z_{3}-\left\{c_{1}\left(x_{5}\right)+\right.$ $\left.f\left(x_{4} x_{5}\right), c_{1}\left(x_{3}\right)-f\left(x_{3} x_{4}\right)\right\}$. Thus $c_{1}\left(x_{4}\right)=c\left(x_{4}\right)$.

In order to prove Theorem 6.2.1, we first prove some lemmas. The following lemmas have the same hypotheses of Theorem 6.2.1 with an additional assumption that 
$G$ is a counterexample to Theorem 6.2.1 and $|V(G)|$ is minimized.

Since $c_{1}$ can be easily extended to a $\left(Z_{3}, f\right)$-coloring for every forest and a 4-cycle which satisfy the condition of Theorem 6.2.1, we may assume that $|V(G)| \geq 5$

Lemma 6.2.4 $\kappa(G) \geq 2$. If $z \in V(G)-W$ and $z \notin\{u, v\}$, then $d_{G}(z) \geq 3$.

Proof. If $G$ is not 2-connected, then $G$ has a block $B$ containing the edge $u v$. By the minimality of $G, c_{1}$ can be extended to a $\left(Z_{3}, f\right)$-coloring of $B$. Let $B_{1}$ be the block which has a common vertex $w_{1}$ with $B$ and pick its adjacent vertex $w_{2}$ in the outer cycle of $B_{1}$. Assume that the edge $w_{1} w_{2}$ is oriented from $w_{1}$ to $w_{2}$ and put $c_{1}\left(w_{2}\right) \in Z_{3}$ such that $c_{1}\left(w_{1}\right)-c_{1}\left(w_{2}\right) \neq$ $f\left(w_{1} w_{2}\right)$. Then $c_{1}$ can be extended to a $\left(Z_{3}, f\right)$-coloring of $B_{1}$, and so on. This contradicts to (3).

By contradiction, suppose that there is a $z_{0} \in V(G)-W$ such that $z_{0} \notin\{u, v\}$ and $d_{G}\left(z_{0}\right)=2$. Let $G_{1}=G-z_{0}$. Denote $N\left(z_{0}\right)=\left\{z_{1}, z_{2}\right\}$ and assume that the edge $z_{0} z_{1}$ is oriented from $z_{0}$ to $z_{1}$ and the edge $z_{0} z_{2}$ is oriented from $z_{0}$ to $z_{1}$. By the minimality of $G, c_{1}$ can be extended to a $\left(Z_{3}, f\right)$-coloring $c_{2}$ of $G_{1}$. Define $c: V(G) \mapsto Z_{3}$ by $c(z)= \begin{cases}c_{2}(z), & \text { if } z \in V(G)-\left\{z_{0}\right\} \\ a \in Z_{3}-\left\{c\left(z_{1}\right)+f\left(z_{0} z_{1}\right), c\left(z_{2}\right)+f\left(z_{0} z_{2}\right)\right\}, & \text { if } z=z_{0}\end{cases}$ Then $c$ is a required $\left(Z_{3}, f\right)$-coloring of $G$, violating to $(3)$.

We assume that $C: x_{1} x_{2} \ldots x_{m} x_{1}$ is the outer cycle of $G$ oriented from $x_{i}$ to $x_{i+1}, 1 \leq i \leq m$ (indices $\bmod m$ ) and for every $z \in N\left(x_{j}\right)-V(C)$, the edge $x_{j} z$ is oriented from $x_{j}$ to $z$. Let $u=x_{1}$ and $v=x_{2}$. If $G[W]$ has an edge $x y$, we can assume that $y=x_{i}, x=x_{i+1}$, where $3 \leq i \leq m-1$. 
Lemma 6.2.5 $C$ has no chord $u^{\prime} v^{\prime}$ for which $G\left[\left\{u^{\prime}, v^{\prime}, x, y\right\}\right]$ is not a 4-cycle.

Proof. Let $C_{1}$ be the cycle in $C \cup\left\{u^{\prime} v^{\prime}\right\}$ containing $u^{\prime} v^{\prime}$ and $u v$. Let $C_{2}$ be the cycle in $C \cup\left\{u^{\prime} v^{\prime}\right\}$ containing $u^{\prime} v^{\prime}$ but not $u v$. Then both $C_{1} \cup \operatorname{int}\left(C_{1}\right) \in \mathcal{F}$ and $C_{2} \cup \operatorname{int}\left(C_{2}\right) \in \mathcal{F}$. By the minimality of $G$ we can extend $c_{1}$ to a $\left(Z_{3}, f\right)$ coloring of $C_{1} \cup \operatorname{int}\left(C_{1}\right)$. Then the coloring of $u^{\prime}$ and $v^{\prime}$ can be extended to a $\left(Z_{3}, f\right)$-coloring of $C_{2} \cup \operatorname{int}\left(C_{2}\right)$, violating to (3).

Throughout the proof of Theorem 6.2.1, we need to redefine $W^{\prime}$ to replace $W$ in order to apply induction hypothesis. In each occassion we induce a set $W^{\prime}$, we only indicate how to assign the new value $b_{z}$ for some $z \in W^{\prime}$ including all $z \in W^{\prime}-W$, while leaving $b_{z}$ unchanged for those $z \in W \cap W^{\prime}$ where $b_{z}$ is not redefined.

Lemma 6.2.6 For $v^{\prime} \in V(G)-V(C), u^{\prime} \in V(C)$ and $w \in W, G$ has no path $u^{\prime} v^{\prime} w$ which satisfies one of the following

(1) $w \notin\{x, y\}$ or

(2) if $w \in\{x, y\}, G\left[\left\{u^{\prime}, v^{\prime}, x, y\right\}\right]$ is not a 4 -cycle.

Proof. If $u^{\prime} v^{\prime} w$ exists, then we define $C_{1}$ and $C_{2}$ as follows: Let $C_{1}$ be the cycle in $C \cup\left\{u^{\prime} v^{\prime}, v^{\prime} w\right\}$ containing $u^{\prime} v^{\prime}, v^{\prime} w$ and $u v$ and let $C_{2}$ be the cycle in $C \cup\left\{u^{\prime} v^{\prime}, v^{\prime} w\right\}$ containing $u^{\prime} v^{\prime}, v^{\prime} w$ but not $u v$. By the minimality of $G, c_{1}$ can be extended to a $\left(Z_{3}, f\right)$-coloring $c_{2}$ of $C_{1} \cup \operatorname{int}\left(C_{1}\right)$. We recall that the edge $u^{\prime} v^{\prime}$ is oriented from $u^{\prime}$ to $v^{\prime}$ and that the edge $v^{\prime} w$ is oriented from $w$ to $v^{\prime}$.

Since $c_{2}$ is a $\left(Z_{3}, f\right)$-coloring of $C_{1} \cup \operatorname{int}\left(C_{1}\right)$ and $c_{2}(w) \neq b_{w}$, we redefine $b_{w} \in Z_{3}-\left\{c_{2}(w), c_{2}\left(v^{\prime}\right)+f\left(v^{\prime} w\right)\right\}$. By the minimality of $G,\left.c_{2}\right|_{\left\{u^{\prime}, v^{\prime}\right\}}$ can be 
extended to a $\left(Z_{3}, f\right)$-coloring $c_{3}$ of $C_{1} \cup \operatorname{int}\left(C_{2}\right)$ such that $\left.c_{3}\right|_{\left\{u^{\prime}, v^{\prime}\right\}}=c_{2}$. It follows that $c_{3}(w)=c_{2}(w)$. Thus we obtain a required $\left(Z_{3}, f\right)$-coloring of $G$, contrary to $(3)$.

Lemma 6.2.7 $G$ does not have 3-path $w_{1} u^{\prime} v^{\prime} w_{2}$ with $w_{1}, w_{2} \in W$ and $u^{\prime}, v^{\prime} \in$ $V(G)-W$ unless $\left\{w_{1}, w_{2}\right\}=\{x, y\}$ and $G\left[\left\{w_{1}, w_{2}, u^{\prime}, v^{\prime}\right\}\right]$ is a 4 -cycle.

Proof. By Lemma 6.2.6, assume that $u^{\prime}, v^{\prime} \in V(G)-V(C)$ and that $G$ has such a 3 -path $w_{1} u^{\prime} v^{\prime} w_{2}$ with $w_{1}, w_{2} \in W$. Assume that the path $w_{1} u^{\prime} v^{\prime} w_{2}$ is oriented by from $w_{1}$ to $u^{\prime}$, from $u^{\prime}$ to $v^{\prime}$ and from $w_{2}$ to $v^{\prime}$. Define $C_{1}$ and $C_{2}$ as follows: Let $C_{1}$ be the cycle in $C \cup\left\{w_{1} u^{\prime}, u^{\prime} v^{\prime}, v^{\prime} w_{2}\right\}$ containing $w_{1} u^{\prime}, u^{\prime} v^{\prime}, v^{\prime} w_{2}$ and $u v$ and let $C_{2}$ be the cycle in $C \cup\left\{w_{1} u^{\prime}, u^{\prime} v^{\prime}, v^{\prime} w_{2}\right\}$ containing $w_{1} u^{\prime}, u^{\prime} v^{\prime}$ and $v^{\prime} w_{2}$ but not $u v$. By the minimality of $G, c_{1}:\{u, v\} \mapsto Z_{3}$ can be extended to a $\left(Z_{3}, f\right)$-coloring $c_{2}$ of $C_{1} \cup \operatorname{int}\left(C_{1}\right)$ such that $\left.c_{2}\right|_{\{u, v\}}=c_{1}$.

Since $c_{2}$ is a $\left(Z_{3}, f\right)$-coloring and since $c_{2}\left(W_{1}\right) \neq b_{w_{1}}, c_{2}\left(w_{2}\right) \neq b_{w_{2}}$, we redefine $b_{w_{1}} \in Z_{3}-\left\{c_{2}\left(w_{1}\right), c_{2}\left(u^{\prime}\right)+f\left(w_{1} u^{\prime}\right)\right\}, b_{w_{2}} \in Z_{3}-\left\{c_{2}\left(w_{2}\right), c_{2}\left(v^{\prime}\right)+\right.$ $\left.f\left(w_{2} v^{\prime}\right)\right\}$.

By the minimality of $G$ again, $\left.c_{2}\right|_{\left\{u^{\prime}, v^{\prime}\right\}}$ can be extended to a $\left(Z_{3}, f\right)$ coloring $c_{3}$ of $C_{2} \cup \operatorname{int}\left(C_{2}\right)$ such that $\left.c_{3}\right|_{\left\{u^{\prime}, v^{\prime}\right\}}=c_{2}$ and $c_{3}\left(w_{1}\right) \neq b_{w_{1}}, c_{3}\left(w_{2}\right) \neq$ $b_{w_{2}}$. It follows that $c_{3}\left(w_{1}\right)=c_{2}\left(w_{1}\right)$ and $c_{3}\left(w_{2}\right)=c_{2}\left(w_{2}\right)$ and $c_{3}\left(x^{\prime}\right)-c_{3}\left(y^{\prime}\right) \neq$ $f\left(x^{\prime} y^{\prime}\right)$ for every directed edge $x^{\prime} y^{\prime} \in E\left(G_{2}\right)$. Combining $c_{2}$ and $c_{3}$, we obtained a required $\left(Z_{3}, f\right)$-coloring of $G$ extending $c_{1}$, contrary to (3).

Lemma 6.2.8 (i) There is no 3-path $x_{i+2} u^{\prime} v^{\prime} x_{j}$ for $j \in\{i+4, \ldots, m\}$ and there is no 3-path $x_{i-1} u^{\prime} v^{\prime} x_{j}$ for $j \in\{3, \ldots i-2\}$ where $u^{\prime}, v^{\prime} \in V(G)-V(C)$ and $x_{j} \in W$. 
(ii) There is no 2-path $x_{i+2} u^{\prime} x_{j}$ for $j \in\{i+4, \ldots, m\}$ and there is no 2-path $x_{i-1} u^{\prime} x_{j}$ for $j \in\{3, \ldots i-2\}$ where $u^{\prime} \in V(G)-V(C)$ and $x_{j} \notin W$.

Proof. Note that $x_{i+2} \notin W$. Assume that $P=x_{i+2} u^{\prime} v^{\prime} x_{j}$ is a 3-path satisfying the hypothesis of Lemma 6.2.8. Let $C_{1}$ be the cycle in $C \cup P$ containing $u^{\prime} v^{\prime}$ and $x y$ and let $C_{2}$ be the cycle in $C \cup P$ containing $u^{\prime} v^{\prime}$ and but not $x y$. Let $G_{i}=C_{i} \cup \operatorname{int}\left(C_{i}\right), i=1,2$. By the minimality of $G$, we can extended $c_{1}$ to a $\left(Z_{3}, f\right)$-coloring $c_{2}$ of $G_{1}$. Assume that $P$ is oriented from $x_{i+2}$ to $u^{\prime}$, from $u^{\prime}$ to $v^{\prime}$ and from $x_{j}$ to $v^{\prime}$.

Let $W^{\prime \prime}=\left(W \cap V\left(G_{2}\right)\right) \cup\left\{x_{i+2}\right\}$. Then $G_{2}$ satisfies the condition of Theorem 6.2.1. Define

$$
b_{z}^{\prime \prime}= \begin{cases}b_{z} & \text { if } z \in W^{\prime \prime}-\left\{x_{i+2}, x_{j}\right\} \\ b_{x_{i+2}} \in Z_{3}-\left\{c_{2}\left(x_{i+2}\right), c_{2}\left(u^{\prime}\right)+f\left(x_{i+2} u^{\prime}\right)\right\} & \text { if } z=x_{i+2} \\ b_{x_{j}} \in Z_{3}-\left\{c_{2}\left(x_{j}\right), c\left(v^{\prime}\right)+f\left(x_{j} v^{\prime}\right)\right\} & \text { if } z=x_{j} .\end{cases}
$$

By the minimality of $G,\left.c_{2}\right|_{\left\{u^{\prime}, v^{\prime}\right\}}$ can be extended to a $\left(Z_{3}, f\right)$-coloring $c_{3}$ of $G_{2}$ such that $c_{3}\left(w^{\prime \prime}\right) \neq b_{w^{\prime \prime}}$ for every $w^{\prime \prime} \in W^{\prime \prime}$. It follows that $c_{3}\left(x_{i+2}\right)=$ $c_{2}\left(x_{i+2}\right)$ and $c_{3}\left(x_{j}\right)=c_{2}\left(x_{j}\right)$. Combining $c_{2}$ and $c_{3}$, we get a required $\left(Z_{3}, f\right)$ coloring of $G$ extending $c_{1}$ such that $c(z) \neq b_{z}$ for each vertex $z \in W$, contrary to $(3)$.

The proofs for the case that there is no 3 -path $x_{i-1} u^{\prime} v^{\prime} x_{j}$ for $j \in\{3, \ldots, i-$ 2\} where $u^{\prime}, v^{\prime} \in V(G)-V(C)$ and $x_{j} \in W$ is similar.

Suppose that $Q=x_{i+2} u^{\prime} x_{j}$ is a 2-path satisfying the hypotheses of Lemma 6.2.8. If $x_{j}=x_{i+4}$, by (A1) the cycle $x_{i+4} x_{i+3} x_{i+4} u^{\prime} x_{i+2}$ is facial. Thus $d_{G}\left(x_{i+3}\right)=2$, contrary to Lemma 6.2.4.

Thus we assume that $j \in\{i+5, \ldots, m\}$. Let $C_{3}$ be the cycle in $C \cup Q$ containing $u^{\prime}$ and $x y$ and let $C_{4}$ be the cycle in $C \cup Q$ containing $u^{\prime}$ but not 
$x y$. Let $G_{i}=C_{i} \cup \operatorname{int}\left(C_{i}\right), i=3,4$. By the minimality of $G$, we can extend $c_{1}$ to a $\left(Z_{3}, f\right)$-coloring $c_{3}$ of $G_{3}$. Recall that $Q$ is oriented from $x_{i+2}$ to $u^{\prime}$ and from $x_{j}$ to $u^{\prime}$.

Let $W^{\prime \prime \prime}=\left(W \cap V\left(G_{4}\right)\right) \cup\left\{x_{i+2}\right\}$. By (W2) $x_{3} \notin W$. Thus $G_{4}\left[W^{\prime \prime \prime}\right]$ is edgeless. Let $b_{x_{i+2}} \in Z_{3}-\left\{c_{2}\left(x_{i+2}\right), c_{2}\left(u^{\prime}\right)+f\left(x_{i+2} u^{\prime}\right)\right\}$. Therefore both $G_{4}$ and $W^{\prime \prime}$ satisfy the hypotheses of Theorem 6.2.1. By the minimality of $G$ again, $\left.c_{2}\right|_{\left\{u^{\prime}, x_{j}\right\}}$ can be extended to a $\left(Z_{3}, f\right)$-coloring of $G_{4}$. Using $c_{3}$ and $c_{4}$, we can get a required $\left(Z_{3}, f\right)$-coloring of $G$, contrary to $(3)$.

The proofs for the case that there is no 2-path $x_{i-1} u^{\prime} x_{j}$ for $j \in\{3, \ldots, i-$ $2\}$ where $u^{\prime}, v^{\prime} \in V(G)-V(C)$ and $x_{j} \notin W$ is similar.

Lemma 6.2.9 G has no separating 5-cycle.

Proof. If $G$ has a separating 5-cycle $C^{\prime}$, then we apply the minimality of $G$ to extend $c_{1}$ to an group coloring of $G-\operatorname{int}\left(C^{\prime}\right)$. We then apply Corollary 6.2.3 to extend the coloring of $C^{\prime}$ to a group coloring of $C^{\prime} \cup \operatorname{int}\left(C^{\prime}\right)$. This contradiction proves Lemma 6.2.9.

If $u$ or $v$ (say $u$ ) is in $W$, we replace $W$ by $W-u$. So we can assume that $\{u, v\} \subset V(C)-W$.

Lemma 6.2.10 If $G[W]$ has the edge $x y$, then $i \geq 4$.

Proof. Suppose that $i=3$. Take $a_{y} \in Z_{3}-\left\{b_{y}, c_{1}(v)-f(v y)\right\}, a_{x} \in$ $Z_{3}-\left\{b_{x}, a_{y}-f(y x)\right\}$. Let $b_{z}=a_{x}-f(x z)$ for every $z \in N(x)-\{x, y\}$ and let $b_{z}=a_{y}-f(y z)$ for every $z \in N(y)-\{x, v\}$. Let $G^{\prime}=G-\{y, x\}$ and $W^{\prime}=W \cup N(x) \cup N(y)-\{x, y, v\}$. 
If $G[N(x) \cup N(y)]$ does not contain a 4-cycle, by (W2), $x_{6} \notin W$. By Lemma 6.2.6 $G\left[W^{\prime}\right]$ is edgeless and so both $G^{\prime}$ and $W^{\prime}$ satisfy the condition of Theorem 6.2.1. So assume that $G[N(x) \cup N(y)]$ contains a 4-cycle $x q_{1} q_{2} y x$, where $q_{1} \in N(x)$ and $q_{2} \in N(y)$ (possibly $q_{2}=v$ or $q_{1}=x_{i+2}$ ). By Lemma 6.2.6 and (A2), $G^{\prime}\left[W^{\prime}\right]$ contains at most one edge $q_{1} q_{2}$. If $G^{\prime}\left[W^{\prime}\right]$ has that edge $q_{1} q_{2}$, then $q_{2} \neq v$. If there is a 2-path $q_{2} q_{3} q_{4}$ where $q_{4} \in W^{\prime}$, then $q_{3} \neq q_{1}\left(q_{1}=x_{5}\right.$ possibly). By (A2) and (A3), $q_{4} \notin N\left(x_{3}\right) \cup N\left(x_{4}\right)$. Thus $q_{4} \in W$, contrary to Lemma 6.2.7. By (A2) $G\left[\left\{u, v, q_{1}, q_{2}\right\}\right]$ is not a 4-cycle. Therefore both $G^{\prime}$ and $W^{\prime}$ satisfy the condition of Theorem 6.2.1 with $q_{2}$ playing the role of $x$ of $G$.

By the minimality of $G, c_{1}$ can be extended to a $\left(Z_{3}, f\right)$-coloring $c_{2}$ of $G^{\prime}$ such that $\left.c_{2}\right|_{\{u, v\}}=c_{1}$ and $c_{2}\left(w^{\prime}\right) \neq b_{w^{\prime}}$ for each vertex $w^{\prime} \in W^{\prime}$.

Define $c: V(G) \mapsto Z_{3}$ by

$$
c(z)= \begin{cases}c_{2}(z) & \text { if } z \in V(G)-\{x, y\} \\ a_{x} & \text { if } z=x \\ a_{y} & \text { if } z=y\end{cases}
$$

Then $c$ is a required $\left(Z_{3}, f\right)$-coloring of $G$ such that $c(w) \neq b_{w}$ for each vertex $w \in W$ and $\left.c\right|_{\{u, v\}}=c_{1}$, contrary to $(3)$.

Lemma 6.2.11 If $x_{i-1} x_{i} x_{i+1} x_{i+2} x_{i-1}$ is a facial 4-cycle of $G$, then there is no 3-path $x_{i-1} q_{4} q_{3} x_{i+4}$, where $i \in\{4,5, \ldots, m-4\}$.

Proof. Assume that $P=x_{i-1} q_{4} q_{3} x_{i+4}$ exists. Let $C_{1}$ be the cycle in $C \cup$ $\left\{x_{i-1} q_{4}, q_{4} q_{3}, q_{3} x_{i+4}\right\}$ containing $x_{i-1} q_{4}, q_{4} q_{3}, q_{3} x_{i+4}$ and $u v$ and let $C_{2}$ be the cycle in $C \cup\left\{x_{i-1} q_{4}, q_{4} q_{3}, q_{3} x_{i+4}, x_{i-1} x_{i+2}\right\}$ containing $x_{i-1} q_{4}, q_{4} q_{3}, q_{3} x_{i+4}$ and $x_{i-1} x_{i+2}$ but not $u v$ nor $y x$. Let $G_{i}=C_{i} \cup \operatorname{int}\left(C_{i}\right), i=1,2$. 
By the minimality of $G, c_{1}$ can be extended to a $\left(Z_{3}, f\right)$-coloring $c_{2}$ of $G_{1}$. We claim that there is not a 2-path from $x_{i+4}$ to $x_{i-1}$ in $G_{2}$. By contradiction, assume that $x_{i+4} z x_{i-1}$ is a 2-path in $G_{2}$. Then we have two 5-cycles $x_{i-1} q_{4} q_{3} x_{i+4} z x_{i-1}$ and $x_{i-1} x_{i+2} x_{i+3} x_{i+4} z x_{i-1}$. By Lemma 6.2.9, $d_{G}(z)=2$, contrary to Lemma 6.2.4.

Define $a_{y} \in Z_{3}-\left\{b_{y}, c_{2}\left(x_{i-1}\right)-f\left(x_{i-1} y\right)\right\}, a_{x} \in Z_{3}-\left\{b_{x}, a_{y}-f\left(x_{i} x_{i+1}\right)\right\}, a_{x_{i+2}} \in$ $Z_{3}-\left\{c_{2}\left(x_{i-1}\right)-f\left(x_{i-1} x_{i-2}\right), a_{x}-f\left(x_{i+1} x_{i+2}\right)\right\}$. Let $W^{\prime \prime}=\left\{x_{i+4}, x_{i-1}, x_{i+2}\right\}, b_{x_{i+4}} \in$ $Z_{3}-\left\{c_{2}\left(x_{i+4}\right), c_{2}\left(q_{3}\right)+f\left(q_{3} x_{i+4}\right)\right\}, b_{x_{i-1}} \in Z_{3}-\left\{c_{2}\left(x_{i-1}\right), c_{2}\left(q_{4}\right)+f\left(x_{i-1} q_{4}\right), b_{x_{i+2}} \in\right.$ $Z_{3}-\left\{a_{x_{i+2}}, c_{2}\left(x_{i-1}\right)-f\left(x_{i-1} x_{i+2}\right)\right\}$. By (A2) $G\left[\left\{q_{3}, q_{4}, x_{i-1}, x_{i+2}\right\}\right]$ is not a 4-cycle. Therefore $G_{2}$ and $W^{\prime \prime}$ satisfy the hypotheses of Theorem 6.2.1 with $x_{i-1}$ playing the role of $x$ of $G$. By the minimality of $G,\left.c_{2}\right|_{\left\{q_{3}, q_{4}\right\}}$ can be extended to a $\left(Z_{3}, f\right)$-coloring $c_{3}$ of $G_{2}$ such that for every $w^{\prime \prime} \in W^{\prime \prime}$, $c_{3}\left(w^{\prime \prime}\right) \neq b_{w^{\prime \prime}}$.

Define $c: V(G) \mapsto Z_{3}$ by

$$
c(z)= \begin{cases}c_{2}(z) & \text { if } z \in V\left(G_{1}\right), \\ c_{3}(z) & \text { if } z \in V\left(G_{2}\right), \\ a_{x} & \text { if } z=x, \\ a_{y} & \text { if } z=y .\end{cases}
$$

Thus $c$ is a required $\left(Z_{3}, f\right)$-coloring of $G$, contrary to (3).

Lemma 6.2.12 If $G[W]$ has an edge, then $x_{i-2} \in W$ and hence $i \geq 5$.

Proof. Since $G[W]$ has only one edge, $x_{i-1} \notin W$. By contradiction, suppose that $x_{i-2} \notin W$.

Claim $1 G[N(x) \cup N(y)]$ does not contain a 4-cycle $x y x_{i-1} x_{i+2} x$. 
Proof. By contradiction, suppose that $G[N(x) \cup N(y)]$ contains a 4-cycle $x y x_{i-1} x_{i+2} x$. We consider two cases.

Case 1. $x_{i-3} \in W$.

Let $G^{\prime}=G-\left\{x_{i-2}, x_{i-1}, x_{i+2}, x, y\right\}$ and $W^{\prime}=W \cup N\left(x_{i-2}\right) \cup N\left(x_{i-1}\right) \cup$ $N\left(x_{i+2}\right)-\left\{x, y, x_{i-1}, x_{i-2}, x_{i+2}\right\}$. We assume that the edge $x_{i-1} x_{i+2}$ is oriented from $x_{i-1}$ to $x_{i+2}$. Let $a_{x_{i-2}}=b_{x_{i-3}}-f\left(x_{i-3} x_{i-2}\right), a_{x_{i-1}} \in Z_{3}-\left\{a_{x_{i-2}}-\right.$ $\left.f\left(x_{i-2} x_{i-1}\right)\right\}, a_{y} \in Z_{3}-\left\{b_{y}, a_{x_{i-1}}-f\left(x_{i-1} x_{i}\right)\right\}, a_{x} \in Z_{3}-\left\{b_{x}, a_{y}-f\left(x_{i} x_{i+1}\right)\right\}$ and $a_{x_{i+1}} \in Z_{3}-\left\{a_{x}-f\left(x_{i+1} x_{i+2}\right), a_{x_{i-1}}-f\left(x_{i-1} x_{i+1}\right)\right\}$. Define $b_{z}=a_{x_{j}}-$ $f\left(x_{j} z\right)$ if $z \in N\left(x_{j}\right), j \in\{i-1, i-2, i+2\}$. By (A2), $b_{z}$ is well defined. By (A2), (A3) and Lemma 6.2.6, $G^{\prime}\left[W^{\prime}\right]$ contains at most one edge $x_{i+3} x_{i+4}$. If $G^{\prime}\left[W^{\prime}\right]$ contains that edge, then $x_{i+4} \in W$. Assume that there is a 2-path $x_{i+4} q_{3} q_{4}$. By Lemma 6.2.5, $q_{4} \in W^{\prime}-W$. By (A3), $q_{4} \notin N\left(x_{i+2}\right)$. By Lemma 6.2.11, $q_{4} \notin N\left(x_{i-1}\right)$. So assume that $q_{4} \in N\left(x_{i-2}\right)$.

Let $Q=x_{i-2} q_{4} q_{3} x_{i+4}$ and $C_{3}$ be the cycle in $C \cup Q$ containing $u v$ and $C_{4}$ be the cycle in $C \cup Q$ not conaining uv. $G_{i}=C_{i} \cup \operatorname{int}\left(C_{i}\right), i=3,4$. By the minimality of $G, c_{1}$ can be extended to a $\left(Z_{3}, f\right)$-coloring $c_{2}$ of $G_{3}$.

Let $W_{2}=W \cap V\left(G_{4}\right) \cup\left\{x_{i-2}, x_{i+4}\right\}$. Assume that $Q$ is oriented from $x_{i-2}$ to $q_{4}$, from $q_{4}$ to $q_{3}$ and from $x_{i+4}$ to $q_{3}$. Since $c_{2}$ is a $\left(Z_{3}, f\right)$-coloring of $G_{3}$ and $b_{w} \neq c_{2}(w)$ for $w \in W \cap V\left(G_{3}\right), b_{x_{i+4}} \in Z_{3}-\left\{c_{2}\left(x_{i+4}\right), c_{2}\left(q_{3}\right)+f\left(q_{3} x_{i+2}\right)\right\}$. Let $b_{x_{i-2}} \in Z_{3}-\left\{c_{2}\left(x_{i-2}\right), c_{2}\left(q_{4}\right)+f\left(x_{i-2} q_{4}\right)\right\}$.

By Lemma 6.2.5, $G\left[\left\{q_{3}, q_{4}, x, y\right\}\right]$ is not a 4-cycle. Therefore both $G_{4}$ and $W_{2}$ satisfy the hypotheses of Theorem 6.2.1, $c_{2}:\left\{q_{3}, q_{4}\right\} \mapsto Z_{3}$ can be extended to a $\left(Z_{3}, f\right)$-coloring of $G_{4}$. Thus $c_{1}$ can be extended to a required $\left(Z_{3}, f\right)$-coloring of $G$, contrary to $(3)$. 
Case $2 x_{i-3} \notin W$.

Let $G^{\prime}=G-\{x, y\}$ and $W^{\prime}=W \cup\left\{x_{i-1}, x_{i+2}\right\}$. Take $a_{x} \in Z_{3}-\left\{b_{x}\right\}, a_{y} \in$ $Z_{3}-\left\{b_{y}, a_{x}+f(y x)\right\}, b_{x_{i-1}}=a_{y}+f\left(x_{i-1} y\right), b_{x_{i+2}}=a_{x}-f\left(x x_{i+2}\right)$. By (W2), $x_{i-1} x_{i+2}$ is the only edge of $G^{\prime}\left[W^{\prime}\right]$. By Lemma 6.2.6, there is no 2-path from $x_{i-1}$ to a vertex of $W$. By (A2), $G\left[\left\{u, v, x_{i-1}, x_{i+2}\right\}\right]$ is not a 4-cycle. Therefore both $G^{\prime}$ and $W^{\prime}$ satisfy the hypotheses of Theorem 6.2.1 with $x_{i-1}$ playing the role of $x$ of $G$. By the minimality of $G, c_{1}$ can be extended to a $\left(Z_{3}, f\right)$-coloring of $G^{\prime}$ and hence $c_{1}$ can be extended to a required $\left(Z_{3}, f\right)$ coloring of $G$, contrary to $(3)$.

Now we are ready to complete the proof of Lemma 6.2.12. Define $a_{y} \in$ $Z_{3}-\left\{b_{y}\right\}, a_{x} \in Z_{3}-\left\{b_{x}, a_{y}-f(y x)\right\}, G^{\prime}=G-\{x, y\}$ and $W^{\prime}=W \cup N(x) \cup$ $N(y)-\{x, y\}$. By (W2), $x_{i+3} \notin W^{\prime}$.

If $G[N(x) \cup N(y)]$ does not contain a 4-cycle, then by Lemma 6.2.6 and (W2), $G\left[W^{\prime}\right]$ is edgeless. So assume that $G[N(x) \cup N(y)]$ contains a 4-cycle $x q_{1} q_{2} y x$, where $q_{1} \in N(x)$ and $q_{2} \in N(y)$. By Lemma 6.2.6 and (A2), $G^{\prime}\left[W^{\prime}\right]$ contains only one edge $q_{1} q_{2}$ ( possibly $q_{1}=x_{i+2}$ or $q_{2}=x_{i-1}$ ). By Claim 2 , we assume that $q_{2} \neq x_{i-1}$. Suppose that there is a 2-path $q_{2} q_{3} q_{4}$, where $q_{4} \in W^{\prime}$. Since $G^{\prime}\left[W^{\prime}\right]$ has only one edge, $q_{3} \neq q_{1}$. By (A2) and (A3), $q_{4} \in$ $W-N(x) \cup N(y)$, contrary to Lemma 6.2.7. By (A2), $G^{\prime}\left[\left\{q_{1}, q_{2}, u, v\right\}\right]$ is not a 4-cycle. Therefore both $G^{\prime}$ and $W^{\prime}$ satisfy the hypotheses of Theorem 6.2.1 with $q_{2}$ playing the role of $x$ of $G$.

Let $b_{z}=a_{x}-f(x z)$ if $z \in N(x)-W$ and $b_{z}=a_{y}-f(y z)$ if $z \in N(y)-W$. By the minimality of $G, c_{1}$ can be extended to a $\left(Z_{3}, f\right)$-coloring $c_{2}$ of $G^{\prime}$ such 
that $c_{2}(w) \neq b_{w}, w \in W$. Define $c: V(G) \mapsto Z_{3}$ by

$$
c(z)= \begin{cases}c_{2}(z) & \text { if } z \in V(G)-\{x, y\}, \\ a_{x} & \text { if } z=x \\ a_{y} & \text { if } z=y .\end{cases}
$$

Then $c$ is a required $\left(Z_{3}, f\right)$-coloring satisfying $c(w) \neq b_{w}$ for each vertex $w \in W$ and extending $c_{1}$, contrary to (3). The proof for the case when $q_{1} \neq x_{i+2}$ is similar.

Lemma 6.2.13 $G[W]$ has no edge.

Proof. Suppose that $G[W]$ has the edge $x y$ where $x=x_{i+1}, y=x_{i}$. By Lemma 6.2 .12 and by (W2), $x_{i-1} \notin W$ and $x_{i-2} \in W$.

Case $1 G[N(x) \cup N(y)]$ contains no a 4-cycle.

Let $G^{\prime}=G-\left\{x_{i-1}, x_{i}, x_{i+1}\right\}$ and $W^{\prime}=W \cup N\left(x_{i-1}\right) \cup N\left(x_{i}\right) \cup N\left(x_{i+1}\right)-$ $\left\{x_{i-1}, x_{i}, x_{i+1}\right\}$. Let $a_{i-1}=b_{x_{i-2}}-f\left(x_{i-2} x_{i-1}\right), a_{i} \in Z_{3}-\left\{b_{x_{i}}, a_{i-1}-f\left(x_{i} x_{i-1}\right)\right\}$ and $a_{i+1} \in Z_{3}-\left\{b_{x_{i+1}}, a_{i}-f\left(x_{i} x_{i+1}\right)\right\}$. Let $b_{z}=a_{j}-f\left(x_{j} z\right)$ for every vertex $z \in\left(W^{\prime}-W\right) \cap\left(N\left(x_{i-1}\right) \cup N\left(x_{i}\right) \cup N\left(x_{i+1}\right)\right)$. Since $G[N(x) \cup N(y)]$ does not contain a 4-cycle, $b_{z}$ is well defined. Suppose that $G^{\prime}\left[W^{\prime}\right]$ has an edge $q_{1} q_{2}$ where $q_{1}, q_{2} \in W^{\prime}-W \subset N\left(x_{i-1}\right) \cup N\left(x_{i}\right) \cup N\left(x_{i+1}\right)$. We will distinguish the following two subcases.

Subcase $1.1 q_{2} \in N\left(x_{i-1}\right), q_{1} \in N\left(x_{i+1}\right)$.

Then $G$ has a 5 -cycle $q_{1} q_{2} x_{i-1} x_{i} x_{i+1} q_{1}$. Note that $q_{1}=x_{i+2}$ is possible. By Lemmas 6.2.4 and 6.2.9 $q_{2} \neq x_{i-2}$. By (A2), (A3), (W2) and Lemma 6.2.6, $q_{1} q_{2}$ is the only edge in $G^{\prime}\left[W^{\prime}\right]$. 
Assume that $G^{\prime}$ has a path $q_{1} q_{3} q_{4}$ where $q_{4} \in W^{\prime}$. As $G^{\prime}\left[W^{\prime}\right]$ has only one edge, $q_{3} \neq q_{2}$.

We claim that $q_{4} \in W$. By contradiction, suppose $q_{4} \in W^{\prime}-W$. If $q_{4} \in$ $N\left(x_{i-1}\right)-q_{2}$, then $G$ has two 5 -cycles $x_{i-1} x_{i} x_{i+1} q_{1} q_{2} x_{i-1}$ and $x_{i-1} q_{4} q_{3} q_{1} q_{2} x_{i-1}$. By Lemma 6.2.9, $d_{G}\left(q_{2}\right)=2$, contrary to Lemma 6.2.4. Since $G$ has a 5 -cycle $q_{1} q_{2} x_{i+1} x_{i} x_{i-1} q_{1}, q_{4} \notin N\left(x_{i}\right)$. By (A3), $q_{4} \notin N\left(x_{i+1}\right)$. Thus $q_{4} \in W$.

By Lemmas 6.2.5, 6.2.6 and 6.2.7, $q_{1}=x_{i+2}, q_{3}=x_{i+3}$ and $q_{4}=x_{i+4}$. It follows that $d_{G}\left(x_{i}\right)=d_{G}\left(x_{i+1}\right)=2$. Note that $q_{2} \neq x_{i-2}$.

Since $G^{\prime}$ is a subgraph of $G$, we have $G^{\prime} \in \mathcal{F}$ and $\left.f\right|_{V\left(G^{\prime}\right)} \in F\left(G, Z_{3}\right)$. We shall verify that both $G^{\prime}$ and $W^{\prime}$ satisfy the other hypotheses of Theorem 6.2.1 with $q_{1}$ and $q_{2}$ replacing $y$ and $x$, respectively.

We now assume that $G^{\prime}$ has a path $q_{2} q_{5} q_{6}$ where $q_{6} \in W^{\prime}$. By (A3), $q_{6} \notin W^{\prime}-W$ and hence $q_{6} \in W$. If $q_{5} \notin V(C)$, then it contradicts to Lemma 6.2.8(i). If $q_{5} \in V(C)$, then it contradicts to Lemma 6.2.8(ii). Thus there is no 2-path from $q_{2}$ to a vertex of $W^{\prime}$ in $G^{\prime}$.

By (A2) $G^{\prime}\left[\left\{u, v, q_{1}, q_{2}\right\}\right]$ is not a 4-cycle. Therefore both $G^{\prime}$ and $W^{\prime}$ satisfies the hypotheses of Theorem 6.2 .1 with $q_{2}$ playing the role of $x$ of $G$. Thus $c_{1}$ can be extended to a $\left(Z_{3}, f\right)$-coloring $c_{2}: V\left(G^{\prime}\right) \mapsto Z_{3}$ such that $\left.c_{2}\right|_{\{u, v\}}=c_{1}$ and $c_{2}\left(w^{\prime}\right) \neq b_{w^{\prime}}$ for every vertex $w^{\prime} \in W^{\prime}$.

Define $c: V(G) \mapsto Z_{3}$ by

$$
c(z)= \begin{cases}c_{2}(z) & \text { if } z \in V(G)-\left\{x_{i-1}, x_{i}, x_{i+1}\right\} \\ a_{j} & \text { if } z=x_{j}, j \in\{i-1, i, i+1\} .\end{cases}
$$

Then $c$ is a required $\left(Z_{3}, f\right)$-coloring satisfying $c(w) \neq b_{w}$ for every vertex $w \in W$ and extending $c_{1}$, contrary to (3).

Subcase $1.2 q_{2} \in N\left(x_{i-1}\right), q_{1} \in N\left(x_{i}\right)$. 
If $q_{2}=x_{i-2}$, then $d\left(x_{i-1}\right)=2$, contrary to Lemma 6.2.4. So $q_{2} \in V(G)-$ $V(C)$. Since $G[N(x) \cup N(y)]$ does not contain a 4-cycle, $q_{1} \neq x_{i+1}$. So we assume that $q_{2} \neq x_{i-2}$ and $q_{1} \neq x_{i+1}$. If there is a 2-path $q_{1} q_{3} q_{4}$, where $q_{4} \in W^{\prime}$, by (A3) and by Lemma 6.2.9 $q_{4} \notin N\left(x_{i+1}\right) \cup N\left(x_{i}\right) \cup N\left(x_{i-1}\right)$. Thus $q_{4} \in W$, contrary to Lemma 6.2.7. By (A2) $G^{\prime}\left[\left\{u, v, q_{1}, q_{2}\right\}\right]$ is not a 4-cycle.

Therefore both $G^{\prime}$ and $W^{\prime}$ satisfy the condition of Theorem 6.2.1 with $q_{1}$ playing the role of $x$ of $G$. Thus $c_{1}$ can be extended to a $\left(Z_{3}, f\right)$-coloring $c_{2}: V\left(G^{\prime}\right) \mapsto Z_{3}$ such that $\left.c_{2}\right|_{\{u, v\}}=c_{1}$ and $c_{2}\left(w^{\prime}\right) \neq b_{w^{\prime}}$ for every vertex $w^{\prime} \in W^{\prime}$.

Define $c: V(G) \mapsto Z_{3}$ by

$$
c(z)= \begin{cases}c_{2}(z) & \text { if } z \in V(G)-\left\{x_{i-1}, x_{i}, x_{i+1}\right\}, \\ a_{j} & \text { if } z=x_{j}, j \in\{i-1, i, i+1\} .\end{cases}
$$

Then $c$ is a required $\left(Z_{3}, f\right)$-coloring of $G$ such that $c(w) \neq b_{w}$ for every vertex $w \in W$, contrary to (3).

Case $2 G[N(x) \cup N(y)]$ contains a 4-cycle.

Without loss of generality, we assume that $x q_{1} q_{2} y x$ is a 4-cycle in $G[N(x) \cup$ $N(y)$ ], where $q_{1} \in N\left(x_{i+1}\right), q_{2} \in N\left(x_{i}\right)$. We distinguish the following two subcases.

Subcase $2.1 q_{2} \neq x_{i-1}$.

Let $G^{\prime}=G-\left\{x_{i-1}, x_{i}, x_{i+1}\right\}$ and $W^{\prime}=W \cup N\left(x_{i-1}\right) \cup N\left(x_{i}\right) \cup N\left(x_{i+1}\right)-$ $\left\{x_{i-1}, x_{i}, x_{i+1}\right\}$. Let $a_{i-1}=b_{x_{i-2}}-f\left(x_{i-2} x_{i-1}\right), a_{i} \in Z_{3}-\left\{b_{x_{i}}, a_{i-1}-f\left(x_{i} x_{i-1}\right)\right\}$ and $a_{i+1} \in Z_{3}-\left\{b_{x_{i+1}}, a_{i}-f\left(x_{i} x_{i+1}\right)\right\}$. Let $b_{z}=a_{j}-f\left(x_{j} z\right)$ for every vertex $z \in\left(W^{\prime}-W\right) \cap\left(N\left(x_{i-1}\right) \cup N\left(x_{i}\right) \cup N\left(x_{i+1}\right)\right)$. By (A2), $b_{z}$ is well defined.

Claim $2 G^{\prime}\left[W^{\prime}\right]$ has only one edge. 
Note that $q_{1} q_{2} \in G^{\prime}\left[W^{\prime}\right]$. Suppose that there is another edge $p_{1} p_{2}$ of $G^{\prime}\left[W^{\prime}\right]$. By (W2) $x_{i+3} \notin W$. It follows that $x_{i+2} x_{i+3} \notin E\left(G^{\prime}\left[W^{\prime}\right]\right)$. By Lemmas 6.2.5 and 6.2.6, we may assume that either $p_{2} \in N\left(x_{i-1}\right), p_{1} \in$ $N\left(x_{i+1}\right)$, or $p_{2} \in N\left(x_{i-1}\right), p_{1} \in N\left(x_{i}\right)$, or $p_{2} \in N\left(x_{i}\right), p_{1} \in N\left(x_{i+1}\right)$. Each case contradicts (A2) or (A3).

Claim 3 There is no 2-path from $q_{2}$ to every vertex of $W^{\prime}$.

By contradiction, suppose that $G^{\prime}$ has a path $q_{2} q_{3} q_{4}$ where $q_{4} \in W^{\prime}$. As $G^{\prime}\left[W^{\prime}\right]$ has only one edge, $q_{3} \neq q_{1}$. By (A2) and (A3), $q_{4} \notin N\left(x_{i-1}\right) \cup N\left(x_{i}\right) \cup$ $N\left(x_{i+1}\right)$. So $q_{4} \in W$, contrary to Lemma 6.2.7. So both $G^{\prime}$ and $W^{\prime}$ satisfy the condition of Theorem 6.2.1 with $q_{2}$ playing the role of $x$ of $G$. By the choice of $G, c_{1}$ can be extended to a $\left(Z_{3}, f\right)$ coloring $c_{2}$ of $G^{\prime}$. Define

$$
c(z)= \begin{cases}c_{2}(z) & \text { if } z \in V(G)-\left\{x_{i-1}, x_{i}, x_{i+1}\right\}, \\ a_{j} & \text { if } j \in\{i-1, i, i+1\} .\end{cases}
$$

Then $c$ is a required $\left(Z_{3}, f\right)$-coloring of $G$, contrary to (3).

Subcase $2.2 q_{2}=x_{i-1}$.

Let $G^{\prime}=G-\left\{x_{i-1}, y, x, q_{1}\right\}$ and $W^{\prime}=W \cup N\left(x_{i-1}\right) \cup N(x) \cup N\left(q_{1}\right)-$ $\left\{x_{i-1}, x, y, q_{1}\right\}$.

Note that we have defined that the edge $x_{j} z$ is oriented from $x_{j}$ to $z$ for every $z \in N\left(x_{j}\right)-V(C)$ if $q_{1} \neq x_{i+2}$. Assume that the edge $x_{i-1} q_{1}$ is oriented from $x_{i-1}$ to $q_{1}$ if $q_{1}=x_{i+2}$. Define $a_{x_{i-1}}=b_{x_{i-2}}-f\left(x_{i-2} x_{i-1}\right), a_{y} \in$ $Z_{3}-\left\{b_{y}, a_{x_{i-1}}-f\left(x_{i-1} y\right)\right\}, a_{x} \in Z_{3}-\left\{b_{x}, a_{y}-f(y x)\right\}, a_{q_{1}} \in Z_{3}-\left\{a_{x_{i-1}}-\right.$ $\left.f\left(x_{i-1} q_{1}\right), a_{x}-f\left(x q_{1}\right)\right\}$.

Assume that the edge $q_{1} z$ is oriented from $q_{1}$ to $z$, where $z \in N\left(q_{1}\right)-$ $\left(W \cup\left\{x_{i-1}\right)\right.$. Define $b_{z}=a_{\lambda}-f\left(a_{\lambda} z\right)$ where $\lambda \in\left\{x_{i-1}, q_{1}, x\right\}, z \in N\left(x_{i-1}\right) \cup$ 
$N\left(q_{1}\right) \cup N(x)-W$. By (A2), $b_{z}$ is well defined.

By (A2), (A3) and Lemma 6.2.9, $G^{\prime}\left[W^{\prime}\right]$ has at most one edge $x_{i+3} x_{i+4}$. If $G^{\prime}\left[W^{\prime}\right]$ contains that edge, then $x_{i+4} \in W, x_{i+2}=q_{1}$. If there is a 2-path $x_{i+4} q_{5} q_{6}$, where $q_{6} \in W^{\prime}$. By Lemma 6.2.7, $q_{6} \in W^{\prime}-W$. By (A3), $q_{6} \in$ $N\left(q_{2}\right)$, contrary to Lemma 6.2.11. By Lemma 6.2.4 $G^{\prime}\left[\left\{x_{i+3}, x_{i+4}, u, v\right\}\right]$ is not a 4-cycle. Therefore both $G^{\prime}$ and $W^{\prime}$ satisfy the hypotheses of Theorem 6.2.1 with $x_{i+4}$ playing the role of $x$ of $G$. By the minimality of $G, c_{1}$ can be extended to a $\left(Z_{3}, f\right)$-coloring of $G^{\prime}$ amd hence $c_{1}$ can be extended to a required $\left(Z_{3}, f\right)$-coloring of $G$, contrary to (3).

Lemma 6.2.14 $x_{4} \in W$.

Proof. Suppose that $x_{4} \notin W$. Let $G^{\prime}=G-x_{3}$ and $W^{\prime}=W \cup N\left(x_{3}\right)-$ $\left\{x_{2}, x_{3}\right\}$. Define

$$
a_{x_{3}}= \begin{cases}z \in Z_{3}-\left\{b_{x_{3}}, c_{1}\left(x_{2}\right)-f\left(x_{2} x_{3}\right)\right\} & \text { if } x_{3} \in W, \\ z \in Z_{3}-\left\{c_{1}\left(x_{2}\right)-f\left(x_{2} x_{3}\right)\right\} & \text { if } x_{3} \notin W .\end{cases}
$$

Let $b_{z}=c_{1}\left(x_{3}\right)-f\left(x_{3} z\right)$ if $z \in N\left(x_{3}\right)-x_{2}$. Then $b_{z}$ is well defined.

By Lemmas 6.2.5, 6.2.6 and 6.2.13, $G^{\prime}\left[W^{\prime}\right]$ has at most one edge, namely $x_{4} x_{5}$. If $G^{\prime}\left[W^{\prime}\right]$ contains $x_{4} x_{5}$, then $x_{5} \in W$. If there is a 2-path $x_{5} q_{5} q_{6}$, where $q_{6} \in W^{\prime}-W$, then $q_{6} \in N\left(x_{3}\right)-\left\{x_{4}\right\}, q_{5} \neq x_{4}$. $G$ has a 5-cycle $x_{3} x_{4} x_{5} q_{5} q_{6} x_{3}$. By Lemma 6.2.9, $d_{G}\left(x_{4}\right)=2$, contrary to Lemma 6.2.4. By (A1), $G\left[\left\{u, v, x_{4}, x_{5}\right\}\right]$ does not contain a 4-cycle.

So both $G^{\prime}$ and $W^{\prime}$ satisfy the hypotheses of Theorem 6.2.1 with $x_{5}$ playing the role of $x$ of $G$. By the minimality of $G, G^{\prime}$ has a $\left(Z_{3}, f\right)$-coloring and hence $c_{1}$ can be extended to a required $\left(Z_{3}, f\right)$-coloring of $G$, contrary to (3).

Lemma 6.2.15 $|C| \geq 6$. 
Proof. Suppose that $|C|=5$. Let $G^{\prime}=G-\left\{x_{3}, x_{4}\right\}$ and $W^{\prime}=W \cup N\left(x_{3}\right) \cup$ $N\left(x_{4}\right)-\left\{x_{2}, x_{3}, x_{4}\right\}$. Let $a_{x_{3}} \in Z_{3}-\left\{c_{1}\left(x_{2}\right)-f\left(x_{2} x_{3}\right)\right\}$ and $a_{x_{4}} \in Z_{3}-$ $\left\{b_{x_{4}}, a_{x_{3}}-f\left(x_{3} x_{4}\right)\right\}$. Put $b_{z}^{\prime}=a_{x_{i}}-f\left(x_{i} z\right)$ where $z \in N\left(x_{i}\right)-\left\{x_{2}, x_{3}, x_{4}\right\}, i=$ 3,4. By (A1), $b_{z}$ is well defined. Assume first that $G\left[N\left(x_{3}\right) \cup N\left(x_{4}\right)\right]$ does not contain a 4-cycle. It follows that $G^{\prime}\left[W^{\prime}\right]$ is edgeless. So assume that $G\left[N\left(x_{3}\right) \cup N\left(x_{4}\right)\right]$ contains a 4 -cycle and assume that $x_{3} q_{2} q_{1} x_{4} x_{3}$ is a 4 -cycle, where $q_{1} \in N\left(x_{4}\right), q_{2} \in N\left(x_{3}\right)$. By (A2) and Lemma 6.2.6, $G^{\prime}\left[W^{\prime}\right]$ has only one edge $q_{1} q_{2}$. If $q_{2}=x_{2}$, by (A1), $d_{G}\left(x_{3}\right)=2$, contrary to Lemma 6.2.4. So assume that $q_{2} \neq x_{2}$. Assume that $q_{1}=x_{5}$. If $G^{\prime}\left[W^{\prime}\right]$ has an edge $e$, by (A1) $e=x_{5} v^{\prime}$, where $v^{\prime} \in N\left(x_{3}\right)$. Thus $G$ has a 5 -cycle $x_{1} x_{2} x_{3} v^{\prime} x_{5} x_{1}$ and a 4 -cycle $x_{3} x_{4} x_{5} v^{\prime} x_{3}$. By Lemma 6.2.9 and (A1), $d_{G}\left(v^{\prime}\right)=2$, comtrary to Lemma 6.2.4.

Now assume that $q_{1} \neq x_{5}$ and $q_{2} \neq x_{2}$. By (A2) and (A3), there is no 2-path from $q_{2}$ to a vertex of $W^{\prime}$. So both $G^{\prime}$ and $W^{\prime}$ satisfy the hypotheses of Theorem 6.2.1 with $q_{2}$ playing the role of $x$ of $G$. By the minimality of $G$, $c_{1}$ can be extended to a $\left(Z_{3}, f\right)$-coloring of $G^{\prime}$, say $c_{2}$. Define

$$
c(z)= \begin{cases}c_{2}(z) & \text { if } z \in V(G)-\{x, y\}, \\ a_{x_{3}} & \text { if } z=x_{3} \\ a_{x_{4}} & \text { if } z=x_{4} .\end{cases}
$$

Then $c$ is a required $\left(Z_{3}, f\right)$-coloring of $G$, contrary to (3).

Lemma 6.2.16 $x_{6} \in W$.

Proof. Suppose that $x_{6} \notin W$. We will distinguish the following two cases.

Case $1 G\left[N\left(x_{3}\right) \cup N\left(x_{4}\right)\right]$ does not contain a 4-cycle. 
Let $a_{x_{3}} \in Z_{3}-\left\{c_{1}\left(x_{2}\right)-f\left(x_{2} x_{3}\right)\right\}, a_{x_{4}} \in Z_{3}-\left\{b_{x_{4}}, c_{1}\left(x_{3}\right)-f\left(x_{3} x_{4}\right)\right\}$. Put $G^{\prime}=G-\left\{x_{3}, x_{4}\right\}$ and $W^{\prime}=W \cup N\left(x_{3}\right) \cup N\left(x_{4}\right)-\left\{x_{2}, x_{3}, x_{4}\right\}$. Since $G\left[N\left(x_{3}\right) \cup N\left(x_{4}\right)\right]$ does not contain a 4-cycle, by Lemma 6.2.6 $G^{\prime}\left[W^{\prime}\right]$ is edgeless. Let $b_{z}=c_{1}\left(x_{j}\right)-f\left(x_{j} z\right)$ if $z \in\left(W^{\prime}-W\right) \cap\left(N\left(x_{3}\right) \cup N\left(x_{4}\right)\right)-\left\{x_{5}\right\}$. By (A1), $b_{z}$ is well defined.

By the choice of $G, c_{1}$ can be extended to a $\left(Z_{3}, f\right)$-coloring $c_{2}$ of $G^{\prime}$. Define

$$
c(z)= \begin{cases}c_{2}(z) & \text { if } z \in V(G)-\{x, y\} \\ a_{x_{3}} & \text { if } z=x_{3} \\ a_{x_{4}} & \text { if } z=x_{4} .\end{cases}
$$

Then $c$ is a required $\left(Z_{3}, f\right)$-coloring of $G$, violating to $(3)$.

Case $2 G\left[N\left(x_{3}\right) \cup N\left(x_{4}\right)\right]$ contains a 4-cycle.

Let $a_{x_{4}} \in Z_{3}-\left\{b_{x_{4}}\right\}, a_{x_{5}} \in Z_{3}-\left\{a_{x_{4}}-f\left(x_{4} x_{5}\right)\right\}$. Put $G^{\prime}=G-\left\{x_{4}, x_{5}\right\}$ and $W^{\prime}=W \cup N\left(x_{4}\right) \cup N\left(x_{5}\right)-\left\{x_{4}, x_{5}\right\}$. By (W2), $G\left[N\left(x_{4}\right) \cup N\left(x_{5}\right)\right]$ does not contains a 4-cycle.

If $x_{7} \notin W$, by (A2), $G^{\prime}\left[W^{\prime}\right]$ is edgeless. Assume that $x_{7} \in W$. By (A2) and Lemma $6.2 .5, G^{\prime}\left[W^{\prime}\right]$ has only one edge $x_{6} x_{7}$. If $G^{\prime}$ has a 2-path $x_{7} q_{5} q_{6}$, where $q_{6} \in W^{\prime}$, then by Lemma 6.2.6, $q_{6} \in N\left(x_{4}\right) \cup N\left(x_{5}\right)$. By (A3) and (A1), $q_{5} \neq x_{6}$. If $q_{6} \in N\left(x_{5}\right)$, then $G$ has a 5-cycle $x_{5} q_{6} q_{5} x_{7} x_{6} x_{5}$. By Lemma 6.2.9 $d_{G}\left(x_{6}\right)=2$, contrary to Lemma 6.2.4. By Lemma 6.2.6, $q_{6} \notin N\left(x_{4}\right)$. By Lemma 6.2.5, $G^{\prime}\left[\left\{u, v, x_{6}, x_{7}\right\}\right]$ is not a 4-cycle.

Therefore both $G^{\prime}$ and $W^{\prime}$ satisfy the condition of Theorem 6.2.1 with $x_{7}$ playing the role of $x$ of $G$. By the minimality of $G, c_{1}$ can be extended to a 
$\left(Z_{3}, f\right)$-coloring $c_{2}$ of $G^{\prime}$. Define

$$
c(z)= \begin{cases}c_{2}(z) & \text { if } z \in V(G)-\{x, y\}, \\ a_{x_{3}} & \text { if } z=x_{3}, \\ a_{x_{4}} & \text { if } z=x_{4} .\end{cases}
$$

Then $c$ is a required $\left(Z_{3}, f\right)$-coloring of $G$, violating to (3).

Proof of Theorem 6.2.1. Let $a_{x_{5}}=b_{x_{6}}+f\left(x_{5} x_{6}\right), a_{x_{4}} \in Z_{3}-\left\{b_{x_{4}}, a_{x_{5}}+\right.$ $\left.f\left(x_{4} x_{5}\right)\right\}$. Put $G^{\prime}=G-\left\{x_{4}, x_{5}\right\}$ and $W^{\prime}=W \cup N\left(x_{4}\right) \cup N\left(x_{5}\right)-\left\{x_{4}, x_{5}\right\}$. Let $b_{z}=a_{x_{j}}-f\left(x_{j} z\right)$ if $z \in N\left(x_{j}\right), 3 \leq j \leq 5$. By (A1) $b_{z}$ is well defined.

If $G\left[N\left(x_{4}\right) \cup N\left(x_{5}\right)\right]$ does not contain a 4-cycle, by Lemma 6.2.6 $G^{\prime}\left[W^{\prime}\right]$ is edgeless. Assume that $G\left[N\left(x_{4}\right) \cup N\left(x_{5}\right)\right]$ contains a 4-cycle. By (A2) and Lemma 6.2.5, $G^{\prime}\left[W^{\prime}\right]$ contains only edge $q_{1} q_{2}$, where $q_{2} \in N\left(x_{4}\right)$ and $q_{1} \in N\left(x_{5}\right)$. By Lemma 6.2.4, $q_{1} \neq x_{6}$. Let $q_{2} q_{3} q_{4}$ be a 2-path where $q_{4} \in W^{\prime}$. By (A2) and (A3), $q_{4} \in W$, contrary to Lemma 6.2.7. Therefore both $G^{\prime}$ and $W^{\prime}$ satisfy the condition of Theorem 6.2.1 with $q_{2}$ playing the role of $x$ of $G$.

By the minimality of $G, c_{1}$ can be extended to a $\left(Z_{3}, f\right)$-coloring of $G^{\prime}$, say $c_{2}$. Define

$$
c(z)= \begin{cases}c_{2}(z), & \text { if } z \in V(G)-\{x, y\} \\ a_{x_{3}} & \text { if } z=x_{3} \\ a_{x_{4}} & \text { if } z=x_{4}\end{cases}
$$

Then $c$ is a required $\left(Z_{3}, f\right)$-coloring of $G$, contrary to (3).

The proof of Theorem 6.2.1 is complete.

Let $H, G$ be graphs and denote $H \subseteq G$ if $H$ is a subgraph of $G$. Let $A$ be a group. Given an $f \in F(G, A)$, if for an $\left(A,\left.f\right|_{E(H)}\right)$-coloring $c_{0}$ of $H$, there 
is an $(A, f)$-coloring $c$ of $G$ such that $c$ is an extension of $c_{0}$, then we say that $c_{0}$ is extended to $c$. If any $\left(A,\left.f\right|_{E(H)}\right)$-coloring $c_{0}$ of $H$ can be extended to an $(A, f)$-coloring $c$, then we say that $(G, H)$ is $(A, f)$-extensible. If for any $f \in F(G, A),(G, H)$ is $(A, f)$-extensible, then $(G, H)$ is $A$-extensible.

Corollary 6.2.17 Let $G \in \mathcal{F}$ be a simple planar graph and let $H=K_{2}$. Then $(G, H)$ is $Z_{3}$-extensible.

Proof. Let $f \in F\left(G, Z_{3}\right)$ and $V(H)=\left\{v_{1}, v_{2}\right\}$ and let $c_{0}: V(H) \mapsto Z_{3}$ is a $\left(Z_{3}, f\right)$-coloring of $H$. We may assume that in an plane embedding of $G$, the only edge in $E(H)$ is on the exterior cycle of $G$. By Theorem 6.2.1, $c_{0}$ can be extended to a $\left(Z_{3}, f\right)$-coloring of $G$.

\section{3 $Z_{3}$-coloring of $K_{3,3}$-minor free graphs}

Let $G_{1}$ and $G_{2}$ be two bridgeless graphs and $u_{1}, v_{1} \in V\left(G_{1}\right), u_{2}, v_{2} \in V\left(G_{2}\right)$. We define $G$ as follows: identify $u_{1}$ with $u_{2}$ and $v_{1}$ with $v_{2}$. We define these two new vertices $u$ and $v$. Then $G$ is called 2-sum of $G_{1}$ and $G_{2}$. Similarly let $u \in V\left(G_{1}\right)$ and $v \in V\left(G_{2}\right)$. $G$ is obtained from $G_{1}$ and $G_{2}$ by identifying $u$ with $v$ and $G$ is called 1-sum of $G_{1}$ and $G_{2}$.

Theorem 6.3.1 (Hall [28]) Let $G$ be a graph without $K_{3,3}$ minor. One of the followings must holds.

(1) $G$ is a planar graph;

(2) $G \cong K_{5}$ and

(3) for some $i \in\{1,2\}, G$ is the $i$-sum of two $G_{1}$ and $G_{2}$ such that $G_{1}$ and $G_{2}$ are proper minor of $G$. 
Lemma 6.3.2 Let $G$ be a graph with a set cut $S$ such that $G=G_{1} \cup G_{2}$ and $G_{1} \cap G_{2}=G[S]$. If $S=\{u\}$ and both $G_{1}$ and $G_{2}$ are $\left(Z_{3}, f\right)$-coloring, then $G$ is a $\left(Z_{3}, f\right)$-coloring.

Proof. Let $f \in F\left(G, Z_{3}\right)$. Then there is $c_{1}: V\left(G_{1}\right) \mapsto Z_{3}$ such that for every directed edge $x y \in E\left(G_{1}\right), c_{1}(x)-c_{1}(y) \neq f(x y)$ and there is $c_{2}: V\left(G_{2}\right) \mapsto Z_{3}$ such that for every directed edge $z w \in E\left(G_{2}\right), c_{2}(z)-c_{2}(w) \neq f(z w)$. It follows that there is $a \in Z_{3}$ such that $c_{1}(u)=c_{2}(u)+a$. Define

$$
c(z)=\left\{\begin{array}{l}
c_{1}(z), \text { if } z \in V\left(G_{1}\right) \\
c_{2}(z)+a \text { if } z \in V\left(G_{2}\right) .
\end{array}\right.
$$

Then $c$ is a $\left(Z_{3}, f\right)$-coloring of $G$.

Theorem 6.3.3 Suppose that $G$ is a connected $K_{3,3}$-minor free graph with girth $\geq 5$. Then there is a subgraph $H=K_{2}$ of $G$ such that $(G, H)$ is $Z_{3}$-extensible.

Proof. By contradiction, suppose that $G$ is an counterexample with $|V(G)|$ minimized.

By Theorems 6.2.1 and 6.3.1 and Lemma 6.3.2, we may assume that $G$ is 2-connected and $G$ can be represented as a 2 -sum of $G_{1}$ and $G_{2}$ such that $G_{1}$ and $G_{2}$ are proper minor of $G$ and assume that $G_{1}$ is planar with $\left|V\left(G_{1}\right)\right|$ minimized. Since $G$ has girth $\geq 5$, we may assume that $\left|V\left(G_{1}\right)\right| \geq 5$.

Since $G_{2}$ is a proper subgraph of $G$, pick $e=u^{\prime} v^{\prime} \in E\left(G_{2}\right)-E\left(G_{1}\right)$ and denote $H=G\left[\left\{u^{\prime}, v^{\prime}\right\}\right]$. Let $c_{0}:\{u, v\} \mapsto Z_{3}$ such that $c_{0}$ is a $\left(Z_{3}, f\right)$-coloring of $H$.

Let $V\left(G_{1}\right) \cap V\left(G_{2}\right)=\{u, v\}$. If $u v \in E(G)$, by the minimality of $G, c_{0}$ can be extended to a $\left(Z_{3}, f\right)$-coloring $c_{1}$ of $G_{2}$. By Corollary 6.2.17, $\left.c_{1}\right|_{\{u, c\}}$ 
can be extended to a $\left(Z_{3}, f\right)$-coloring of $G_{1}$. So $c_{0}$ can be extended to a $\left(Z_{3}, f\right)$-coloring of $G$, a contradiction.

So we assume that $u v \notin E(G)$. We claim that $G_{1}+u v$ is also planar. By contradiction, we assume that $G_{1}+u v$ is not planar. If $G_{1}+u v$ has a $K_{3,3}$-minor $\Gamma$, then new edge $u v \in E(\Gamma)$ since $G_{1}$ does not have a $K_{3,3^{-}}$ minor. Since $G$ is 2 -connected and since $\{u, v\}$ is 2-vertex cut of $G, G_{2}$ must have a $(u, v)$-path. It then follows that $G$ has a $K_{3,3}$-minor, contrary to the assumption of $G$. Therefore $G_{1}+u v$ does not have a $K_{3,3}$-minor. By Theorem 6.3.1, $G_{1}+u v \cong K_{5}$. contrary to the assumption that $G$ has girth at least 5. Thus $G_{1}+u v$ must be planar. Define $G^{*}$ obtained from $G$ by adding two new vertices $v_{1}, v_{2}$ and three new edges $u v_{1}, v_{1} v_{2}, v_{2} v$ such that $G_{i}^{*}$ is obtained from $G_{i}$ by adding these two vertices and these three edges ( $1 \leq i \leq 2)$. It follows that $G_{1}^{*}$ is planar and $G^{*}, G_{1}^{*}$ and $G_{2}^{*}$ have girth $\geq 5$. Assume that the path $u v_{2} v_{1} v$ is oriented from $u$ to $v_{1}$, from $v_{1}$ to $v_{2}$, from $v_{2}$ to $v$. Define $f_{1}: E\left(G^{*}\right) \mapsto Z_{3}$ by

$$
f_{1}(e)= \begin{cases}f(e), & \text { if } e \in E(G) \\ 0 & \text { if } e \in\left\{u v_{1}, v_{1} v_{2}, v_{2} v\right\} .\end{cases}
$$

Note that $u^{\prime} v^{\prime} \in E\left(G_{2}^{*}\right)-E\left(G_{1}^{*}\right)$ and $\left|V\left(G_{2}^{*}\right)\right|=|V(G)|-\left|V\left(G_{1}\right)\right|+2+2<$ $|V(G)|$. By the minimality of $G, c_{0}$ can be extended to a $\left(Z_{3}, f\right)$-coloring $c_{1}$ of $G_{2}^{*}$.

Now we reimbed $G_{1}^{*}$ in the plane such that the edges $u v_{1}, v_{1} v_{2}, v_{2} v$ are in the exterior cycle of $G_{1}^{*}$. Let $W=\{u, v\}$ and define $b_{u} \in Z_{3}-\left\{c_{1}(u), c_{1}\left(v_{1}\right)+\right.$ $\left.f_{1}\left(u v_{1}\right)\right\}, b_{v} \in Z_{3}-\left\{c_{1}(v), c_{1}\left(v_{2}\right)-f_{1}\left(v_{2} v\right)\right\}$. By Theorem 6.2.1, $\left.c_{1}\right|_{\left\{v_{1}, v_{2}\right\}} \longmapsto Z_{3}$ can be extended to a $\left(Z_{3}, f_{1}\right)$-coloring $c_{2}$ of $G_{1}^{*}$ such that $\left.c_{2}\right|_{\{u, v\}}=\left.c_{1}\right|_{\{u, v\}}$ and $c_{2}(u) \neq b_{u}, c_{2}(v) \neq b_{v}$. It follows that $c_{2}(u)=c_{1}(u)$ and $c_{2}(v)=c_{1}(v)$. 
Define

$$
c(z)=\left\{\begin{array}{l}
c_{1}(z) \text { if } z \in V\left(G_{1}\right) \\
c_{2}(z) \text { if } z \in V\left(G_{2}\right) .
\end{array}\right.
$$

Then $c$ is a $\left(Z_{3}, f\right)$-coloring of $G$, a contradiction. 


\section{Chapter 7}

\section{3-list coloring of planar graphs with girth at least 4}

\subsection{Introduction}

Let $G$ be a graph and let $L(v)$ be a set of allowed colors for each vertex $v$. An $L$-list coloring of a graph $G$ is a proper vertex coloring in which every vertex $v$ gets a color from $L(v)$. $G$ is $k$-choosable if $G$ has a list coloring for each list assignment with $k$ colors in each list.

The concept of $L$-list coloring, choosability and choice number was introduced by Erdos, Rubin and Taylor [22] in 1979 and Vizing [53] in 1976.

Alon and Tarsi [2] proved that every bipartite planar graph is 3-choosable. Thomassen [48] showed that every planar graph is 5-choosable. Voigt [54] presented an example of a planar graph which is not 4-choosable.

Thomassen [49] proved the following theorem. 
Theorem 7.1.1 Every planar graph of girth at least 5 is 3-choosable.

In this chapter, we use a similar technique to prove the following result which extends Thomassen's result [49].

Theorem 7.1.2 Suppose that $G$ is a planar graph with girth at least 4 such that all 4-cycles are independent and every 4-cycle is facial. If the minimum distance between 4-cycles and 5-cycles is at least 1, then $G$ is 3-choosable.

The conditions of Theorem 7.1.2 cannot be relax. The Gutner [26]'s example with only 164 vertices is not 3 -choosable but there exist a 5 -cycle adjacent a 4-cycle.

Theorem 7.1.3 If $G$ is a connected $K_{3,3}$-minor free graph with girth at least 5, then $G$ is 3-choosable.

Let $G$ and $H$ be two graphs. $G$ is contractible to $H$ if $H$ can be obtained from $G$ by contracting some edges of $G$ and deleting the resulting loops. If $G$ contains a subgraph which is contractible to $H$, then $H$ is a minor of $G$. A set of graphs is said to be independent if no two of them have common vertex.

\subsection{A list coloring Theorem}

Let $\mathcal{F}$ denote the set of connected graphs such that a graph $G \in \mathcal{F}$ if and only if each of the following holds.

(A1) $G$ is a planar with girth $\geq 4$ and every 4 -cycle is facial;

(A2) all 4-cycles are independent.

(A3) the minimum distance between 4-cycles and 5-cycles is at least 1. 
Theorem 7.2.1 Let $G$ be a planar graph of girth at least 5. Let $A$ be a set of vertices in $G$ such that each vertex of $A$ is on the outer face boundary.

Assume that either

(B1) $G[A]$ has no edge or

(B2) $G[A]$ has precisely one edge $x y$ and $G$ has no 2-path from $x$ to a vertex of $A$.

Assume that $u, v$ be any adjacent vertices in $G$ both on the outer face boundary, that $G[\{u, v, x, y\}]$ is not a 4-cycle if $x y$ is an edge of $G[A]$ and that $L$ is color assignment such that $|L(w)| \geq 2$ for each vertex $w \in V(G)$ and $|L(w)| \geq 3$ for each vertex $w \in V(G)-A$. Let $c(u)$, $c(v)$ be distinct colors in $l(u)$ and $L(v)$ respectively. Then $c$ can be extended to a list coloring of $G$.

Corollary 7.2.2 Let $G \in \mathcal{F}$ with outer cycle $C: x_{1} x_{2} x_{3} x_{4} x_{5} x_{1}$. Let L be a color assignment such that $|L(v)| \geq 3$ for each vertex $v$ of $G$ and let $c$ be any list coloring of $C$. Then $c$ can be extended to a list coloring of $G$.

In order to prove Theorem 7.2.1, we first prove some lemmas. The following lemmas have the same hypotheses of Theorem 7.2.1 with an additional assumption that

$G$ is a counterexample to Theorem 7.2.1 and $|V(G)|$ is minimized.

Since $c$ can be easily extended to a list-coloring for every forest and a 4-cycle which satisfy the condition of Theorem 7.2.1, we may assume that $|V(G)| \geq 5$.

Lemma 7.2.3 $\kappa(G) \geq 2$. If $z \in V(G)-A$ and $z \notin\{u, v\}$, then $d_{G}(z) \geq 3$. 
Proof. If $G$ is not 2-connected, then $G$ has a block $B$ containing the edge $u v$. By the minimality of $G, c$ can be extended to a list coloring of $B$. Let $B_{1}$ be the block which has a common vertex $w_{1}$ with $B$ and pick its adjacent vertex $w_{2}$ in the outer cycle of $B_{1}$. Assume that put $c\left(w_{2}\right) \in L\left(w_{2}\right)$ such that $c\left(w_{1}\right) \neq c\left(w_{2}\right)$. Then $c$ can be extended to a list coloring of $B_{1}$, and so on. This contradicts to (1).

By contradiction, suppose that there is a $z_{0} \in V(G)-A$ such that $z_{0} \notin$ $\{u, v\}$ and $d_{G}\left(z_{0}\right)=2$. Let $G_{1}=G-z_{0}$. Denote $N\left(z_{0}\right)=\left\{z_{1}, z_{2}\right\}$. By the minimality of $G, c$ can be extended to a list coloring of $G_{1}$. Pick $c\left(z_{0}\right) \in$ $L\left(z_{0}\right)-\left\{c\left(z_{1}\right), c\left(z_{2}\right)\right\}$. Thus we have a list coloring of $G$, violating to (1).

By Lemma 7.2.3, we assume that $G$ is 2 -connected and $C: x_{1} x_{2} \ldots x_{m} x_{1}$ is the outer cycle of $G$. Let $u=x_{1}$ and $v=x_{2}$. If $G[W]$ has an edge $x y$, we can assume that $y=x_{i}, x=x_{i+1}$, where $3 \leq i \leq m-1$.

Lemma 7.2.4 $C$ has no chord $u^{\prime} v^{\prime}$ for which $G\left[\left\{u^{\prime}, v^{\prime}, x, y\right\}\right]$ is not a 4 -cycle.

Proof. Let $C_{1}$ be the cycle in $C \cup\left\{u^{\prime} v^{\prime}\right\}$ containing $u^{\prime} v^{\prime}$ and $u v$. Let $C_{2}$ be the cycle in $C \cup\left\{u^{\prime} v^{\prime}\right\}$ containing $u^{\prime} v^{\prime}$ but not $u v$. Then both $C_{1} \cup \operatorname{int}\left(C_{1}\right) \in \mathcal{F}$ and $C_{2} \cup \operatorname{int}\left(C_{2}\right) \in \mathcal{F}$. By the minimality of $G$ we can extend $c$ to a list coloring of $C_{1} \cup \operatorname{int}\left(C_{1}\right)$. Then the coloring of $u^{\prime}$ and $v^{\prime}$ can be extended to a list coloring of $C_{2} \cup \operatorname{int}\left(C_{2}\right)$, violating to (1).

Lemma 7.2.5 For $v^{\prime} \in V(G)-V(C), u^{\prime} \in V(C)$ and $w \in A, G$ has no path $u^{\prime} v^{\prime} w$ which satisfies one of the following

(1) $w \notin\{x, y\}$ or

(2) if $w \in\{x, y\}, G\left[\left\{u^{\prime}, v^{\prime}, x, y\right\}\right]$ is not a 4 -cycle. 
Proof. If $u^{\prime} v^{\prime} w$ exists, then we define $C_{1}$ and $C_{2}$ as follows: Let $C_{1}$ be the cycle in $C \cup\left\{u^{\prime} v^{\prime}, v^{\prime} w\right\}$ containing $u^{\prime} v^{\prime}, v^{\prime} w$ and $u v$ and let $C_{2}$ be the cycle in $C \cup\left\{u^{\prime} v^{\prime}, v^{\prime} w\right\}$ containing $u^{\prime} v^{\prime}, v^{\prime} w$ but not $u v$. By the minimality of $G$, $c$ can be extended to a list coloring of $C_{1} \cup \operatorname{int}\left(C_{1}\right)$. We replace $L(w)$ by $\left\{c\left(v^{\prime}\right), c(w)\right\}$ and then extend the coloring of $u^{\prime}$ and $v^{\prime}$ to a 3-list coloring of $C_{2} \cup \operatorname{int}\left(C_{2}\right)$. It follows that $w$ gets the color $c(w)$. Therefore we obtain a list coloring of $G$, contrary to (1).

Lemma 7.2.6 $G$ does not have 3-path $w_{1} u^{\prime} v^{\prime} w_{2}$ with $w_{1}, w_{2} \in A$ and $u^{\prime}, v^{\prime} \in$ $V(G)-A$ unless $\left\{w_{1}, w_{2}\right\}=\{x, y\}$ and $G\left[\left\{w_{1}, w_{2}, u^{\prime}, v^{\prime}\right\}\right]$ is a 4 -cycle.

Proof. By Lemma 7.2.5, assume that $u^{\prime}, v^{\prime} \in V(G)-V(C)$ and that $G$ has such a 3-path $w_{1} u^{\prime} v^{\prime} w_{2}$ with $w_{1}, w_{2} \in A$. Assume that the path $w_{1} u^{\prime} v^{\prime} w_{2}$ is oriented by from $w_{1}$ to $u^{\prime}$, from $u^{\prime}$ to $v^{\prime}$ and from $w_{2}$ to $v^{\prime}$. Define $C_{1}$ and $C_{2}$ as follows: Let $C_{1}$ be the cycle in $C \cup\left\{w_{1} u^{\prime}, u^{\prime} v^{\prime}, v^{\prime} w_{2}\right\}$ containing $w_{1} u^{\prime}, u^{\prime} v^{\prime}, v^{\prime} w_{2}$ and $u v$ and let $C_{2}$ be the cycle in $C \cup\left\{w_{1} u^{\prime}, u^{\prime} v^{\prime}, v^{\prime} w_{2}\right\}$ containing $w_{1} u^{\prime}, u^{\prime} v^{\prime}$ and $v^{\prime} w_{2}$ but not $u v$.

By the minimality of $G, c$ can be extended to a list coloring of $C_{1} \cup \operatorname{int}\left(C_{1}\right)$. We replace $L\left(w_{1}\right)$ by $\left\{c\left(w_{1}\right), c\left(u^{\prime}\right)\right\}$ and $L\left(w_{2}\right)$ by $\left\{c\left(w_{2}\right), c\left(v^{\prime}\right)\right\}$. By the minimality of $G$ again, the colors of $u^{\prime}$ and $v^{\prime}$ can be extended to a list coloring of $C_{2} \cup \operatorname{int}\left(C_{2}\right)$. It follows that $w_{1}$ gets the color $c\left(w_{1}\right)$ and $w_{2}$ gets the color $c\left(w_{2}\right)$. Therefore we obtained a list coloring of $G$, contrary to (1).

Lemma 7.2.7 (i) There is no 3-path $x_{i+2} u^{\prime} v^{\prime} x_{j}$ for $j \in\{i+4, \ldots, m\}$ and there is no 3-path $x_{i-1} u^{\prime} v^{\prime} x_{j}$ for $j \in\{3, \ldots i-2\}$ where $u^{\prime}, v^{\prime} \in V(G)-V(C)$ and $x_{j} \in A$. 
(ii) There is no 2-path $x_{i+2} u^{\prime} x_{j}$ for $j \in\{i+4, \ldots, m\}$ and there is no 2-path $x_{i-1} u^{\prime} x_{j}$ for $j \in\{3, \ldots i-2\}$ where $u^{\prime} \in V(G)-V(C)$ and $x_{j} \notin A$.

Proof. By (B2), $x_{i+2} \notin A$. Assume that $P=x_{i+2} u^{\prime} v^{\prime} x_{j}$ is a 3 -path satisfying the hypothesis of Lemma 7.2.7. Let $C_{1}$ be the cycle in $C \cup P$ containing $u^{\prime} v^{\prime}$ and $x y$ and let $C_{2}$ be the cycle in $C \cup P$ containing $u^{\prime} v^{\prime}$ and but not $x y$. Let $G_{i}=C_{i} \cup \operatorname{int}\left(C_{i}\right), i=1,2$. By the minimality of $G$, we can extend $c$ to a list coloring of $G_{1}$.

Let $A^{\prime \prime}=\left(A \cap V\left(G_{2}\right)\right) \cup\left\{x_{i+2}\right\}$. Then $G_{2}$ satisfies the condition of Theorem 7.2.1. We replace $L\left(x_{i+2}\right)$ by $\left\{c\left(x_{i+2}\right), c\left(u^{\prime}\right)\right\}$ and $L\left(x_{j}\right)$ by $\left\{c\left(x_{j}\right), c\left(v^{\prime}\right)\right\}$. By the minimality of $G$, the colors of $u^{\prime}$ and $v^{\prime}$ can be extended to a list coloring of $G_{2}$. It follows that $x_{i+2}$ gets the color $c\left(x_{i+2}\right)$ and $x_{j}$ gets the color $c\left(x_{j}\right)$. Therefore we get a list coloring of $G$, contrary to (1).

The proofs for the case that there is no 3 -path $x_{i-1} u^{\prime} v^{\prime} x_{j}$ for $j \in\{3, \ldots, i-$ $2\}$ where $u^{\prime}, v^{\prime} \in V(G)-V(C)$ and $x_{j} \in A$ is similar.

Suppose that $Q=x_{i+2} u^{\prime} x_{j}$ is a 2-path satisfying the hypotheses of Lemma 7.2.7. If $x_{j}=x_{i+4}$, by (A1) the cycle $x_{i+4} x_{i+3} x_{i+4} u^{\prime} x_{i+2}$ is facial. Thus $d_{G}\left(x_{i+3}\right)=2$, contrary to Lemma 7.2.3.

Thus we assume that $j \in\{i+5, \ldots, m\}$. Let $C_{3}$ be the cycle in $C \cup Q$ containing $u^{\prime}$ and $x y$ and let $C_{4}$ be the cycle in $C \cup Q$ containing $u^{\prime}$ but not $x y$. Let $G_{i}=C_{i} \cup \operatorname{int}\left(C_{i}\right), i=3,4$. By the minimality of $G$, we can extend $c$ to a list coloring of $G_{3}$.

Let $A^{\prime \prime \prime}=\left(A \cap V\left(G_{4}\right)\right) \cup\left\{x_{i+2}\right\}$. By (B2), $x_{3} \notin W$. Thus $G_{4}\left[A^{\prime \prime \prime}\right]$ is edgeless. Repalce $L\left(x_{i+2}\right)$ by $\left\{c\left(x_{i+2}\right), c\left(u^{\prime}\right)\right\}$. Therefore both $G_{4}$ and $W^{\prime \prime}$ satisfy the hypotheses of Theorem 7.2.1. By the minimality of $G$ again, the colors of $u^{\prime}$ and $v^{\prime}$ can be extended to a list coloring of $G_{4}$. Therefore we can 
get a list coloring of $G$, contrary to (1).

The proofs for the case that there is no 2-path $x_{i-1} u^{\prime} x_{j}$ for $j \in\{3, \ldots, i-$ $2\}$ where $u^{\prime}, v^{\prime} \in V(G)-V(C)$ and $x_{j} \notin A$ is similar.

Lemma 7.2.8 G has no separating 5-cycle.

Proof. If $G$ has a separating 5 -cycle $C^{\prime}$, then we apply the minimality of $G$ to extend $c_{1}$ to a list coloring of $G-\operatorname{int}\left(C^{\prime}\right)$. We then apply Corollary 7.2.2 to extend the coloring of $C^{\prime}$ to a group coloring of $C^{\prime} \cup \operatorname{int}\left(C^{\prime}\right)$. This contradiction proves Lemma 7.2.8.

If $u$ or $v$ (say $u$ ) is in $A$, we replace $A$ by $A-u$. So we can assume that $\{u, v\} \subset V(C)-A$.

Lemma 7.2.9 If $G[A]$ has the edge $x y$, then $i \geq 4$.

Proof. Suppose that $i=3$. Pick $c(y) \in L(y)-\left\{c_{1}(v)\right\}, c(x) \in L(x)-$ $\{c(y)\}$. We replace $L(z)$ by $L(z) \backslash\{c(x)\}$ for every $z \in N(x)-\{x, y\}$ and by $L(z)-\{c(y)\}$ for every $z \in N(y)-\{x, v\}$. Let $G^{\prime}=G-\{y, x\}$ and $A^{\prime}=A \cup N(x) \cup N(y)-\{x, y, v\}$.

If $G[N(x) \cup N(y)]$ does not contain a 4-cycle, by (B2), $x_{6} \notin A$. By Lemma 7.2.5 $G\left[A^{\prime}\right]$ is edgeless and so both $G^{\prime}$ and $A^{\prime}$ satisfy the condition of Theorem 7.2.1. So assume that $G[N(x) \cup N(y)]$ contains a 4-cycle $x q_{1} q_{2} y x$, where $q_{1} \in N(x)$ and $q_{2} \in N(y)$ (possibly $q_{2}=v$ or $q_{1}=x_{i+2}$ ). By Lemma 7.2.5 and (A2), $G^{\prime}\left[A^{\prime}\right]$ contains at most one edge $q_{1} q_{2}$. If $G^{\prime}\left[A^{\prime}\right]$ has that edge $q_{1} q_{2}$, then $q_{2} \neq v$. If there is a 2-path $q_{2} q_{3} q_{4}$ where $q_{4} \in A^{\prime}$, then $q_{3} \neq q_{1}\left(q_{1}=x_{5}\right.$ possibly). By (A2) and (A3), $q_{4} \notin N\left(x_{3}\right) \cup N\left(x_{4}\right)$. Thus $q_{4} \in A$, contrary to Lemma 7.2.6. By (A2) $G\left[\left\{u, v, q_{1}, q_{2}\right\}\right]$ is not a 
4-cycle. Therefore both $G^{\prime}$ and $A^{\prime}$ satisfy the condition of Theorem 7.2.1 with $q_{2}$ playing the role of $x$ of $G$.

By the minimality of $G, c$ can be extended to a list coloring of $G^{\prime}$. Therefore we get a 3-list-cloring of $G$, contrary to (1).

Lemma 7.2.10 If $x_{i-1} x_{i} x_{i+1} x_{i+2} x_{i-1}$ is a facial 4-cycle of $G$, then there is no 3-path $x_{i-1} q_{4} q_{3} x_{i+4}$, where $i \in\{4,5, \ldots, m-4\}$.

Proof. Assume that $P=x_{i-1} q_{4} q_{3} x_{i+4}$ exists. Let $C_{1}$ be the cycle in $C \cup$ $\left\{x_{i-1} q_{4}, q_{4} q_{3}, q_{3} x_{i+4}\right\}$ containing $x_{i-1} q_{4}, q_{4} q_{3}, q_{3} x_{i+4}$ and $u v$ and let $C_{2}$ be the cycle in $C \cup\left\{x_{i-1} q_{4}, q_{4} q_{3}, q_{3} x_{i+4}, x_{i-1} x_{i+2}\right\}$ containing $x_{i-1} q_{4}, q_{4} q_{3}, q_{3} x_{i+4}$ and $x_{i-1} x_{i+2}$ but not $u v$ nor $y x$. Let $G_{i}=C_{i} \cup \operatorname{int}\left(C_{i}\right), i=1,2$.

By the minimality of $G, c$ can be extended to a list coloring of $G_{1}$. We claim that there is not a 2-path from $x_{i+4}$ to $x_{i-1}$ in $G_{2}$. By contradiction, assume that $x_{i+4} z x_{i-1}$ is a 2-path in $G_{2}$. Then we have two 5-cycles $x_{i-1} q_{4} q_{3} x_{i+4} z x_{i-1}$ and $x_{i-1} x_{i+2} x_{i+3} x_{i+4} z x_{i-1}$. By Lemma 7.2.8, $d_{G}(z)=2$, contrary to Lemma 7.2.3.

Pick $c(y) \in L(y)-\left\{c\left(x_{i-1}\right)\right\}, c(x) \in L(x)-\{c(y)\}, c\left(x_{i+2}\right) \in L\left(x_{i+2}\right)-$ $\left\{c\left(x_{i-1}\right), c(x)\right\}$. Let $A^{\prime \prime}=\left\{x_{i+4}, x_{i-1}, x_{i+2}\right\}$. Replace $L\left(x_{i+4}\right)$ by $\left\{c\left(x_{i+4}\right), c\left(q_{3}\right)\right\}$, $L\left(x_{i-1}\right)$ by $\left\{c\left(x_{i-1}\right), c\left(q_{4}\right)\right\}$ and $L\left(x_{i+2}\right)$ by $\left\{c\left(x_{i+2}\right), c\left(x_{i-1}\right)\right\}$. By (A2) $G\left[\left\{q_{3}, q_{4}, x_{i-1}, x_{i+2}\right\}\right]$ is not a 4-cycle. Therefore $G_{2}$ and $A^{\prime \prime}$ satisfy the hypotheses of Theorem 7.2.1 with $x_{i-1}$ playing the role of $x$ of $G$. By the minimality of $G, c$ can be extended to a list coloring of $G_{2}$. Thus we get a list coloring of $G$, contrary to (1).

Lemma 7.2.11 If $G[A]$ has an edge, then $x_{i-2} \in A$ and hence $i \geq 5$. 
Proof. Since $G[A]$ has only one edge, $x_{i-1} \notin A$. By contradiction, suppose that $x_{i-2} \notin A$.

Claim $2 G[N(x) \cup N(y)]$ does not contain a 4-cycle $x y x_{i-1} x_{i+2} x$.

Proof. By contradiction, suppose that $G[N(x) \cup N(y)]$ contains a 4-cycle $x y x_{i-1} x_{i+2} x$. We consider two cases.

Case 1. $x_{i-3} \in A$.

Let $G^{\prime}=G-\left\{x_{i-2}, x_{i-1}, x_{i+2}, x, y\right\}$ and $A^{\prime}=A \cup N\left(x_{i-2}\right) \cup N\left(x_{i-1}\right) \cup$ $N\left(x_{i+2}\right)-\left\{x, y, x_{i-1}, x_{i-2}, x_{i+2}\right\}$. Pick $c\left(x_{i-2}\right) \in L\left(x_{i-2}\right)-L\left(x_{i-3}\right), c\left(x_{i-1}\right) \in$ $L\left(x_{i-1}\right)-\left\{c\left(x_{i-2}\right)\right\}, c(y) \in L(y)-\left\{c\left(x_{i-1}\right)\right\}, c(x) \in L(x)-\{c(y)\}$ and $c\left(x_{i+2}\right) \in$ $L\left(x_{i+2}\right)-\left\{c(x), c\left(x_{i-1}\right\}\right.$. Replace $L(z)$ by $L(z)-\left\{c\left(x_{j}\right)\right.$ if $z \in N\left(x_{j}\right), j \in$ $\{i-1, i-2, i+2\}$. By (A2), (A3) and Lemma 7.2.5, $G^{\prime}\left[A^{\prime}\right]$ contains at most one edge $x_{i+3} x_{i+4}$. If $G^{\prime}\left[A^{\prime}\right]$ contains that edge, then $x_{i+4} \in A$. Assume that there is a 2-path $x_{i+4} q_{3} q_{4}$. By Lemma 7.2.4, $q_{4} \in A^{\prime}-A$. By (A3), $q_{4} \notin N\left(x_{i+2}\right)$. By Lemma 7.2.10, $q_{4} \notin N\left(x_{i-1}\right)$. So assume that $q_{4} \in N\left(x_{i-2}\right)$.

Let $Q=x_{i-2} q_{4} q_{3} x_{i+4}$ and $C_{3}$ be the cycle in $C \cup Q$ containing $u v$ and $C_{4}$ be the cycle in $C \cup Q$ not conaining $u v . G_{i}=C_{i} \cup \operatorname{int}\left(C_{i}\right), i=3,4$. By the minimality of $G, c$ can be extended to a list coloring of $G_{3}$.

Let $A_{2}=A \cap V\left(G_{4}\right) \cup\left\{x_{i-2}, x_{i+4}\right\}$. We replace $L\left(x_{i+4}\right)$ by $\left\{c\left(x_{i+4}\right), c\left(q_{3}\right)\right\}$ and $L\left(x_{i-2}\right)$ by Let $\left\{c\left(x_{i-2}\right), c\left(q_{4}\right)\right\}$.

By Lemma 7.2.4, $G\left[\left\{q_{3}, q_{4}, x, y\right\}\right]$ is not a 4-cycle. Therefore both $G_{4}$ and $A_{2}$ satisfy the hypotheses of Theorem 7.2.1, $c$ can be extended to a list coloring of $G_{4}$. Thus $c$ can be extended to a list coloring of $G$, contrary to (1).

Case $2 x_{i-3} \notin A$. 
Let $G^{\prime}=G-\{x, y\}$ and $A^{\prime}=A \cup\left\{x_{i-1}, x_{i+2}\right\}$. Pick $c(x) \in L(x), c(y) \in$ $L(y)-\{c(x)\}$. Replace $L\left(x_{i-1}\right)$ by $L\left(x_{i-1}\right)-\{c(y)\}$ and $L\left(x_{i+2}\right)$ by $L\left(x_{i+2}\right)-$ $\{c(x)\}$. By (B2), $x_{i-1} x_{i+2}$ is the only edge of $G^{\prime}\left[A^{\prime}\right]$. By Lemma 7.2.5, there is no 2-path from $x_{i-1}$ to a vertex of $A$. By (A2), $G\left[\left\{u, v, x_{i-1}, x_{i+2}\right\}\right]$ is not a 4 cycle. Therefore both $G^{\prime}$ and $A^{\prime}$ satisfy the hypotheses of Theorem 7.2.1 with $x_{i-1}$ playing the role of $x$ of $G$. By the minimality of $G, c$ can be extended to a list coloring of $G^{\prime}$ and hence $c$ can be extended to a list coloring of $G$, contrary to $(1)$.

Now we are ready to complete the proof of Lemma 7.2.11. Pick $c(y) \in$ $L(y), c(x) \in L(y)-\{c(y)\}, G^{\prime}=G-\{x, y\}$ and $A^{\prime}=A \cup N(x) \cup N(y)-\{x, y\}$. By $(\mathrm{B} 2), x_{i+3} \notin A^{\prime}$.

If $G[N(x) \cup N(y)]$ does not contain a 4-cycle, then by Lemma 7.2.5 and (B2), $G\left[A^{\prime}\right]$ is edgeless. So assume that $G[N(x) \cup N(y)]$ contains a 4-cycle $x q_{1} q_{2} y x$, where $q_{1} \in N(x)$ and $q_{2} \in N(y)$. By Lemma 7.2.5 and (A2), $G^{\prime}\left[A^{\prime}\right]$ contains only one edge $q_{1} q_{2}$ ( possibly $q_{1}=x_{i+2}$ or $q_{2}=x_{i-1}$ ). By Claim 2 , we assume that $q_{2} \neq x_{i-1}$. Suppose that there is a 2-path $q_{2} q_{3} q_{4}$, where $q_{4} \in A^{\prime}$. Since $G^{\prime}\left[A^{\prime}\right]$ has only one edge, $q_{3} \neq q_{1}$. By (A2) and (A3), $q_{4} \in$ $A-N(x) \cup N(y)$, contrary to Lemma 7.2.6. By (A2), $G^{\prime}\left[\left\{q_{1}, q_{2}, u, v\right\}\right]$ is not a 4-cycle. Therefore both $G^{\prime}$ and $A^{\prime}$ satisfy the hypotheses of Theorem 7.2.1 with $q_{2}$ playing the role of $x$ of $G$.

Replace $L(z)$ by $L(z)-\{c(x)\}$ if $z \in N(x)-A$ and by $L(z)-\{c(y)\}$ if $z \in N(y)-A$. By the minimality of $G, c$ can be extended to a list coloring of $G^{\prime}$. Then $c$ can be extended to a list coloring of $G$, contrary to (1). The proof for the case when $q_{1} \neq x_{i+2}$ is similar. 
Lemma 7.2.12 $G[W]$ has no edge.

Proof. Suppose that $G[A]$ has the edge $x y$ where $x=x_{i+1}, y=x_{i}$. By Lemma 7.2.11 and by (B2), $x_{i-1} \notin A$ and $x_{i-2} \in A$.

Case $1 G[N(x) \cup N(y)]$ contains no a 4-cycle.

Let $G^{\prime}=G-\left\{x_{i-1}, x_{i}, x_{i+1}\right\}$ and $A^{\prime}=A \cup N\left(x_{i-1}\right) \cup N\left(x_{i}\right) \cup N\left(x_{i+1}\right)-$ $\left\{x_{i-1}, x_{i}, x_{i+1}\right\}$. Let $c\left(x_{i-1}\right) \in L\left(x_{i-1}\right)-\left\{c\left(x_{i-2}\right)\right\}, c\left(x_{i}\right) \in L\left(x_{i}\right)-\left\{c\left(x_{i-1}\right)\right\}$ and $c\left(x_{i+1}\right) \in L\left(x_{i+1}\right)-\left\{c\left(x_{i}\right)\right\}$. Replace $L(z)$ by $L(z)-\left\{c\left(x_{j}\right)\right\}$ for every vertex $z \in\left(A^{\prime}-A\right) \cap\left(N\left(x_{i-1}\right) \cup N\left(x_{i}\right) \cup N\left(x_{i+1}\right)\right)$. Suppose that $G^{\prime}\left[A^{\prime}\right]$ has an edge $q_{1} q_{2}$ where $q_{1}, q_{2} \in A^{\prime}-A \subset N\left(x_{i-1}\right) \cup N\left(x_{i}\right) \cup N\left(x_{i+1}\right)$. We will distinguish the following two subcases.

Subcase $1.1 q_{2} \in N\left(x_{i-1}\right), q_{1} \in N\left(x_{i+1}\right)$.

Then $G$ has a 5 -cycle $q_{1} q_{2} x_{i-1} x_{i} x_{i+1} q_{1}$. Note that $q_{1}=x_{i+2}$ is possible. By Lemmas 7.2.3 and 7.2.8 $q_{2} \neq x_{i-2}$. By (A2), (A3), (B2) and Lemma 7.2.5, $q_{1} q_{2}$ is the only edge in $G^{\prime}\left[A^{\prime}\right]$.

Assume that $G^{\prime}$ has a path $q_{1} q_{3} q_{4}$ where $q_{4} \in A^{\prime}$. As $G^{\prime}\left[A^{\prime}\right]$ has only one edge, $q_{3} \neq q_{2}$.

We claim that $q_{4} \in A$. By contradiction, suppose $q_{4} \in A^{\prime}-A$. If $q_{4} \in$ $N\left(x_{i-1}\right)-q_{2}$, then $G$ has two 5 -cycles $x_{i-1} x_{i} x_{i+1} q_{1} q_{2} x_{i-1}$ and $x_{i-1} q_{4} q_{3} q_{1} q_{2} x_{i-1}$. By Lemma 7.2.8, $d_{G}\left(q_{2}\right)=2$, contrary to Lemma 7.2.3. Since $G$ has a 5-cycle $q_{1} q_{2} x_{i+1} x_{i} x_{i-1} q_{1}, q_{4} \notin N\left(x_{i}\right)$. By (A3), $q_{4} \notin N\left(x_{i+1}\right)$. Thus $q_{4} \in A$.

By Lemmas 7.2.4, 7.2.5 and 7.2.6, $q_{1}=x_{i+2}, q_{3}=x_{i+3}$ and $q_{4}=x_{i+4}$. It follows that $d_{G}\left(x_{i}\right)=d_{G}\left(x_{i+1}\right)=2$. Note that $q_{2} \neq x_{i-2}$.

We shall verify that both $G^{\prime}$ and $A^{\prime}$ satisfy the other hypotheses of Theorem 7.2.1 with $q_{1}$ and $q_{2}$ replacing $y$ and $x$, respectively. 
We now assume that $G^{\prime}$ has a path $q_{2} q_{5} q_{6}$ where $q_{6} \in W^{\prime}$. By (A3), $q_{6} \notin$ $A^{\prime}-A$ and hence $q_{6} \in A$. If $q_{5} \notin V(C)$, then it contradicts to Lemma 7.2.7(i). If $q_{5} \in V(C)$, then it contradicts to Lemma 7.2.7(ii). Thus there is no 2-path from $q_{2}$ to a vertex of $A^{\prime}$ in $G^{\prime}$.

By (A2) $G^{\prime}\left[\left\{u, v, q_{1}, q_{2}\right\}\right]$ is not a 4-cycle. Therefore both $G^{\prime}$ and $A^{\prime}$ satisfy the hypotheses of Theorem 7.2.1 with $q_{2}$ playing the role of $x$ of $G$. Thus $c$ can be extended to a list coloring of $G^{\prime}$. Therefore $c$ is a list coloring of $G$, contrary to (1).

Subcase $1.2 q_{2} \in N\left(x_{i-1}\right), q_{1} \in N\left(x_{i}\right)$.

If $q_{2}=x_{i-2}$, then $d_{G}\left(x_{i-1}\right)=2$, contrary to Lemma 7.2.3. So $q_{2} \in$ $V(G)-V(C)$. Since $G[N(x) \cup N(y)]$ does not contain a 4-cycle, $q_{1} \neq x_{i+1}$. So we assume that $q_{2} \neq x_{i-2}$ and $q_{1} \neq x_{i+1}$. If there is a 2-path $q_{1} q_{3} q_{4}$, where $q_{4} \in A^{\prime}$, by (A3) and by Lemma 7.2.8 $q_{4} \notin N\left(x_{i+1}\right) \cup N\left(x_{i}\right) \cup N\left(x_{i-1}\right)$. Thus $q_{4} \in A$, contrary to Lemma 7.2.6. By (A2) $G^{\prime}\left[\left\{u, v, q_{1}, q_{2}\right\}\right]$ is not a 4-cycle.

Therefore both $G^{\prime}$ and $A^{\prime}$ satisfy the condition of Theorem 7.2.1 with $q_{1}$ playing the role of $x$ of $G$. Thus $c$ can be extended to a list coloring of $G^{\prime}$. Therefore $c$ can be extended to a list coloring of $G$, contrary to (1).

Case $2 G[N(x) \cup N(y)]$ contains a 4-cycle.

Without loss of generality, we assume that $x q_{1} q_{2} y x$ is a 4-cycle in $G[N(x) \cup$ $N(y)$ ], where $q_{1} \in N\left(x_{i+1}\right), q_{2} \in N\left(x_{i}\right)$. We distinguish the following two subcases.

Subcase $2.1 q_{2} \neq x_{i-1}$.

Let $G^{\prime}=G-\left\{x_{i-1}, x_{i}, x_{i+1}\right\}$ and $A^{\prime}=A \cup N\left(x_{i-1}\right) \cup N\left(x_{i}\right) \cup N\left(x_{i+1}\right)$ $\left\{x_{i-1}, x_{i}, x_{i+1}\right\}$. Let $c\left(x_{i-1}\right) \in L\left(x_{i-1}\right)-L\left(x_{i-2}\right), c\left(x_{i}\right) \in L\left(x_{i}\right)-\left\{c\left(x_{i-1}\right)\right\}$ 
and $c\left(x_{i+1}\right) \in L\left(x_{i+1}\right)-\left\{c\left(x_{i}\right)\right\}$. Replace $L(z)$ by $L(z)-\left\{c\left(x_{j}\right)\right.$ for every vertex $z \in\left(A^{\prime}-A\right) \cap\left(N\left(x_{i-1}\right) \cup N\left(x_{i}\right) \cup N\left(x_{i+1}\right)\right)$.

Claim $2 G^{\prime}\left[A^{\prime}\right]$ has only one edge.

Note that $q_{1} q_{2} \in G^{\prime}\left[A^{\prime}\right]$. Suppose that there is another edge $p_{1} p_{2}$ of $G^{\prime}\left[A^{\prime}\right]$. By (B2) $x_{i+3} \notin A$. It follows that $x_{i+2} x_{i+3} \notin E\left(G^{\prime}\left[A^{\prime}\right]\right)$. By Lemmas 7.2.4 and 7.2.5, we may assume that either $p_{2} \in N\left(x_{i-1}\right), p_{1} \in N\left(x_{i+1}\right)$, or $p_{2} \in$ $N\left(x_{i-1}\right), p_{1} \in N\left(x_{i}\right)$, or $p_{2} \in N\left(x_{i}\right), p_{1} \in N\left(x_{i+1}\right)$. Each case contradicts (A2) or (A3).

Claim 3 There is no 2-path from $q_{2}$ to every vertex of $A^{\prime}$.

By contradiction, suppose that $G^{\prime}$ has a path $q_{2} q_{3} q_{4}$ where $q_{4} \in A^{\prime}$. As $G^{\prime}\left[A^{\prime}\right]$ has only one edge,$q_{3} \neq q_{1}$. By (A2) and (A3), $q_{4} \notin N\left(x_{i-1}\right) \cup N\left(x_{i}\right) \cup$ $N\left(x_{i+1}\right)$. So $q_{4} \in A$, contrary to Lemma 7.2.6. So both $G^{\prime}$ and $A^{\prime}$ satisfy the condition of Theorem 7.2.1 with $q_{2}$ playing the role of $x$ of $G$. By the choice of $G, c$ can be extended to a list coloring of $G^{\prime}$ and hence $c$ can be extended to a list coloring of $G$, contrary to $(1)$.

Subcase $2.2 q_{2}=x_{i-1}$.

Let $G^{\prime}=G-\left\{x_{i-1}, y, x, q_{1}\right\}$ and $A^{\prime}=A \cup N\left(x_{i-1}\right) \cup N(x) \cup N\left(q_{1}\right)-$ $\left\{x_{i-1}, x, y, q_{1}\right\}$.

Pick $c\left(x_{i-1}\right) \in L\left(x_{i-1}\right)-L\left(x_{i-2}\right), c(y) \in L(y)-\left\{c\left(x_{i-1}\right)\right\}, c(x) \in L(x)-$ $\{c(y)\}, c\left(q_{1}\right) \in L\left(q_{1}\right)-\left\{c\left(x_{i-1}\right), c(x)\right\}$.

Replace $L(z)$ by $L(z)-c(\lambda)$ where $\lambda \in\left\{x_{i-1}, q_{1}, x\right\}, z \in N\left(x_{i-1}\right) \cup N\left(q_{1}\right) \cup$ $N(x)-A$.

By (A2), (A3) and Lemma $7.2 .8, G^{\prime}\left[A^{\prime}\right]$ has at most one edge $x_{i+3} x_{i+4}$. If $G^{\prime}\left[A^{\prime}\right]$ contains that edge, then $x_{i+4} \in A, x_{i+2}=q_{1}$. If there is a 2-path 
$x_{i+4} q_{5} q_{6}$, where $q_{6} \in A^{\prime}$. By Lemma 7.2.6, $q_{6} \in A^{\prime}-A$. By (A3), $q_{6} \in N\left(q_{2}\right)$, contrary to Lemma 7.2.10. By Lemma 7.2.3 $G^{\prime}\left[\left\{x_{i+3}, x_{i+4}, u, v\right\}\right]$ is not a 4cycle. Therefore both $G^{\prime}$ and $A^{\prime}$ satisfy the hypotheses of Theorem 7.2.1 with $x_{i+4}$ playing the role of $x$ of $G$. By the minimality of $G, c$ can be extended to a list coloring of $G^{\prime}$ amd hence $c$ can be extended to a list coloring of $G$, contrary to (1).

Lemma 7.2.13 $x_{4} \in A$.

Proof. Suppose that $x_{4} \notin A$. Let $G^{\prime}=G-x_{3}$ and $A^{\prime}=A \cup N\left(x_{3}\right)-$ $\left\{x_{2}, x_{3}\right\}$. Pick $c\left(x_{3}\right) \in L\left(x_{3}\right)-\left\{c\left(x_{2}\right)\right\}$. Replace $L(z)$ by $L(z)-\left\{c\left(x_{3}\right)\right\}$ if $z \in N\left(x_{3}\right)-x_{2}$.

By Lemmas 7.2.4, 7.2.5 and 7.2.12, $G^{\prime}\left[A^{\prime}\right]$ has at most one edge, namely $x_{4} x_{5}$. If $G^{\prime}\left[A^{\prime}\right]$ contains $x_{4} x_{5}$, then $x_{5} \in A$. If there is a 2-path $x_{5} q_{5} q_{6}$, where $q_{6} \in A^{\prime}-A$, then $q_{6} \in N\left(x_{3}\right)-\left\{x_{4}\right\}, q_{5} \neq x_{4}$. $G$ has a 5-cycle $x_{3} x_{4} x_{5} q_{5} q_{6} x_{3}$. By Lemma 7.2.8, $d_{G}\left(x_{4}\right)=2$, contrary to Lemma 7.2.3. By (A1), $G\left[\left\{u, v, x_{4}, x_{5}\right\}\right]$ does not contain a 4-cycle.

So both $G^{\prime}$ and $A^{\prime}$ satisfy the hypotheses of Theorem 7.2 .1 with $x_{5}$ playing the role of $x$ of $G$. By the minimality of $G, c$ can be extended to a list coloring of $G$, contrary to (1).

\section{Lemma 7.2.14 $|C| \geq 6$.}

Proof. Suppose that $|C|=5$. Let $G^{\prime}=G-\left\{x_{3}, x_{4}\right\}$ and $A^{\prime}=A \cup N\left(x_{3}\right) \cup$ $N\left(x_{4}\right)-\left\{x_{2}, x_{3}, x_{4}\right\}$. Let $c\left(x_{3}\right) \in L\left(x_{3}\right)-\left\{c\left(x_{2}\right)\right\}$ and $c\left(x_{4}\right) \in L\left(x_{4}\right)-\left\{c\left(x_{3}\right)\right\}$. Replace $L(z)$ by $L(z)-c\left(x_{i}\right)$ where $z \in N\left(x_{i}\right)-\left\{x_{2}, x_{3}, x_{4}\right\}, i=3$, 4. Assume first that $G\left[N\left(x_{3}\right) \cup N\left(x_{4}\right)\right]$ does not contain a 4-cycle. It follows that $G^{\prime}\left[A^{\prime}\right]$ 
is edgeless. So assume that $G\left[N\left(x_{3}\right) \cup N\left(x_{4}\right)\right]$ contains a 4-cycle and assume that $x_{3} q_{2} q_{1} x_{4} x_{3}$ is a 4-cycle, where $q_{1} \in N\left(x_{4}\right), q_{2} \in N\left(x_{3}\right)$. By (A2) and Lemma $7.2 .5, G^{\prime}\left[A^{\prime}\right]$ has only one edge $q_{1} q_{2}$. If $q_{2}=x_{2}$, by $(\mathrm{A} 1), d_{G}\left(x_{3}\right)=2$, contrary to Lemma 7.2.3. So assume that $q_{2} \neq x_{2}$. Assume that $q_{1}=x_{5}$. If $G^{\prime}\left[A^{\prime}\right]$ has an edge $e$, by (A1) $e=x_{5} v^{\prime}$, where $v^{\prime} \in N\left(x_{3}\right)$. Thus $G$ has a 5-cycle $x_{1} x_{2} x_{3} v^{\prime} x_{5} x_{1}$ and a 4-cycle $x_{3} x_{4} x_{5} v^{\prime} x_{3}$. By Lemma 7.2.8 and (A1), $d_{G}\left(v^{\prime}\right)=2$, comtrary to Lemma 7.2.3.

Now assume that $q_{1} \neq x_{5}$ and $q_{2} \neq x_{2}$. By (A2) and (A3), there is no 2-path from $q_{2}$ to a vertex of $A^{\prime}$. So both $G^{\prime}$ and $A^{\prime}$ satisfy the hypotheses of Theorem 7.2.1 with $q_{2}$ playing the role of $x$ of $G$. By the minimality of $G$, $c$ can be extended to a list coloring of $G^{\prime}$ and hence $c$ can be extended to a list coloring of $G$, contrary to (1).

Lemma 7.2.15 $x_{6} \in A$.

Proof. Suppose that $x_{6} \notin A$. We will distinguish the following two cases.

Case $1 G\left[N\left(x_{3}\right) \cup N\left(x_{4}\right)\right]$ does not contain a 4-cycle.

Let $c\left(x_{3}\right) \in L\left(x_{3}\right)-\left\{c\left(x_{2}\right)\right\}, c\left(x_{4}\right) \in L\left(x_{4}\right)-\left\{c\left(x_{3}\right)\right\}$. Put $G^{\prime}=G-$ $\left\{x_{3}, x_{4}\right\}$ and $A^{\prime}=A \cup N\left(x_{3}\right) \cup N\left(x_{4}\right)-\left\{x_{2}, x_{3}, x_{4}\right\}$. Since $G\left[N\left(x_{3}\right) \cup N\left(x_{4}\right)\right]$ does not contain a 4-cycle, by Lemma $7.2 .5 G^{\prime}\left[A^{\prime}\right]$ is edgeless. Replace $L(z)$ by $L(z)-\left\{c\left(x_{j}\right)\right\}$ if $z \in\left(A^{\prime}-A\right) \cap\left(N\left(x_{3}\right) \cup N\left(x_{4}\right)\right)-\left\{x_{5}\right\}$.

By the choice of $G, c$ can be extended to a list coloring of $G^{\prime}$ hence $c$ can be extended to a list coloring of $G$, violating to (1).

Case $2 G\left[N\left(x_{3}\right) \cup N\left(x_{4}\right)\right]$ contains a 4-cycle.

Let $c\left(x_{4}\right) \in L\left(x_{4}\right), c\left(x_{5}\right) \in L\left(x_{5}\right)-\left\{c\left(x_{4}\right)\right\}$. Put $G^{\prime}=G-\left\{x_{4}, x_{5}\right\}$ and 
$A^{\prime}=A \cup N\left(x_{4}\right) \cup N\left(x_{5}\right)-\left\{x_{4}, x_{5}\right\}$. By (B2), $G\left[N\left(x_{4}\right) \cup N\left(x_{5}\right)\right]$ does not contains a 4-cycle.

If $x_{7} \notin A$, by (A2), $G^{\prime}\left[A^{\prime}\right]$ is edgeless. Assume that $x_{7} \in A$. By (A2) and Lemma $7.2 .4, G^{\prime}\left[A^{\prime}\right]$ has only one edge $x_{6} x_{7}$. If $G^{\prime}$ has a 2-path $x_{7} q_{5} q_{6}$, where $q_{6} \in A^{\prime}$, then by Lemma 7.2.5, $q_{6} \in N\left(x_{4}\right) \cup N\left(x_{5}\right)$. By (A3) and (A1), $q_{5} \neq x_{6}$. If $q_{6} \in N\left(x_{5}\right)$, then $G$ has a 5 -cycle $x_{5} q_{6} q_{5} x_{7} x_{6} x_{5}$. By Lemma 7.2.8 $d_{G}\left(x_{6}\right)=2$, contrary to Lemma 7.2.3. By Lemma 7.2.5, $q_{6} \notin N\left(x_{4}\right)$. By Lemma 7.2.4, $G^{\prime}\left[\left\{u, v, x_{6}, x_{7}\right\}\right]$ is not a 4-cycle.

Therefore both $G^{\prime}$ and $A^{\prime}$ satisfy the condition of Theorem 7.2.1 with $x_{7}$ playing the role of $x$ of $G$. By the minimality of $G, c$ can be extended to a list coloring of $G^{\prime}$ and hence $c$ is a list coloring of $G$, violating to (1).

Proof of Theorem 7.2.1. Let $\left.c\left(x_{5}\right) \in L\left(x_{5}\right)-L 9 x_{6}\right), c\left(x_{4}\right) \in L\left(x_{4}\right)-$ $\left\{c\left(x_{5}\right)\right\}$. Put $G^{\prime}=G-\left\{x_{4}, x_{5}\right\}$ and $A^{\prime}=A \cup N\left(x_{4}\right) \cup N\left(x_{5}\right)-\left\{x_{4}, x_{5}\right\}$. Replace $L(z)$ by $L(z)-\left\{c\left(x_{j}\right)\right\}$ if $z \in N\left(x_{j}\right), 3 \leq j \leq 5$.

If $G\left[N\left(x_{4}\right) \cup N\left(x_{5}\right)\right]$ does not contain a 4-cycle, by Lemma 7.2.5 $G^{\prime}\left[A^{\prime}\right]$ is edgeless. Assume that $G\left[N\left(x_{4}\right) \cup N\left(x_{5}\right)\right]$ contains a 4-cycle. By (A2) and Lemma 7.2.4, $G^{\prime}\left[A^{\prime}\right]$ contains only edge $q_{1} q_{2}$, where $q_{2} \in N\left(x_{4}\right)$ and $q_{1} \in N\left(x_{5}\right)$. By Lemma 7.2.3, $q_{1} \neq x_{6}$. Let $q_{2} q_{3} q_{4}$ be a 2-path where $q_{4} \in A^{\prime}$. By (A2) and (A3), $q_{4} \in A$, contrary to Lemma 7.2.6. Therefore both $G^{\prime}$ and $A^{\prime}$ satisfy the condition of Theorem 7.2 .1 with $q_{2}$ playing the role of $x$ of $G$.

By the minimality of $G, c$ can be extended to a list coloring of $G^{\prime}$ and hence $c$ can be extended to a list coloring of $G$, contrary to (1).

The proof of Theorem 7.2.1 is complete. 


\subsection{3-list coloring of $K_{3,3}$-minor free graphs}

Let $G_{1}$ and $G_{2}$ be two bridgeless graphs and $u_{1}, v_{1} \in V\left(G_{1}\right), u_{2}, v_{2} \in V\left(G_{2}\right)$. We define $G$ as follows: identify $u_{1}$ with $u_{2}$ and $v_{1}$ with $v_{2}$. We define these two new vertices $u$ and $v$. Then $G$ is called 2-sum of $G_{1}$ and $G_{2}$. Similarly let $u \in V\left(G_{1}\right)$ and $v \in V\left(G_{2}\right)$. G is obtained from $G_{1}$ and $G_{2}$ by identifying $u$ with $v$ and $G$ is called 1 -sum of $G_{1}$ and $G_{2}$.

Theorem 7.3.1 (Hall [28]) Let $G$ be a graph without $K_{3,3}$ minor. One of the followings must holds.

(1) $G$ is a planar graph;

(2) $G \cong K_{5}$ and

(3) for some $i \in\{1,2\}, G$ is the $i$-sum of two $G_{1}$ and $G_{2}$ such that $G_{1}$ and $G_{2}$ are proper minor of $G$.

Corollary 7.3.2 Suppose that $G$ be a simple planar graph and $H=K_{2}=$ $G[\{u, v\}]$. Let $c(u)$ and $c(w)$ be distinct colors in $L(u)$ and $L(w)$ respectively. Then c can be extended to a list coloring of $G$.

Proof. We assume that $u v$ is on the exterior cycle of $G$ in an planar embedding of $G$. By Theorem 7.3.1, $c$ can be extended to a list coloring of $G$.

Theorem 7.3.3 Suppose that $G$ is a connected $K_{3,3}$-minor free graph with girth $\geq 5$ and that $|L(v)| \geq 3$ for each vertex of $G$. Then there is a subgraph $H=K_{2}=G[\{u, v\}]$ of $G$ such that two different colors of $c(u) \in L(u)$ and $c(v) \in L(v)$ can be extended to a list coloring of $G$. 
Proof. The proof is by contradiction. Assume that $G$ is a counterexample with $|V(G)|$ minimum. We claim that $G$ is 2-connected. By contradiction, suppose that $G$ is not 2-connected, then there is a block $B$ such that $G-V(B)$ is connected. We color $B$ at first, then coloring each block having a vertex in common with $B$ and so on. We finally get a list coloring of $G$, a contradiction.

By Theorem 7.3.1, $G$ is a 2 -sum of $G_{1}$ and $G_{2}$ which are proper minor of $G$ and assume that $G_{1}$ is planar with $\left|V\left(G_{1}\right)\right|$ minimized. Since $G$ has girth $\geq 5$, we may assume that $|V(G)| \geq 5$.

Let $V\left(G_{1}\right) \cap V\left(G_{2}\right)=\{u, v\}$. If $u v \in E(G)$, let $c(u)$ and $c(v)$ be distinct colors in $L(u)$ and $L(v)$ respectively. By the minimality of $G, c$ can be extended to a list coloring of $G_{2}$. By Corollary 7.3.2, $c$ can be extended to a list coloring of $G_{1}$. So $c$ can be extended to a list coloring of $G$, a contradiction.

So we assume that $u v \notin E(G)$. We claim that $G_{1}+u v$ is also planar. By contradiction, we assume that $G_{1}+u v$ is not planar. If $G_{1}+u v$ has a $K_{3,3^{-}}$ minor $\Gamma$, then new edge $u v \in E(\Gamma)$ since $G_{1}$ does not have a $K_{3,3}$-minor. Since $G$ is 2-connected and since $\{u, v\}$ is 2-vertex cut of $G, G_{2}$ must have a $(u, v)$ path. It then follows that $G$ has a $K_{3,3}$-minor, contrary to the assumption of $G$. Therefore $G_{1}+u v$ does not have a $K_{3,3}$-minor. By Theorem 7.3.1, $G_{1}+u v \cong K_{5}$. contrary to the assumption that $G$ has girth at least 5 . Thus $G_{1}+u v$ must be planar. Define $G^{*}$ obtained from $G$ by adding two new vertices $v_{1}, v_{2}$ and three new edges $u v_{1}, v_{1} v_{2}, v_{2} v$ such that $G_{i}^{*}$ is obtained from $G_{i}$ by adding these two vertices and these three edges $(1 \leq i \leq 2)$. It follows that $G_{1}^{*}$ is planar and $G^{*}, G_{1}^{*}$ and $G_{2}^{*}$ have girth $\geq 5$. Define $L\left(v_{2}\right)=$ $L(u), L\left(v_{1}\right)=L(v)$. Then for every vertex $z \in V\left(G^{*}\right),|L(z)| \geq 3$. Let $c\left(v_{1}\right)$ 
and $c\left(v_{2}\right)$ be distinct in $L\left(v_{1}\right)$ and $L\left(v_{2}\right)$ respectively. By the minimality of $G, c$ can be extended to a list coloring of $G_{2}^{*}$.

Now we re imbed $G_{1}^{*}$ in the plane such that the edges $u v_{1}, v_{1} v_{2}, v_{2} v$ are in the exterior cycle of $G_{1}^{*}$. Let $A=\{u, v\}$ and replace $L(u)$ by $\left\{c(u), c\left(v_{1}\right)\right\}$ and $L(v)$ by $\left\{c(v), c\left(v_{2}\right)\right\}$. By Theorem 7.2.1, the colors of $v_{1}$ and $v_{2}$ can be extended to a list coloring $c$ of $G_{1}^{*}$. Thus $G$ is 3-choosable. 


\section{Bibliography}

[1] R.E.L. Aldred, S. Bau, D.A. Holton, and Brendan D. McKay, Cycles Through 23 Vertices in 3-Connected Cubic Planar Graphs. Graphs and Combinatorics (1999) 15: 373-376.

[2] N. Alon and M. Tarsi, Colorings and orientations of graphs, Combinatorica 12(1992), 125-134.

[3] K. Ando, H. Enomoto and A. Saito, Contractible Edges in 3-connected Graphs, J. combinatorial Theory, Ser B 42(1987)87-93.

[4] D. W. Barnette, Cycle covers of planar 3-connected graphs, J. of Combin. Math and Combin. Computing, 20 (1996), 245-253.

[5] S. Bau and D. Holton, Cycles containing 12 vertices in 3-connected cubic graphs. J. Graph Theory, 15 (1991), 421-429.

[6] A. Benhocine, L. Clark, N. Köhler and H. J. Veldman, On circuits and pancyclic line graphs, J. Graph Theory 10 (1986), 411 - 425.

[7] J.A. Bondy, Small cycle double covers of graphs, 'Cycles and Rays', Kluwer Academic Publishers, Dordecht, 1990, 21-40. 
[8] J. A. Bondy and U. S. R. Murty, Graph Theory with Applications, American Elsevier, New York, (1976).

[9] H. J. Broersma, M. Kriesell and Z. Ryjacek, On connected factors in 4-connected claw-free graphs, preprint.

[10] R. L. Brooks, On coloring the nodes of a network, Proc. Cambridge Phil. Soc. 37(1941)194-197.

[11] P. A. Catlin, Supereulerian graphs: A survey, J. Graph Theory, 16 (1992) 177-196.

[12] P. A. Catlin, A reduction method to find spanning Eulerian subgraphs, J. Graph Theory, 12 (1988) 29-44.

[13] P. A. Catlin and Hong-Jian Lai, Spanning trails joining two given edges, "Graph theory, conbinatorics, and applications", eds. by Y. Alavi, G. Chartrand, O. R. Ollermann, A. J. Schwenk, John Wiley and Sons, Inc. (1991) 207-222.

[14] P. A. Catlin, Edge-connectivity and edge-disjoint spaning trees, Ars Combinatoria, accepted.

[15] P. A. Catlin, Z. Han and H.-J. Lai, Graphs without spanning closed trails, Discrete Math. 160 (1996) 81-91.

[16] Z. H. Chen and H.-J. Lai, Reduction techniques for supereulerian graphs and related topics, An update, "Combinatorics and Graphs Theory'95", (ed. Ku Tung-Hsin), World Scientific, Singapore, (1995) 53-69. 
[17] Z.-H. Chen and H.-J. Lai, Collapsible graphs and matchings. J. Graph Theory, 17 (1993), 567 - 605.

[18] Z.-H. Chen and H.-J. Lai, Supereulerian graphs and the Petersen graph, II., Ars Combinatoria, 48 (1998), 271-282.

[19] Z.-H. Chen, H.-J. Lai, H. Y. Lai and G. Q. Weng, Jackson's conjecture on eulerian subgraphs, Combinatorics, Graph Theory, Algorithms and Applications, (eds. by Y. Alavi et al), 53-58, World Scientific, River Edge, NJ, (1994).

[20] Z.-H. Chen, H.-J. Lai, X. Zhang, and L. Xu, Group coloring and group connectivity of graphs, Congressus Numerantium, 134 (1998), 123-130.

[21] N. Dean, R. L. Hemminger and K. Ota, Longest Cycles in 3- Connected Graphs Contain Three contractibles edges, J. Graph Theory 13(1989) $17-21$.

[22] P. Erdos, A. L. Rubin and H. Taylor, Choosability in graphs, Proceedings of the West Coast Conference on Combinatorics, Graph Theory and Computing, Arcata, California, 5.-7.9.1979, Congressus Numeratium XXVI.

[23] G. Fan, Subgraph covering and edge switchings, J. Combin. Theory, Ser. B, to appear.

[24] H. Fleischner, Eulerian Graphs and Related Topics, Part 1, Vol. 1, Ann. Discrete Math., 45 (North Holland, Amsterdam, 1990). 
[25] J. L. Gross and T. W. Tucker, Topological Graph Theory, John Willey \& Sons (1987).

[26] S. Gutner, The complexity of planar graph choosability, Discrete Math. 159(1996) 119-130.

[27] R. Halin, Untersuchungen Uber Minimale n-fach zusammenhaagende Graphen, Math.ann 182(1969)175-188.

[28] D. W. Hall, A note on primitive skew curves. Bull. Amer. Math. Soc. 49(1943)932-937.

[29] F. Harary and C. St. J. A. Nash-Williams, On eulerian and hamiltonian graphs and line graphs, Canad. Math. Bull. 8 (1965), 701-710.

[30] A. J. Hoffman and R. R. Singleton, On Moore graphs with diameters 2 nad 3. IBM J. Res. Development 4(1960)497-504.

[31] D.A. Holton, Cycles in 3-connectd cubic planar graphs, Ann. Discrete Math. 27, 219-226 (1985).

[32] D.A. Holton and J. Sheehan, "The Petersen Graph". Cambridge University Press (1993)

[33] F. Jaeger, Nowhere-zero flow problems, in "Seleted Topics in Graph Theory" (L.Beineke and R. Wilson, Eds.), Vol. 3, pp. 91-95, Acdemic Press, London/New York, 1988.

[34] F. Jaeger, N. Linial, C. Payan, and M. Tarsi, Graph Connectivity of Graphs-A Nonhomogeneous Analogue of Nowhere-Zero Flow Properties, J. Combin. Theory Series B 56, 165-182(1992). 
[35] F. Jaeger, A note on subeulerian graphs, J. Graph Theory, 3 (1979), 91-93.

[36] Jensen and Toft, "Graph Coloring Problems", pp210-211, Wiley and Sons, New York, 1995.

[37] M. Kriesell, All 4-connected line graphs of claw free graphs are hamiltonian connected, J. Combinatorial Theory, Ser. B., to appear.

[38] Hong-Jian Lai and Xiankun Zhang, Group Colorability of Graphs, Ars Combinatorics, accepted.

[39] Hong-Jian Lai and Xiankun Zhang, Group Colorability of Graphs without $K_{5}$-minors, Graphs and Combinatorics, accepted.

[40] H.-J. Lai, Eulerian subgraphs containing given vertices and Hamiltonian line graphs, Discrete Math. 178 (1998), 93-107.

[41] Y. X. Luo and R. S. Chen, Cycle covers of 2-connected 3-regular graphs, (Chinese, Chinese summary), Math. Appl. 9 (1996), suppl., 23-25.

[42] W. Mader, A reduction method for edge-connectivity in graphs, Advances in Graph Theory, Ann. Discrete Math., 3 (North Holland, Amsterdam, 1978).

[43] C. St. J. A. Nash-Williams, Edge-disjoint spanning trees of finite graphs, J. London Math Soc. 36 (1961) 445-450.

[44] W. R. Pulleyblank, A note on graphs spanned by Eulerian graphs, J. Graph Theory, 3 (1979), 309-310. 
[45] P. D. Seymour, Sums of circuits, in: J. A. Bondy and U. S. R. Murty, eds., Graph Theory and related Topics, Academic Press, New York, (1979), 341-355.

[46] G. Szekeres, Polyhedral decomposition of cubic graphs, Bull. Austral. Math. Soc., 8, (1973), 367-387.

[47] C. Thomassen, Reflections on graph theory, J. Graph Theory, 10 (1986) $309-324$.

[48] C. Thomassen, Every planar graph is 5-choosable, J. Combin. Theory Ser. B 62(1994), 180-181.

[49] C. Thomassen, 3-list-coloring planar graphs of girth 5, J. Combin. Theory Ser. B 64(1995), 101-107.

[50] W. T. Tutte, On the problem of decomposing a graph into $n$ connected factors, J. London Math Soc. 36 (1961) 80-91.

[51] W.T.Tutte, A Theory of 3-connected graphs, indag. math 23(1961)441445.

[52] H. J. Veldman, On dominating and spanning circuits in graphs. Discrete Math., 124 (1994), 229 - 239.

[53] V. G. Vizing, coloring the vertices of a graph in prescribed colors (Russian), Diskret. Analiz No 29 Metody Diskret. anal. v Teorii Kodov i Shem(1976), 3-10, 101(MR58\#16371).

[54] M. Voigt, List colorings of planar graphs, Discrete Math. 120(1993) 215219. 
[55] K. Wagner, Über eine eigneschaft der ebenen komplexe. math. Ann. 144 (1937), 570-590.

[56] S. Zhan, Hamiltonian connectedness of line graphs. Ars Combinatoria, 22 (1986) 89-95.

[57] S. Zhan, On hamiltonian line graphs and connectivity. Discrete Math., 89 (1991) $89-95$.

[58] C. Q. Zhang, Integer Flows and Cycle Covers of Graphs. Marcel Dekker, New York, 1997. 